\title{
Kilonova Luminosity Function Constraints Based on Zwicky Transient Facility Searches for 13 Neutron Star Merger Triggers during 03
}

Mansi M. Kasliwal ${ }^{1}$ (D), Shreya Anand ${ }^{1}$ (D), Tomás Ahumada ${ }^{2}$ (D), Robert Stein ${ }^{3,4}$ (iD), Ana Sagués Carracedo ${ }^{5}$, Igor Andreoni ${ }^{1}$ (D), Michael W. Coughlin ${ }^{1,6}$ (D) , Leo P. Singer ${ }^{7,8}$ (D) , Erik C. Kool ${ }^{9}$ (D), Kishalay De ${ }^{1}$, Harsh Kumar ${ }^{10}$, Mouza AlMualla ${ }^{11}$, Yuhan Yao ${ }^{1}$ (D), Mattia Bulla $^{12}$ (ID), Dougal Dobie ${ }^{13,14,15}$ (D), Simeon Reusch ${ }^{3,4}$, Daniel A. Perley ${ }^{16}$ (iD), S. Bradley Cenko ${ }^{7,8}$ (D), Varun Bhalerao ${ }^{10}$ (iD), David L. Kaplan $^{17}$ (D), Jesper Sollerman ${ }^{9}$ (D), Ariel Goobar ${ }^{5}$ (D), Christopher M. Copperwheat ${ }^{16}$ (D), Eric C. Bellm ${ }^{18}$ (iD), G. C. Anupama ${ }^{19}$ (D), Alessandra Corsi ${ }^{20}$ (D) , Samaya Nissanke ${ }^{21}$ (D), Iván Agudo ${ }^{22}$, Ashot Bagdasaryan ${ }^{1}$, Sudhanshu Barway ${ }^{19}$ (iD), Justin Belicki $^{23}$, Joshua S. Bloom ${ }^{24,25}$ (D), Bryce Bolin ${ }^{1}$, David A. H. Buckley ${ }^{26}$, Kevin B. Burdge ${ }^{1}$ (D), Rick Burruss ${ }^{23}$, Maria D. Caballero-García ${ }^{27}$, Chris Cannella ${ }^{1}$, Alberto J. Castro-Tirado ${ }^{22,28}$, David O. Cook ${ }^{29}$ (iD, Jeff Cooke ${ }^{15,30}$ (iD), Virginia Cunningham $^{2}$ (D), Aishwarya Dahiwale ${ }^{1}$, Kunal Deshmukh ${ }^{10}$, Simone Dichiara ${ }^{2,7}$, Dmitry A. Duev ${ }^{1}$ (D), Anirban Dutta ${ }^{19}$, Michael Feeney $^{23}$, Anna Franckowiak ${ }^{3}$ (D), Sara Frederick ${ }^{2}$ (D), Christoffer Fremling ${ }^{1}$ (D), Avishay Gal-Yam ${ }^{31}$ (D), Pradip Gatkine ${ }^{2}$ (iD), Shaon Ghosh $^{32}$ (D), Daniel A. Goldstein ${ }^{1,54}$ (D), V. Zach Golkhou ${ }^{18,33,55}$ (D), Matthew J. Graham ${ }^{1}$ (D) Melissa L. Graham ${ }^{1}$ (D), Matthew J. Hankins ${ }^{1}$ (D), George Helou ${ }^{34}$ (D) , Youdong Hu ${ }^{22,35}$ (D), Wing-Huen Ip ${ }^{36}$, Amruta Jaodand ${ }^{1}$ (D), Viraj Karambelkar ${ }^{1}$, Albert K. H. Kong ${ }^{37}$ (D), Marek Kowalski ${ }^{38,39}$, Maitreya Khandagale ${ }^{10}$, S. R. Kulkarni ${ }^{1}$ (D), Brajesh Kumar ${ }^{19}$ (D), Russ R. Laher ${ }^{34}$ (D), K. L. Li ${ }^{37}$, Ashish Mahabal ${ }^{1,40}$ (D), Frank J. Masci ${ }^{34}$ (D), Adam A. Miller ${ }^{41,42}$ (D), Moses Mogotsi ${ }^{26,43}$, Siddharth Mohite ${ }^{17,56}$, Kunal Mooley $^{1}$ (D), Przemek Mroz ${ }^{1}$, Jeffrey A. Newman ${ }^{44}$ (D), Chow-Choong Ngeow ${ }^{36}$ (D), Samantha R. Oates ${ }^{45}$, Atharva Sunil Patil ${ }^{36}$, Shashi B. Pandey ${ }^{46}$, M. Pavana ${ }^{19}$, Elena Pian ${ }^{47}$ (D), Reed Riddle ${ }^{23}$ (D), Rubén Sánchez-Ramírez ${ }^{48}$,

Yashvi Sharma ${ }^{1}$, Avinash Singh ${ }^{19}$, Roger Smith $^{23}$, Maayane T. Soumagnac ${ }^{49,50}$ (D), Kirsty Taggart ${ }^{16}$, Hanjie Tan ${ }^{36}$,

Anastasios Tzanidakis $^{1}$ (D), Eleonora Troja ${ }^{2,7}$ (D), Azamat F. Valeev ${ }^{51}$, Richard Walters ${ }^{23}$, Gaurav Waratkar ${ }^{10}$, Sara Webb ${ }^{15,30}$ (ID, Po-Chieh $\mathrm{Yu}^{36}$ (D), Bin-Bin Zhang ${ }^{52,53}$ (D), Rongpu Zhou ${ }^{49}$ (D), and Jeffry Zolkower ${ }^{23}$

${ }^{1}$ Division of Physics, Mathematics, and Astronomy, California Institute of Technology, Pasadena, CA 91125, USA

${ }^{2}$ Department of Astronomy, University of Maryland, College Park, MD 20742, USA

${ }^{3}$ Deutsches Elektronen Synchrotron DESY, Platanenallee 6, D-15738 Zeuthen, Germany

${ }^{4}$ Institut für Physik, Humboldt-Universität zu Berlin, D-12489 Berlin, Germany

${ }^{5}$ The Oskar Klein Centre, Department of Physics, Stockholm University, AlbaNova, SE-106 91 Stockholm, Sweden

${ }^{6}$ School of Physics and Astronomy, University of Minnesota, Minneapolis, MN 55455, USA

${ }^{7}$ Astrophysics Science Division, NASA Goddard Space Flight Center, MC 661, Greenbelt, MD 20771, USA

8 Joint Space-Science Institute, University of Maryland, College Park, MD 20742, USA

${ }^{9}$ The Oskar Klein Centre, Department of Astronomy, Stockholm University, AlbaNova, SE-106 91 Stockholm, Sweden ${ }^{10}$ Indian Institute of Technology Bombay, Powai, Mumbai 400076, India

${ }^{11}$ American University of Sharjah, Physics Department, P.O. Box 26666, Sharjah, UAE

${ }^{12}$ Nordita, KTH Royal Institute of Technology and Stockholm University, Roslagstullsbacken 23, SE-106 91 Stockholm, Sweden

${ }^{13}$ Sydney Institute for Astronomy, School of Physics, University of Sydney, NSW 2006, Australia

${ }^{14}$ CSIRO Astronomy and Space Science, P.O. Box 76, Epping, NSW 1710, Australia

15 Australian Research Council Centre of Excellence for Gravitational Wave Discovery (OzGrav), Swinburne University of Technology, Hawthorn, VIC 3122 , Australia

${ }^{16}$ Astrophysics Research Institute, Liverpool John Moores University, IC2, Liverpool Science Park, 146 Brownlow Hill, Liverpool L3 5RF, UK

${ }^{17}$ Center for Gravitation, Cosmology and Astrophysics, Department of Physics, University of Wisconsin-Milwaukee, P.O. Box 413, Milwaukee, WI 53201, USA

${ }^{18}$ DIRAC Institute, Department of Astronomy, University of Washington, 3910 15th Avenue NE, Seattle, WA 98195, USA

${ }^{9}$ Indian Institute of Astrophysics, II Block Koramangala, Bengaluru 560034, India

${ }^{20}$ Department of Physics and Astronomy, Texas Tech University, Box 1051, Lubbock, TX 79409-1051, USA

${ }^{21}$ Center of Excellence in Gravitation and Astroparticle Physics, University of Amsterdam, Netherlands

${ }^{22}$ Instituto de Astrofísica de Andalucía (IAA-CSIC), Glorieta de la Astronomía s/n, E-18008, Granada, Spain

${ }^{23}$ Caltech Optical Observatories, California Institute of Technology, Pasadena, CA 91125, USA

${ }^{24}$ Department of Astronomy, University of California, Berkeley, CA 94720, USA

${ }^{25}$ Physics Division, Lawrence Berkeley National Laboratory, 1 Cyclotron Road, MS 50B-4206, Berkeley, CA 94720, USA

${ }^{26}$ South African Astronomical Observatory, P.O. Box 9, Observatory 7935, Cape Town, South Africa

${ }^{27}$ Astronomical Institute of the Academy of Sciences, Boční II 1401, CZ-14100 Praha 4, Czech Republic

${ }^{28}$ Unidad Asociada Departamento de Ingeniería de Sistemas y Automática, E.T.S. de Ingenieros Industriales, Universidad de Málaga, Spain

${ }^{29}$ Caltech/IPAC, California Institute of Technology, 1200 East California Boulevard, Pasadena, CA 91125, USA

${ }^{30}$ Centre for Astrophysics and Supercomputing, Swinburne University of Technology, Hawthorn, VIC 3122, Australia

${ }^{31}$ Edna and K.B. Weissman Building of Physical Sciences, Weizmann Institute of Science, Rehovot 76100, Israel

${ }^{32}$ Department of Physics and Astronomy, Montclair State University, Montclair, NJ 07043, USA

${ }^{33}$ The eScience Institute, University of Washington, Seattle, WA 98195, USA

${ }_{35}^{34}$ IPAC, California Institute of Technology, 1200 East California Boulevard, Pasadena, CA 91125, USA

${ }^{35}$ Universidad de Granada, Facultad de Ciencias Campus Fuentenueva S/N CP E-18071 Granada, Spain

${ }^{36}$ Graduate Institute of Astronomy, National Central University, 32001, Taiwan

${ }^{37}$ Institute of Astronomy, National Tsing Hua University, Hsinchu 30013, Taiwan

${ }^{38}$ Institute of Physics, Humboldt-Universität zu Berlin, Newtonstr. 15, D-124 89 Berlin, Germany

${ }^{39}$ Deutsches Elektronensynchrotron, Platanenallee 6, D-15738, Zeuthen, Germany

${ }^{40}$ Center for Data Driven Discovery, California Institute of Technology, Pasadena, CA 91125, USA

${ }^{41}$ Center for Interdisciplinary Exploration and Research in Astrophysics (CIERA) and Department of Physics and Astronomy, Northwestern University, 1800 Sherman Road, Evanston, IL 60201, USA

${ }^{42}$ The Adler Planetarium, Chicago, IL 60605, USA

${ }^{43}$ Southern African Large Telescope Foundation, P.O. Box 9, Observatory 7935, Cape Town, South Africa

${ }_{44}$ Department of Physics and Astronomy and PITT PACC, University of Pittsburgh, PA 15260, USA 


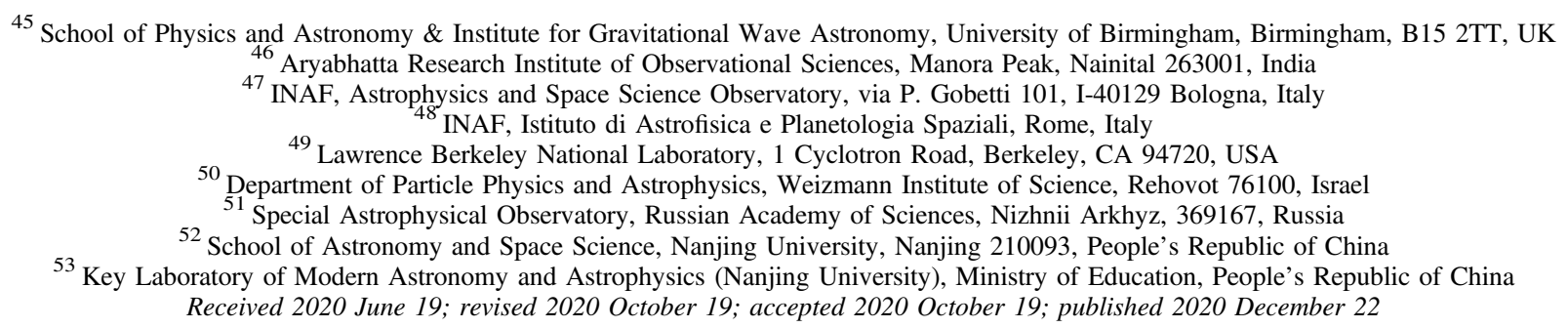

\begin{abstract}
We present a systematic search for optical counterparts to 13 gravitational wave (GW) triggers involving at least one neutron star during LIGO/Virgo's third observing run (O3). We searched binary neutron star (BNS) and neutron star black hole (NSBH) merger localizations with the Zwicky Transient Facility (ZTF) and undertook follow-up with the Global Relay of Observatories Watching Transients Happen (GROWTH) collaboration. The GW triggers had a median localization area of $4480 \mathrm{deg}^{2}$, a median distance of $267 \mathrm{Mpc}$, and false-alarm rates ranging from 1.5 to $10^{-25} \mathrm{yr}^{-1}$. The ZTF coverage in the $g$ and $r$ bands had a median enclosed probability of 39\%, median depth of $20.8 \mathrm{mag}$, and median time lag between merger and the start of observations of $1.5 \mathrm{hr}$. The O3 follow-up by the GROWTH team comprised 340 UltraViolet/Optical/InfraRed (UVOIR) photometric points, 64 OIR spectra, and three radio images using 17 different telescopes. We find no promising kilonovae (radioactivitypowered counterparts), and we show how to convert the upper limits to constrain the underlying kilonova luminosity function. Initially, we assume that all GW triggers are bona fide astrophysical events regardless of falsealarm rate and that kilonovae accompanying BNS and NSBH mergers are drawn from a common population; later, we relax these assumptions. Assuming that all kilonovae are at least as luminous as the discovery magnitude of GW170817 (-16.1 mag), we calculate that our joint probability of detecting zero kilonovae is only $4.2 \%$. If we assume that all kilonovae are brighter than -16.6 mag (the extrapolated peak magnitude of GW170817) and fade at a rate of $1 \mathrm{mag} \mathrm{day}^{-1}$ (similar to GW170817), the joint probability of zero detections is $7 \%$. If we separate the NSBH and BNS populations based on the online classifications, the joint probability of zero detections, assuming all kilonovae are brighter than $-16.6 \mathrm{mag}$, is $9.7 \%$ for NSBH and $7.9 \%$ for BNS mergers. Moreover, no more than $<57 \%(<89 \%)$ of putative kilonovae could be brighter than -16.6 mag assuming flat evolution (fading by $1 \mathrm{mag}$ $\mathrm{day}^{-1}$ ) at the $90 \%$ confidence level. If we further take into account the online terrestrial probability for each $\mathrm{GW}$ trigger, we find that no more than $<68 \%$ of putative kilonovae could be brighter than -16.6 mag. Comparing to model grids, we find that some kilonovae must have $M_{\mathrm{ej}}<0.03 M_{\odot}, X_{\mathrm{lan}}>10^{-4}$, or $\phi>30^{\circ}$ to be consistent with our limits. We look forward to searches in the fourth GW observing run; even 17 neutron star mergers with only $50 \%$ coverage to a depth of -16 mag would constrain the maximum fraction of bright kilonovae to $<25 \%$.
\end{abstract}

Unified Astronomy Thesaurus concepts: Neutron stars (1108); Black holes (162); Gravitational waves (678); Nucleosynthesis (1131); R-process (1324); Compact objects (288); Spectroscopy (1558); Sky surveys (1464); Photometry (1234)

\section{Introduction}

Gravitational-wave (GW) astrophysics is achieving a new frontier every 2 yr. On 2015 September 14, the Advanced LIGO/Virgo Collaboration (LVC) celebrated the revolutionary discovery of GWs from merging massive stellar black holes (BBHs; Abbott et al. 2016). On 2017 August 17, the physics and astronomy communities jointly celebrated the detection of GWs from the first binary neutron star (BNS) merger that lit up the entire electromagnetic (EM) spectrum (Abbott et al. 2017a, 2017b; Coulter et al. 2017; Evans et al. 2017; Goldstein et al. 2017; Haggard et al. 2017; Hallinan et al. 2017; Margutti et al. 2017; Troja et al. 2017; Kasliwal et al. 2019b). On 2019 April 26, the first candidate neutron star black hole (NSBH) merger was announced by Advanced LIGO/Virgo (Ligo Scientific Collaboration \& VIRGO Collaboration 2019a, 2019b), and since then, there have been eight additional candidate NSBH events.

Unlike a BNS system, the very existence of an NSBH binary was observationally unconstrained. No pulsar in the Milky

\footnotetext{
${ }_{55}^{54}$ Hubble Fellow.

55 Moore-Sloan, WRF, and DIRAC Fellow.

${ }^{56}$ LSSTC Data Science Fellow.
}

Way is known to have a black hole companion. A compact BNS merger has a viable stellar evolutionary formation channel (Tauris et al. 2015), since a few ultrastripped supernovae (SNe) have been seen (De et al. 2018; Nakaoka et al. 2020; Yao et al. 2020). On the other hand, it has been argued that the supermassive black holes in the nuclei of galaxies assist in the formation of compact NSBH (and BBH) systems by the eccentric Kozai-Lidov (EKL) mechanism (Naoz 2016; Stephan et al. 2019). Unlike a BNS merger, for which GW170817 serves as the Rosetta Stone of what to look for, theoretical predictions of the EM counterparts to NSBH mergers span a wide spectrum, depending on the system parameters (e.g., mass ratio, spin of the black hole, equation of state of the neutron star). While some scenarios predict that the neutron star is swallowed whole by the black hole and there is no EM emission, others predict a luminous kilonova where, compared to the BNS case, more lanthanide-rich material is ejected dynamically while comparable masses are ejected from the disk (e.g., Rosswog 2005; Foucart 2012; Hotokezaka et al. 2013; Kiuchi et al. 2015; Kawaguchi et al. 2016; Kasen et al. 2017; Kruckow et al. 2018; Broekgaarden et al. 2019; Nakar 2020; Fernández et al. 2020). 
LIGO/Virgo's third observing run (O3; from 2019 April to 2020 March) has yielded real-time alerts on six BNS mergers and nine NSBH mergers. Alerts and localization maps were publicly released within minutes to a few hours after the mergers. Updates to localization maps and false-alarm rates (FARs) were released days to weeks after the mergers. The median localization was $4480 \mathrm{deg}^{2}$. The median distance to BNS mergers was $214 \mathrm{Mpc}$, and that to NSBH mergers was $377 \mathrm{Mpc}$.

Given that the optical counterpart of GW170817 was first observed only $10.8 \mathrm{hr}$ after merger, there is considerable debate on how the early emission evolves. Different models predict different early evolution (e.g., Drout et al. 2017; Kasliwal et al. 2017; Arcavi 2018; Piro \& Kollmeier 2018; Waxman et al. 2018). Thanks to the low latency in the public O3 alerts, prompt follow-up was undertaken. Despite the localizations being coarser and the distances being further than expected (Abbott et al. 2018), the Global Relay of Observatories Watching Transients Happen $\left(\mathrm{GROWTH}^{57}\right)$ collaboration undertook systematic searches and extensive follow-up of every trigger with a worldwide network of telescopes. We used three discovery engines, the Zwicky Transient Facility (ZTF; Bellm et al. 2018; Masci et al. 2018; Graham et al. 2019), Palomar Gattini-IR (PGIR; Moore \& Kasliwal 2019; De et al. 2020a), and Dark Energy Camera (DECam; Goldstein et al. 2019), and a suite of 17 follow-up facilities. Candidate counterparts and follow-up results from these searches were promptly announced via Gamma-ray Coordinates Network (GCN) circulars. In addition to GROWTH, several teams undertook wide-field searches for optical counterparts in O3, including Electromagnetic counterparts of Gravitational wave sources at the Very Large Telescope (ENGRAVE; Levan 2020), Global Rapid Advanced Network Devoted to the Multi-messenger Addicts (GRANDMA; Antier et al. 2020), Gravitational-wave Optical Transient Observer (GOTO; Gompertz et al. 2020), All Sky Automated Survey for SuperNovae (ASAS-SN; Shappee et al. 2014), Asteroid Terrestrial Last Alert System (ATLAS; Tonry et al. 2018), Panoramic Survey Telescope and Rapid Response System (PanSTARRS; Chambers et al. 2016), MASTER-Net (Lipunov et al. 2017), Searches after Gravitational Waves Using ARizona Observatories (SAGUARO; Lundquist et al. 2019), Dark Energy Survey Gravitational Wave Collaboration (DES-GW; Soares-Santos et al. 2017), Burst Optical Observer and Transient Exploring System (BOOTES), KM3Net, ${ }^{58}$ and VINROUGE ${ }^{59}$ (PI: Tanvir). We also undertook a wide-field radio search with the Australian Square Kilometre Array Pathfinder (ASKAP; Dobie et al. 2019).

This paper focuses on events that contain at least one neutron star; see Graham et al. (2020) for our candidate counterpart to a binary black hole merger. The LVC published GW190425 as a confirmed astrophysical BNS with a total system mass of $3.4 M_{\odot}$ (Abbott et al. 2020a). The LVC also published GW190814 as a confirmed astrophysical merger of a $23 M_{\odot}$ black hole with a $2.6 M_{\odot}$ compact object (Abbott et al. 2020b). While we await the final $\mathrm{LVC}$ results on the candidature and binary parameters of all other merger candidates from $\mathrm{O} 3$, we use the classifications and parameters released via GCN circulars. We refer to GW190814 and GW190425 as "sources" or "confirmed events" and use a

\footnotetext{
57 http://growth.caltech.edu/

58 https://www.km3net.org/

59 https://www.star.le.ac.uk/nrt3/VINROUGE/
}

"GW" prefix in the name. We refer to all others as "triggers" or "candidate events" with an "S" prefix in the name. We refer to the full set as "events."

We have previously published our search results for the highestsignificance mergers: GW190425 (Coughlin et al. 2019d), GW190814 (Dobie et al. 2019; Andreoni et al. 2020b), and S2001015ae and S200115j (Anand et al. 2020). Here we focus on ZTF searches of the full set of $\mathrm{O} 3$ triggers and the implications of the joint nondetection of kilonovae from all merger candidates. In Section 2, we summarize the GW trigger selection criteria. In Section 3, we detail the discovery, follow-up, and rejection of candidate optical counterparts. In Section 4, we examine the model-independent implications of the luminosity function of kilonovae. In Section 5, we summarize our key results and look ahead to future GW observing runs.

\section{Summary of GW Triggers}

During the third LIGO/Virgo observing run, we triggered target-of-opportunity (ToO) searches based on the following criteria: (a) an initial classification with the highest probability of either BNS, NSBH, or MassGap; (b) if MassGap, then a nonzero probability of containing a neutron star; and (c) a visibility and mapping speed allowing us to observe $>30 \%$ of the initial BAYESTAR sky map (Singer \& Price 2016) within $24 \mathrm{hr}$ of merger.

A total of $15 \mathrm{GW}$ events satisfied criteria (a) and (b). In Table 1, we summarize $13 \mathrm{GW}$ triggers during $\mathrm{O} 3$ for which we obtained either serendipitous or triggered coverage with the ZTF (we did not get any ZTF data on S190510g, as the sky position was too far south, or S190924h, as the sky position was too close to the Moon). In Figures 1-3, we show the ZTF coverage overlaid on the GW localization contours. Since the public ZTF survey systematically covers the accessible northern sky at an average cadence of 3 days to a median depth of $20.5 \mathrm{mag}$ (Bellm et al. 2018), we "serendipitously" covered several GW sky maps. Serendipitous coverage contributed to more than $30 \%$ enclosed probability for the following triggers: GW190814, S190910d, S190910h, S190923y, S190930t, S200105ae, and S200213t. To improve depth/coverage/ response time, we triggered ZTF ToO observations for 11 out of 15 events (and undertook DECam searches for three events; see Andreoni et al. 2019c, 2020b; Goldstein et al. 2019). Our triggered $\mathrm{ToO}$ observations optimized the trade-off between depth (more exposure time per pointing) and coverage of the localization map (more pointings to enclose more probability) using the gwemopt algorithm. A detailed case study of the ToO observations can be found in Coughlin et al. (2019d) and Anand et al. (2020). For S191205ah, our triggered observations were not completed due to bad weather, and only a small fraction was covered serendipitously. For S190910h, given the coarse localization, we relied only on serendipitous coverage as part of regular ZTF operations. For S190923y, given the large time lag between GW alert and first target visibility, we also relied only on serendipitous coverage.

The location of Palomar Observatory relative to LIGO's quadrupolar antenna sensitivity pattern helps minimize the time lag to respond to triggers in real time (see Figure 4); the latency to first observation was between $11 \mathrm{~s}$ and $13.7 \mathrm{hr}$. (The lowest latency of $11 \mathrm{~s}$ was enabled by serendipitous coverage.) As predicted by simulations (Nissanke et al. 2013; Kasliwal \& Nissanke 2014), all (but one) GW public alerts were accessible from Palomar Observatory, and more than half could be 
Table 1

Summary of ZTF Follow-up of $13 \mathrm{GW}$ Triggers in O3

\begin{tabular}{|c|c|c|c|c|c|c|c|c|c|}
\hline Name & $\operatorname{FAR}\left(P_{t}\right)$ & Localization & Distance & Class & $P_{1}$ & $P_{2}$ & Time Lag & Depth & $\overline{E(B-V)}$ \\
\hline GW190425 & 1 per 69,000 yr $(1 \%)$ & $7461 \mathrm{deg}^{2}$ & $156 \pm 41 \mathrm{Mpc}$ & BNS & $24.13 \%(45.92 \%)$ & $23.90 \%(44.62 \%)$ & $0.003 \mathrm{hr}$ & 21.5 & 0.03 \\
\hline S190426c & 1 per $1.6 \mathrm{yr}(58 \%)$ & $1131 \mathrm{deg}^{2}$ & $377 \pm 100 \mathrm{Mpc}$ & $\mathrm{NSBH}$ & $52.33 \%(59.69 \%)$ & $51.57 \%(57.40 \%)$ & $13.06 \mathrm{hr}$ & 21.5 & 0.34 \\
\hline GW190814 & 1 per $10^{25}$ yr $(1 \%)$ & $23 \operatorname{deg}^{2}$ & $267 \pm 52 \mathrm{Mpc}$ & $\mathrm{NSBH}$ & $88.57 \%(87.00 \%)$ & $78.37 \%(70.60 \%)$ & $0.00 \mathrm{hr}$ & 21.0 & 0.02 \\
\hline S190901ap & 1 per 4.5 yr $(14 \%)$ & $14,753 \mathrm{deg}^{2}$ & $241 \pm 79 \mathrm{Mpc}$ & BNS & $56.94 \%(50.67 \%)$ & $49.39 \%(42.76 \%)$ & $3.61 \mathrm{hr}$ & 21.0 & 0.03 \\
\hline S190910d & 1 per 8.5 yr $(2 \%)$ & $2482 \mathrm{deg}^{2}$ & $632 \pm 186 \mathrm{Mpc}$ & $\mathrm{NSBH}$ & $32.99 \%(42.50 \%)$ & $31.17 \%(39.64 \%)$ & $1.51 \mathrm{hr}$ & 20.3 & 0.04 \\
\hline S190910h & 1 per 0.9 yr $(39 \%)$ & $24,264 \mathrm{deg}^{2}$ & $230 \pm 88 \mathrm{Mpc}$ & BNS & $33.26 \%(42.95 \%)$ & $28.92 \%(38.44 \%)$ & $0.015 \mathrm{hr}$ & 20.4 & 0.08 \\
\hline S190923y & 1 per 0.67 yr $(32 \%)$ & $2107 \mathrm{deg}^{2}$ & $438 \pm 133 \mathrm{Mpc}$ & $\mathrm{NSBH}$ & NA $(38.99 \%)$ & NA $(19.22 \%)$ & $13.73 \mathrm{hr}$ & 20.1 & 0.09 \\
\hline $\mathrm{S} 190930 \mathrm{t}$ & 1 per $2.0 \mathrm{yr}(26 \%)$ & $24,220 \mathrm{deg}^{2}$ & $108 \pm 38 \mathrm{Mpc}$ & $\mathrm{NSBH}$ & NA $(50.63 \%)$ & NA $(43.42 \%)$ & $11.91 \mathrm{hr}$ & 21.1 & 0.05 \\
\hline S191205ah & 1 per 2.5 yr $(7 \%)$ & $6378 \operatorname{deg}^{2}$ & $385 \pm 164 \mathrm{Mpc}$ & $\mathrm{NSBH}$ & NA $(5.68 \%)$ & NA $(4.85 \%)$ & $10.66 \mathrm{hr}$ & 17.9 & 0.04 \\
\hline S191213g & 1 per 0.89 yr $(23 \%)$ & $4480 \mathrm{deg}^{2}$ & $201 \pm 81 \mathrm{Mpc}$ & BNS & $27.50 \%(0.80 \%)$ & $25.10 \%(0.09 \%)$ & $0.013 \mathrm{hr}$ & 20.4 & 0.30 \\
\hline S200105ae & NA $(97 \%)$ & $7373 \mathrm{deg}^{2}$ & $283 \pm 74 \mathrm{Mpc}$ & $\mathrm{NSBH}$ & $52.39 \%(56.40 \%)$ & $43.99 \%(47.96 \%)$ & $9.96 \mathrm{hr}$ & 20.2 & 0.05 \\
\hline $\mathrm{S} 200115 \mathrm{j}$ & 1 per 1513 yr $(1 \%)$ & $765 \mathrm{deg}^{2}$ & $340 \pm 79 \mathrm{Mpc}$ & $\mathrm{NSBH}$ & $22.21 \%(34.92 \%)$ & $15.76 \%(18.17 \%)$ & $0.24 \mathrm{hr}$ & 20.8 & 0.13 \\
\hline $\mathrm{S} 200213 \mathrm{t}$ & 1 per 1.8 yr $(37 \%)$ & $2326 \mathrm{deg}^{2}$ & $201 \pm 80 \mathrm{Mpc}$ & BNS & $72.17 \%(79.29 \%)$ & $70.48 \%(76.08 \%)$ & $0.40 \mathrm{hr}$ & 21.2 & 0.19 \\
\hline
\end{tabular}

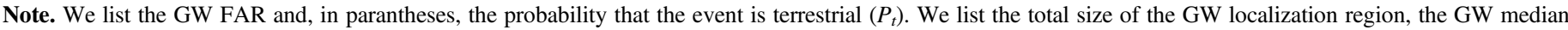





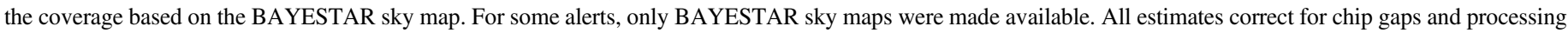

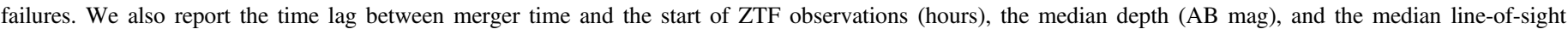
extinction.

followed up within $4 \mathrm{hr}$ of the merger. Throughout the paper, we only use enclosed probability based on the LALInference sky map, as it is deemed more accurate (Veitch et al. 2015), when available. The LALInference sky maps were mostly released only after our observations were completed. Hence, the enclosed probability estimates were systematically lower than those estimated by the observation plan based on the initial BAYESTAR sky maps (see Table 1).

The process for triggering ToO observations for a survey system like the ZTF differs from traditional telescopes, as it involves halting the ongoing survey observations and scheduling observations of only certain fields as selected by an observation plan. Observation plans are generated by gwemopt, ${ }^{60}$ a code base for optimizing galaxy-targeted and synoptic searches within GW sky maps (Coughlin et al. 2018, 2019a). Over the course of $\mathrm{O} 3$, we implemented several improvements to the existing code framework, including additional features that allow us to strategically handle sky maps spanning thousands of square degrees, slice sky maps by R.A. and schedule slices separately, and balance coverage in multiple filters. These improvements, among others, are described in Almualla et al. (2020). All of our triggered follow-up of GW events, gammaray bursts (GRBs; T. Ahumada et al. 2020, in preparation), and high-energy neutrino events (Stein et al. 2020) occurs through a user interface called the GROWTH ToO Marshal, ${ }^{61}$ a database designed to ingest GCN circulars, display event properties and sky maps, design plans, trigger observations, query for candidates within the observed region, and retrieve summary statistics for completed observations, including probability covered and median depth (Coughlin et al. 2019a; Kasliwal et al. 2019a).

\section{Investigating Candidate Counterparts}

Our candidate vetting methodology has continued to improve over the past few years, starting with Fermi afterglow searches

\footnotetext{
60 https://github.com/mcoughlin/gwemopt

61 https://github.com/growth-astro/growth-too-marshal
}

(Singer et al. 2015), to BBH searches in O1 (Kasliwal et al. 2016), to BNS and NSBH searches in O3 (Coughlin et al. 2019a; Anand et al. 2020). We graphically summarize the candidate vetting process in Figure 5. Here we first discuss the prompt vetting procedure that quickly led to a GCN circular announcing candidate counterparts (Section 3.1). Next, we discuss follow-up of the candidates to discern their nature (Section 3.2). Finally, we discuss a deeper offline search to look for any missed candidates (Section 3.3).

\subsection{Initial Transient Vetting}

For each of the $13 \mathrm{GW}$ triggers followed up by ZTF, we systematically identified transient candidates within the localization region and ruled them out using various metrics. Below, we summarize the transient filtering process and results from our candidate vetting.

The GROWTH team has three independent database systems to retrieve interesting objects in real time: the GROWTH Marshal (Kasliwal et al. 2019a), the Kowalski ${ }^{62}$ system (Duev et al. 2019), and the Alert Management, Photometry and Evaluation of Lightcurves (AMPEL) system (Soumagnac \& Ofek 2018; Nordin et al. 2019). Each platform retrieves a stream of AVRO packet alerts (Patterson et al. 2019) containing significant object detections identified by the ZTF image subtraction pipeline, defined as a $>5 \sigma$ change in brightness relative to a reference image (Masci et al. 2018). Each of these objects undergoes a series of filtering steps in order to identify candidates that could be interesting to pursue for follow-up. The following criteria were common for all three queries.

1. Positive subtraction. The object must have brightened relative to the reference image.

2. Astrophysical. The object must have a real bogus (rb) score $>0.25$ or a deep learning (drb) score $>0.8$ (Duev et al. 2019; Mahabal et al. 2019) for it to be considered astrophysical.

\footnotetext{
62 https://github.com/dmitryduev/kowalski
} 


\section{GW190814}

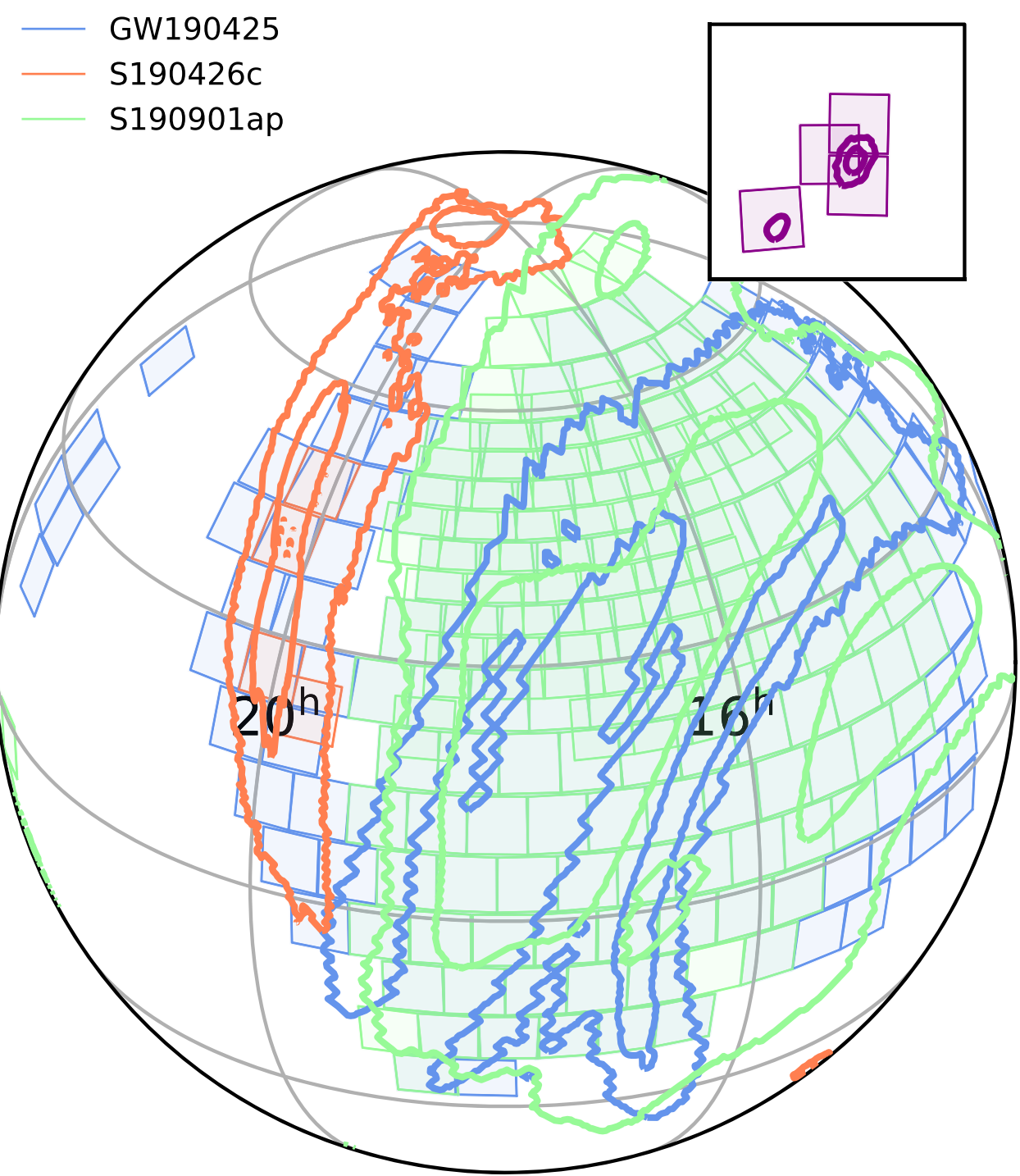

Figure 1. The ZTF coverage maps of two BNS triggers (S190901ap and GW190425) and two NSBH triggers (S190426c and GW190814) during O3 of LIGO/Virgo. Each square represents a ZTF pointing, and the solid line denotes the latest available GW $90 \%$ localization contour. Despite both BNS triggers being localized to a $\pi$ of the sky, the ZTF was able to map the accessible localization area in a few hours.

3. Not stellar. The object must be $>2^{\prime \prime}$ away from a cataloged point source in the PanSTARRS Point Source Catalog (Tachibana \& Miller 2018).

4. Far from a bright source. The object must be at least $20^{\prime \prime}$ away from a bright $\left(m_{\mathrm{AB}}<15 \mathrm{mag}\right)$ star to avoid blooming artifacts.

5. Not moving. The object must have at least two detections separated by at least 15 minutes to reject asteroids (moves $<4^{\prime \prime} \mathrm{hr}^{-1}$ )

6. No previous history. The object must not have any historical detections in the ZTF alert stream prior to the $\mathrm{GW}$ merger time.

While the GROWTH Marshal queried all fields triggered as part of the ToO search, the Kowalski and AMPEL queries searched for candidates in both serendipitous and triggered data within the $95 \%$ contour of the latest sky map that was available. The AMPEL query ${ }^{63}$ had further image quality cuts performed

${ }^{63}$ https://github.com/robertdstein/ampel_followup_pipeline to reject poor subtractions based on morphology, an additional cut based on proximity to known solar system objects, and another cut based on cross-matching to the Gaia Data Release 2 (DR2) catalog and PS1 to identify likely stellar sources.

All candidates that passed the filtering criteria were saved to the GROWTH Marshal for further vetting in real time by a dedicated team of scanners. If a transient was consistent with the nucleus of a galaxy and the mid-infrared colors (based on the Wide-field Infrared Survey Explorer (WISE) catalog; Wright et al. 2010) of the host galaxy were consistent with active galactic nuclei (AGNs), the candidate was deemed unrelated.

All viable candidates were promptly announced to the worldwide community via GCN circulars, and many teams (not only GROWTH) triggered follow-up observations for many of our candidates. ${ }^{64}$ Using the GROWTH Marshal system, we prioritized and triggered follow-up of candidates that exhibited

\footnotetext{
${ }^{64}$ The GROWTH collaboration posted 82 GCNs during O3. An additional 151 GCNs refer to follow-up of ZTF objects by other teams.
} 

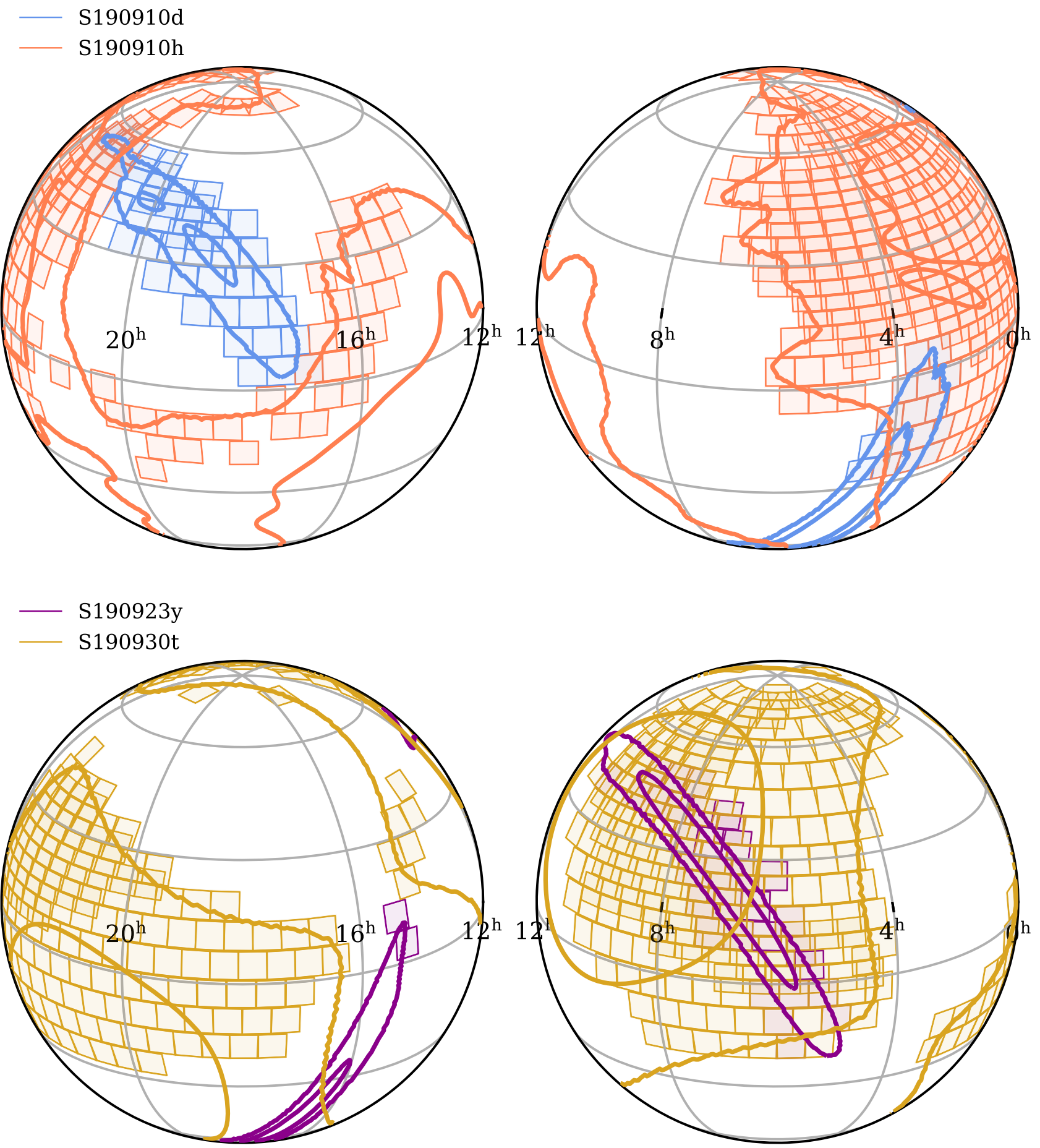

Figure 2. Top: ZTF coverage maps of the two same-day triggers occurring on September 10 (S190910d and S190910h) during O3 of LIGO/Virgo. Given the spatial and temporal overlap of these two GW triggers, some field observations contributed to coverage of both. Bottom: ZTF coverage maps of two NSBH triggers (S190923y and S190930t) during O3. Each square represents a ZTF pointing, and the solid line denotes the latest available GW 90\% localization contour.

rapid photometric evolution (faster than $0.3 \mathrm{mag}$ day $^{-1}$ ), showed red colors, or were close to a host galaxy with a redshift consistent with the GW distance constraint.

\subsection{Examining Promising Candidate Counterparts with Additional Follow-up}

We now briefly describe how we ruled out the association between vetted counterpart candidates and the GW event. A detailed account of every candidate announced via GCN is in Appendix B.
The GROWTH team obtained follow-up with the following facilities to characterize the photometric and/or spectroscopic evolution: the Liverpool Telescope (LT; Steele et al. 2004), Lowell Discovery Telescope (LDT, ${ }^{65}$ formerly known as the Discovery Channel Telescope), Las Cumbres Observatory (LCO; Brown et al. 2013), Apache Point Observatory (APO; Huehnerhoff et al. 2016), Kitt Peak EMCCD Demonstrator (KPED; Coughlin et al. 2019b), Lulin One-meter Telescope

\footnotetext{
${ }^{65}$ https://lowell.edu/research/research-facilities/4-3-meter-ldt/
} 

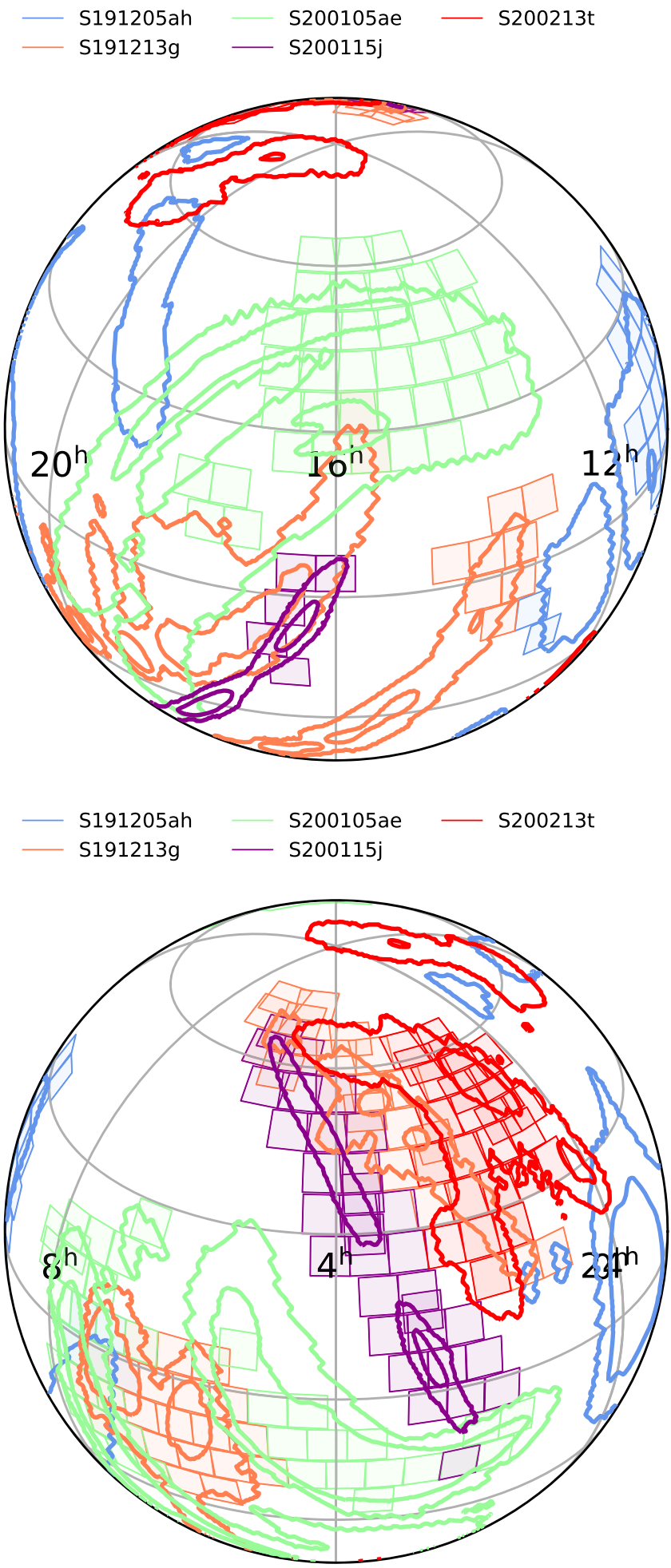

Figure 3. The ZTF coverage maps of the triggers during the second half of $\mathrm{O} 3$ of LIGO/Virgo: S191205ah, S191213g, S200105ae, S200115j, and S200213t. The top and bottom panels show opposite sides of the globe. Each square represents a ZTF pointing, and the solid line denotes the latest available GW $90 \%$ localization contour.

(LOT; Huang et al. 2005), GROWTH-India telescope (GIT; ${ }^{66}$ V. Bhalerao et al. 2020, in preparation), Palomar 60 inch

\footnotetext{
${ }^{66}$ https://sites.google.com/view/growthindia/
}



Figure 4. Distribution of response time, defined as the time lag between merger time and earliest possible observation time at a given site, for all 15 BNS/ $\mathrm{NSBH}$ triggers in O3. We define that observations can begin when at least $30 \%$ of the enclosed probability of a GW localization contour is above airmass 2.0 and the Sun is $12^{\circ}$ below the horizon at a given site. The size of the filled circles scales with the number of triggers in each time bin. Note that the location of Palomar Observatory enables a response to more triggers than CTIO overall (13 vs. 10 triggers) and a larger number (seven vs. two triggers) within $4 \mathrm{hr}$ after merger.

telescope (P60; Cenko et al. 2006), Palomar 200 inch Hale Telescope $^{67}$ (P200), Keck Observatory, ${ }^{68}$ Gemini Observatory, ${ }^{69}$ Southern African Large Telescope ${ }^{70}$ (SALT), Himalayan Chandra Telescope ${ }^{71}$ (HCT), and Gran Telescopio Canarias $^{72}$ (GTC). Figures 6 and 7 illustrate examples of follow-up by the GROWTH team on some ZTF candidates. The specific instrument configurations and data reduction methods are described in Appendix A.

The follow-up observations include both photometric and spectroscopic data. Moreover, the association of a candidate with a GW trigger was rejected if its properties fell into one or more of the categories described as follows.

1. Inconsistent spectroscopic classification. We ruled out candidates that could be spectroscopically classified as $\mathrm{SNe}$, AGNs, cataclysmic variables (CVs), and other flare stars. We used SNID (Blondin \& Tonry 2007) and dash (Muthukrishna et al. 2019) to classify the SNe and AGNs found in our searches. The CVs and variable stars often showed hydrogen features at zero redshift.

2. Inconsistent distance. We ruled out candidates whose spectroscopic redshift was not consistent with the GW distance within $2 \sigma$. We cross-matched the transient positions with the Census of the Local Universe (CLU; Cook et al. 2019) galaxy catalog and the NASA Extragalactic Database (NED) to look up host redshifts where available. We also cross-matched the candidates against the Photometric Redshifts Legacy Survey (PRLS; Zhou et al. 2020) catalog and reported the photometric redshifts when the spectroscopic redshift was unavailable.

\footnotetext{
$\overline{67}$ https://www.astro.caltech.edu/palomar/about/telescopes/hale.html

${ }^{68}$ http://www.keckobservatory.org/

69 http://www.gemini.edu/

${ }^{70}$ https://www.salt.ac.za/

$71 \mathrm{https}: / /$ www.iiap.res.in/?q=telescope_iao

72 http://www.gtc.iac.es/gtc/gtc.php
} 




Figure 5. Flowchart to show how our candidate vetting funneled from a large number of spatially and temporally consistent alerts, to a smaller number of candidates that deem human vetting, to an even smaller number of candidates that warrant detailed follow-up characterization over the course of O3.

3. Slow photometric evolution. As kilonovae are expected to evolve faster than $\mathrm{SNe}$, we ruled out candidates that evolved slower than 0.3 mag day $^{-1}$. We used ForcePhot $^{73}$ (Yao et al. 2019), a forced photometry package, to examine the transient light curves. To quantify the evolution of a given transient, we define the parameter $\alpha_{f}=\Delta m / \Delta t\left[\right.$ mag day $\left.^{-1}\right]$, where $f$ corresponds to the filter used to determine the variation in magnitude $(\Delta m)$ over time $(\Delta t)$. A positive $\alpha$ indicates a fading source, while a negative $\alpha$ describes a rising source. The baseline $(\Delta t)$ is defined to be the number of days it takes an object to rise from its discovery to its peak magnitude $(\alpha<0)$ or the number of days it takes the transient to fade from peak to undetectable by ZTF $(\alpha>0)$. We used a minimum time baseline of 3 days to compute slopes.

4. Outside of the latest LALInference map. The majority of the candidates were selected and announced via GCN based on the promptly available BAYESTAR map (Singer \& Price 2016). When the LALInference map was made available, if a candidate was outside the $90 \%$ probability contour, we rejected it.

5. Artifacts. Most of the ZTF ghosts and artifacts are well known (Bellm et al. 2018; Masci et al. 2018) ${ }^{74}$ and masked automatically. Additionally, we take further precautions by ignoring transients close to bright stars in our initial vetting. However, for example, our extensive analysis revealed a subtle gain mismatch in the reference images that posed as a faint and fast

\footnotetext{
73 https://github.com/yaoyuhan/ForcePhotZTF

${ }^{74}$ http://nesssi.cacr.caltech.edu/ZTF/Web/Ghosts.html
}

transient (see discussion related to ZTF19aassfws in Appendix B). All references for ToOs were rebuilt after this artifact was identified.

6. Asteroids. Sometimes slow-moving asteroids, especially near stationary points, can mimic a fast-fading transient (Jedicke et al. 2016). For these objects, either a more careful inspection of the centroids or movement in follow-up imaging served as the reason for rejection.

7. Previous activity. Candidates were rejected if they showed previous detections prior to the GW merger time in other surveys, e.g., the Catalina Real Time Survey (CRTS; Djorgovski et al. 2011), Palomar Transient Factory (PTF; Law et al. 2009), intermediate Palomar Transient Factory (iPTF; Cao et al. 2016; Masci et al. 2017), and PS1 (Tachibana \& Miller 2018).

Some candidates prompted panchromatic follow-up. We followed up five candidates in the ultraviolet and X-ray with the Neil Gehrels Swift Observatory (see the Appendix for details). We followed up two candidates in the radio with the Arcminute Microkelvin Imager (AMI) and one with the Karl G. Jansky Very Large Array (VLA; see the Appendix for details). All candidates, grouped by GW trigger, are listed in Tables $2-10$, along with their respective rejection criteria.

\subsection{Candidates from Deeper Offline Searches}

We complemented our real-time analysis described above with a deeper offline search by relaxing the selection criteria (e.g., requiring only one detection instead of two). The following steps describe our offline search. 


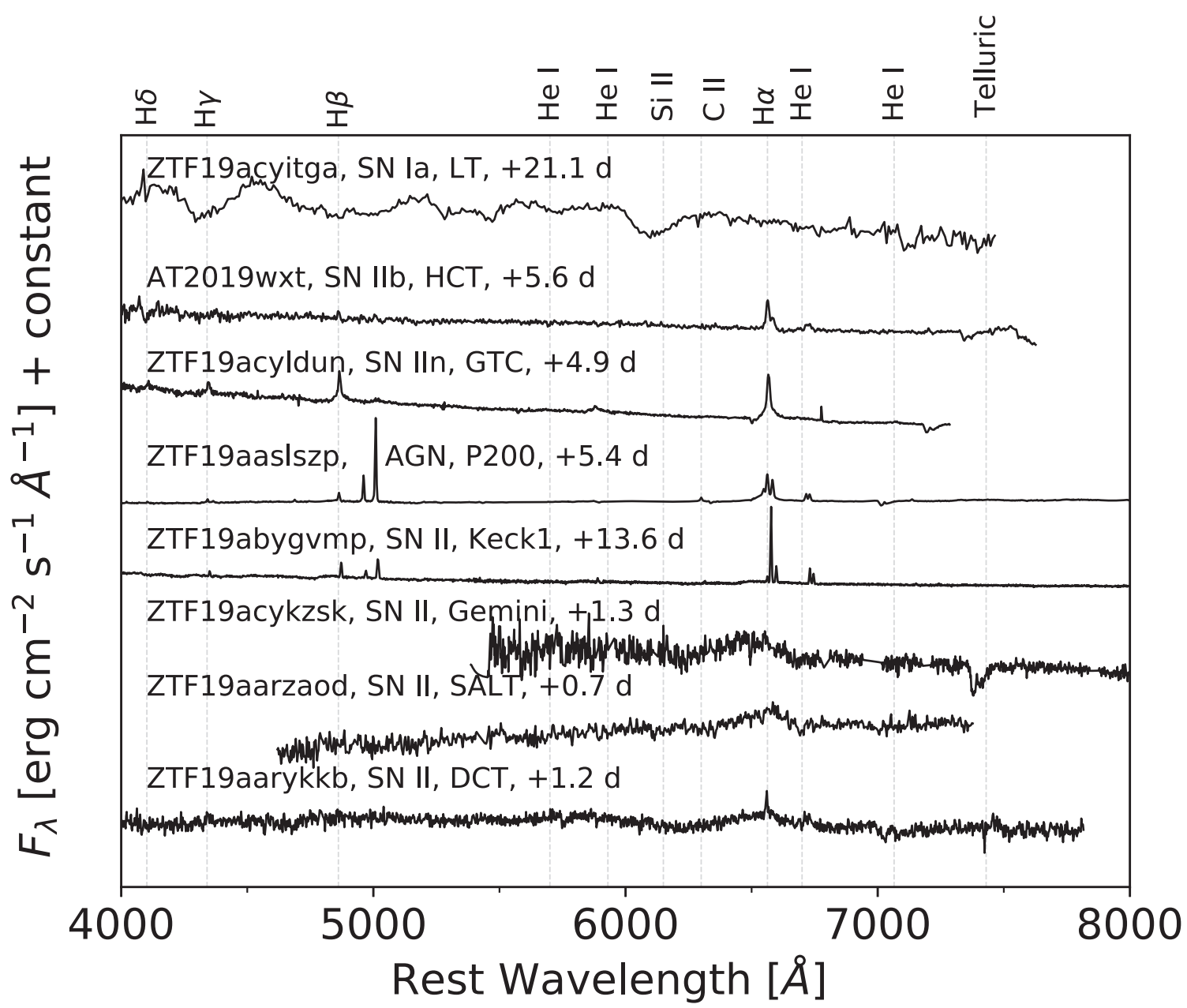

Figure 6. Collage of spectra taken during our GW follow-up in O3, one from each spectroscopic facility. The spectra displayed include six Type II SNe, one AGN, and one Type Ia SN and were taken with LT+SPRAT and GTC+OSIRIS in Roque de los Muchachos, Spain; P200+DBSP on Palomar Mountain, USA; Keck1 +LRIS and Gemini+GMOS-N on Maunakea, USA; SALT+RSS in Sutherland, South Africa; HCT+HFOSC in Hanle, India; and LDT+Deveny in Happy Jack, USA.

1. We used Kowalski to query the ZTF database looking for any source (i) located within $95 \%$ of the most updated sky map, (ii) never detected before the merger time, (iii) with at least one detection within $72 \mathrm{hr}$ of merger, (iv) with the last detection occurring within 10 days of the first detection, and (v) passing real/bogus thresholds of $\mathrm{rb}>0.5$ and drb $>0.8$ (or braai $>0.8$; Duev et al. 2019). Further details on the selection criteria will be described in I. Andreoni et al. (2020, in preparation).

2. Forced point-spread function (PSF) photometry was performed at the location of each transient candidate using ForcePhot, setting a detection threshold of signal-to-noise ratio $(\mathrm{S} / \mathrm{N})>3$, where the images were available.

3. The flux measured using forced photometry was stacked nightly in each band, allowing us to become sensitive to fainter sources when multiple images were available on the same night.

4. The rising and fading rates were computed in each band with a linear fit before and after the brightest data point of each light curve. A time baseline of $>3 \mathrm{hr}$ was required for the fit to be performed.

5. Candidates were selected with a fading rate more rapid than 0.3 mag day $^{-1}$ or rising rate faster than 1 mag day $^{-1}$.
We rejected candidates still detected after 6,12 , and 14 days after the merger time in the $g, r$, and $i$ bands, respectively. More details are given in I. Andreoni et al. (2020, in preparation).

The Kowalski query initially returned 8026 sources for the $13 \mathrm{GW}$ triggers. Applying all of the selection criteria described above, 453 candidates survived the automatic cuts. Of these, 21 had at least two ZTF alerts, and 432 had only one ZTF alert (additional detections were recovered by forced photometry and stacking).

Of the 21 sources with at least two detections in the ZTF alert stream, only five candidates passed visual inspection of the images and light curves: ZTF19acbxacj was an AGN candidate (Assef et al. 2018; Bailer-Jones et al. 2019); ZTF19abwsfsl was a cataloged CV (Gaia Collaboration 2018); ZTF19acbqtue was followed up with the Gemini Multi-Object Spectrograph (GMOS-N), and a quiescent source was found at $g=24.69 \pm 0.07 \mathrm{mag}$ with a color $g-i=1.89 \mathrm{mag}$, consistent with an M dwarf (West et al. 2011); ZTF19abyndjf was a fast-evolving transient without an obvious host galaxy; and ZTF19acbwtmt was hostless and had a previous detection in the PS1-DR2 catalog from 2012 (see Figure 8). For the last two candidates, upper limits between the GW merger time and the 


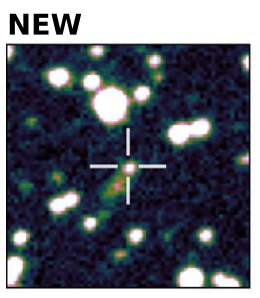

REF

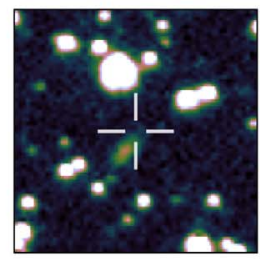

ZTF19aasmddt


NEW

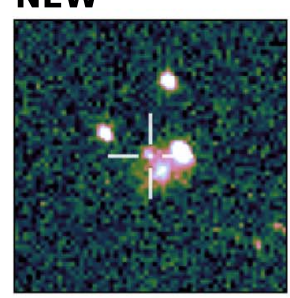

REF

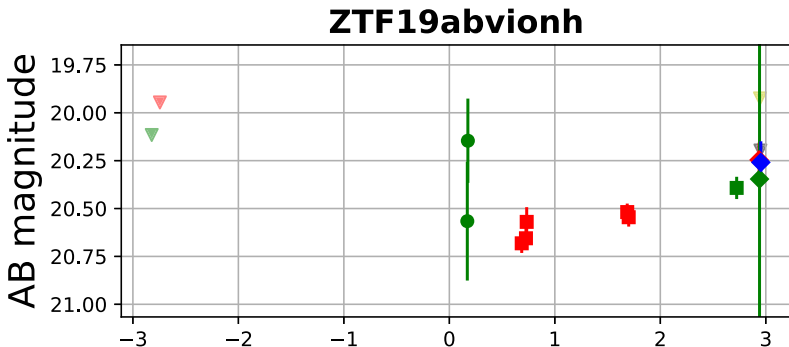

$\begin{array}{llll}u_{+} & -3 & -2 & -1 \\ + & & \text { Days after S190901ap }\end{array}$

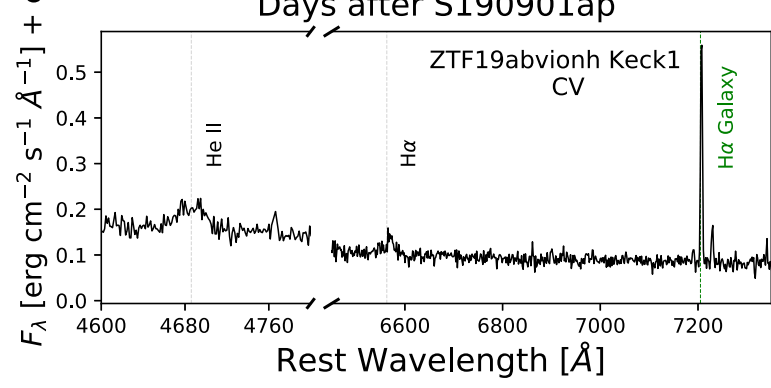

NEW

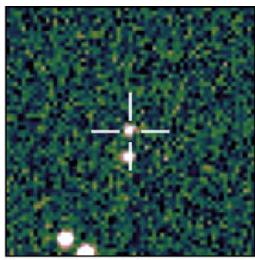

REF
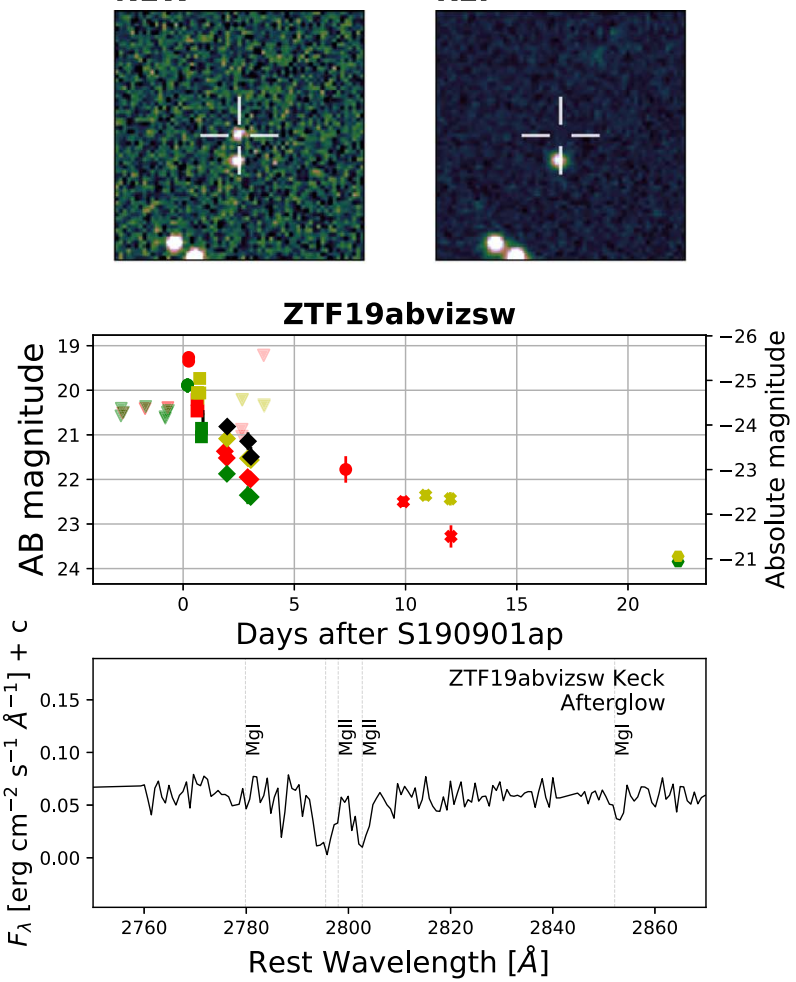

NEW

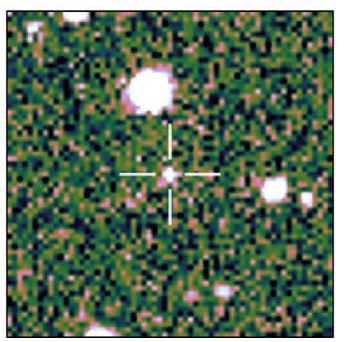

REF

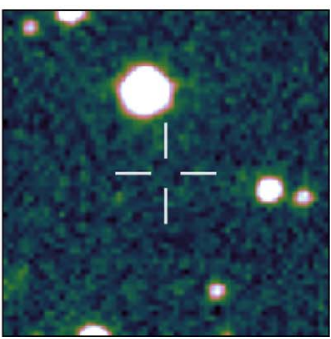

ZTF20aamvmzj



Figure 7. Collage of candidate counterparts found during real-time searches. We show a $7^{\prime \prime} \times 7^{\prime \prime}$ region with north up and east left for the discovery (NEW) and reference (REF) images. We also show the light curve of the candidate, where the $u$-, $g_{-}, r$-, $i$-, and $z$-band data are shown in blue, green, red, yellow, and black respectively. The ZTF data are presented with filled circles, while data from the LT, GIT, Keck, WHT, and LCO are presented as filled diamonds, squares, elongated diamonds, crosses, and pentagons, respectively. Absolute magnitude is shown for the candidates with a known redshift, and upper limits are shown as inverted triangles. We also display the spectra of the transient where available and mark the hydrogen and helium lines for ZTF19aasmddt (SN II), the H and He II features of ZTF19abvionh (CV), and the Mg I and Mg II lines for ZTF19abvizsw (long GRB afterglow). 
Table 2

List of Candidate Counterparts to S190426c

\begin{tabular}{|c|c|c|c|c|c|c|}
\hline Name & TNS & R.A. & Decl. & Host/Redshift & Discov. Mag & Rejection Crit. \\
\hline ZTF19aasmftm & AT2019sne & 325.9004479 & 77.8315634 & $0.156[s]$ & $g=18.78 \pm 0.19$ & SN Ia \\
\hline ZTF19aaslzjf & AT2019snh & 320.6262982 & 65.8134516 & $0.028[s]$ & $g=19.45 \pm 0.14$ & SN Ia \\
\hline ZTF19aasmddt & SN2019fht & 299.25055 & 9.7016748 & $0.028[s]$ & $g=18.6 \pm 0.11$ & SN II \\
\hline ZTF19aassfws & AT2019fuc & 298.6678611 & 61.2400121 & $\cdots$ & $r=21.35 \pm 0.21$ & Artifact \\
\hline ZTF19aaslszp & AT2019snj & 301.3434628 & 53.3990477 & $0.084[s]$ & $g=20.12 \pm 0.15$ & $\alpha_{r}=0.01, \mathrm{AGN}$ \\
\hline ZTF19aaslolf & AT2019snn & 288.7838539 & 79.4357187 & $\cdots$ & $r=21.12 \pm 0.18$ & $\alpha_{r}<0.01$, AGN, PS1 \\
\hline ZTF19aaslphi & AT2019sno & 297.3809977 & 61.9605925 & $\cdots$ & $r=21.26 \pm 0.20$ & $\alpha_{r}=-0.08$ \\
\hline ZTF19aaslpds & AT2019snq & 306.2625186 & 61.521461 & $\cdots$ & $r=19.9 \pm 0.14$ & $\alpha_{r}=0.03$ \\
\hline ZTF19aasmzqf & AT2019aaco & 353.5204911 & 78.9577781 & $\cdots$ & $r=19.86 \pm 0.09$ & $\alpha_{r}=0.01$ \\
\hline ZTF19aaslzfk & AT2019snd & 308.968271 & 72.3536353 & $\cdots$ & $g=20.0 \pm 0.26$ & $\alpha_{g}=-0.02$ \\
\hline ZTF19aaslvwn & AT2019snf & 299.059846 & 46.463559 & $\cdots$ & $g=20.68 \pm 0.17$ & $\alpha_{r}<0.01$ \\
\hline ZTF19aasmdir & AT2019sng & 300.2360007 & 9.504002 & $\cdots$ & $g=20.07 \pm 0.11$ & $\alpha_{r}<0.01$ \\
\hline
\end{tabular}

Table 3

List of Candidate Counterparts to S190901ap

\begin{tabular}{|c|c|c|c|c|c|c|}
\hline Name & TNS & R.A. & Decl. & Host/Redshift & Discov. Mag & Rejection Crit. \\
\hline ZTF19abvizsw & AT2019pim & 279.47282 & 61.497984 & $1.26[s]$ & $r=19.89 \pm 0.16$ & Long GRB afterglow \\
\hline ZTF19abwvals & AT2019pni & 73.250555 & 12.69303 & $0.091[s]$ & $r=18.96 \pm 0.30$ & SN Ia \\
\hline ZTF19abvixoy & AT2019pin & 279.552972 & 27.420935 & $\cdots$ & $r=18.93 \pm 0.10$ & $\alpha_{r}=0.23, \mathrm{CV}$ \\
\hline ZTF19abvionh & AT2019pip & 253.750924 & 14.05133 & $0.0985[s]$ & $g=20.57 \pm 0.31$ & $\alpha_{g}=0.10, \mathrm{CV}$ \\
\hline ZTF19abwsmmd & AT2019pnc & 22.666409 & -19.712405 & $0.0972[s]$ & $g=19.78 \pm 0.18$ & $\alpha_{g}=0.03$ \\
\hline ZTF19abvislp & AT2019pnx & 220.349708 & 54.151153 & $0.10[s]$ & $r=19.98 \pm 0.20$ & $\alpha_{r}=0.05$ \\
\hline ZTF19abxdvcs & AT2019qev & 252.010477 & 41.920087 & $\cdots$ & $g=20.64 \pm 0.28$ & $\alpha_{g}=0.03$ \\
\hline
\end{tabular}

Table 4

List of Candidate Counterparts to S190910d

\begin{tabular}{|c|c|c|c|c|c|c|}
\hline Name & TNS & R.A. & Decl. & Host/Redshift & Discov. Mag & Rejection Crit. \\
\hline ZTF19abyfhov & AT2019pvu & 260.693429 & 11.424436 & $0.13[s]$ & $g=19.92 \pm 0.22$ & SN Ia \\
\hline ZTF19abyfbii & AT2019pvz & 255.44162 & 11.602254 & $0.118[s]$ & $r=19.60 \pm 0.16$ & SN Ia 91T \\
\hline ZTF19abyfazm & AT2019pwa & 290.535876 & 48.069162 & $0.38[s]$ & $g=17.53 \pm 0.03$ & $\mathrm{CV}, \alpha_{r}=0.09$ \\
\hline ZTF19abyfhaq & AT2019pvv & 303.148593 & 49.392607 & $0[s]$ & $g=18.01 \pm 0.31$ & $\alpha_{r}=0.15$, Galactic \\
\hline
\end{tabular}

Table 5

List of Candidate Counterparts to S190910h

\begin{tabular}{|c|c|c|c|c|c|c|}
\hline Name & TNS & R.A. & Decl. & Host/Redshift & Discov. Mag & Rejection Crit. \\
\hline ZTF19abyheza & AT2019pxi & 332.913391 & 60.395816 & $0[s]$ & $r=16.14 \pm 0.13$ & $\mathrm{CV}, \alpha_{r}=0.08$ \\
\hline ZTF19abyhhml & AT2019pxj & 339.691635 & 55.936649 & $0[s]$ & $r=17.36 \pm 0.12$ & $\mathrm{CV}, \alpha_{r}=0.13$ \\
\hline ZTF19abyirj1 & AT2019pxe & 30.471176 & 30.73355 & $0.1[s]$ & $r=19.45 \pm 0.13$ & SN Ia \\
\hline ZTF19abyjcom & AT2019pxk & 32.936353 & 12.033344 & $\ldots$ & $r=19.63 \pm 0.24$ & Artifact \\
\hline ZTF19abyjcon & AT2019pxl & 33.252469 & 12.472604 & $\ldots$ & $r=19.87 \pm 0.19$ & Artifact \\
\hline ZTF19abyjcoo & AT2019pxm & 33.089712 & 12.297698 & $<0.03[p]$ & $r=19.95 \pm 0.24$ & $\alpha_{r}=0.06$ \\
\hline ZTF19abyjfiw & AT2019pxn & 39.186807 & 34.647299 & $\cdots$ & $g=20.13 \pm 0.21$ & $\alpha_{r}<0.01$ \\
\hline ZTF19abygvmp & AT2019pzg & 28.976258 & 41.090979 & $0.049[s]$ & $r=20.13 \pm 0.25$ & SN II \\
\hline ZTF19abyiwiw & AT2019pzi & 340.521441 & 55.220244 & ... & $r=18.58 \pm 0.30$ & $\alpha_{g}=0.20$ \\
\hline ZTF19abylleu & AT2019pyu & 355.338225 & -23.450706 & $\cdots$ & $r=19.19 \pm 0.24$ & $\alpha_{g}=0.03$ \\
\hline ZTF19abymhyi & AT2019pzh & 340.85572 & 34.186344 & $<0.03[p]$ & $g=20.36 \pm 0.23$ & $\alpha_{g}=-0.13$ \\
\hline
\end{tabular}

Table 6

List of Candidate Counterparts to S190923y

\begin{tabular}{lccccc}
\hline \hline Name & TNS & R.A. & Decl. & Host/Redshift & Discov. Mag \\
\hline ZTF19acbmopl & AT2019rob & 114.040207 & 28.487381 & $<0.03[p]$ & $g=19.64 \pm 0.27$ \\
\hline
\end{tabular}


Table 7

List of Candidate Counterparts to S190930t

\begin{tabular}{lcccccc}
\hline \hline Name & TNS & R.A. & Decl. & Host/Redshift & Discov. Mag & Rejection Crit. \\
\hline ZTF19acbpqlh & AT2019rpn & 319.9216636 & 37.5220721 & $0.026[s]$ & $g=19.47 \pm 0.18$ & SN II \\
ZTF19acbwaah & AT2019rpp & 162.3277489 & 22.9827302 & $0.031[s]$ & $17.61 \pm 0.08$ & SN Ia \\
ATLAS19wyn & AT2019rpj & 339.8367397 & 31.4916262 & $0.0297[s]$ & $g=19.32 \pm 0.11$ & SN II \\
\hline
\end{tabular}

Table 8

List of Candidate Counterparts to S191205ah

\begin{tabular}{|c|c|c|c|c|c|c|}
\hline Name & TNS & R.A. & Decl. & Host/Redshift & Discov. Mag & Rejection Crit \\
\hline ZTF19acxpnvd & AT2019wkv & 175.361851 & 8.241201 & $<0.03[p]$ & $i=19.58 \pm 0.20$ & $\alpha_{g}=0.06$ \\
\hline ZTF19acxoywk & AT2019wix & 149.896148 & 13.915051 & $0.05[s]$ & $r=19.69 \pm 0.21$ & $\alpha_{g}=-0.15$ \\
\hline ZTF19acxoyra & AT2019wid & 153.093775 & 8.609330 & $0.09[s]$ & $r=19.14 \pm 0.19$ & $\alpha_{g}=0.05$ \\
\hline ZTF19acxpwlh & AT2019wiy & 155.712970 & 23.603273 & $<0.24[p]$ & $g=19.77 \pm 0.19$ & $\alpha_{r}=0.07$ \\
\hline ZTF19acyiflj & AT2019wmy & 152.899874 & 23.943843 & $0.081[s]$ & $r=20.05 \pm 19.63$ & SN Ia \\
\hline ZTF19acxowrr & AT2019wib & 154.871458 & 27.883738 & $0.05[s]$ & $r=19.00 \pm 0.13$ & SN II \\
\hline ZTF19acyitga & AT2019wmn & 159.796830 & 5.161942 & $0.071[s]$ & $r=19.20 \pm 0.16$ & SN Ia \\
\hline
\end{tabular}

Table 9

List of Candidate Counterparts to S191213g Reported in GCN 26424 and 26437

\begin{tabular}{|c|c|c|c|c|c|c|}
\hline Name & TNS & R.A. & Decl. & Host/Redshift & Discov. Mag & Rejection Crit. \\
\hline ZTF19acykzsk & SN2019wqj & 32.904547 & 34.041346 & $0.021[s]$ & $g=19.0 \pm 0.06$ & SN II \\
\hline ZTF19acymaru & AT2019wnh & 80.461954 & -19.266401 & $0.167[s]$ & $r=19.92 \pm 0.16$ & SN Ia \\
\hline ZTF19acykzsp & AT2019wne & 28.359144 & 31.801012 & $0.16[s]$ & $r=20.08 \pm 0.31$ & SN Ia \\
\hline ZTF19acyfoha & AT2019wkl & 85.104365 & -18.097630 & $0.04[s]$ & $g=17.31 \pm 0.08$ & SN Ia \\
\hline ZTF19acymcwv & AT2019wni & 36.248920 & 47.497844 & $0.09[s]$ & $r=19.76 \pm 0.24$ & SN Ia \\
\hline ZTF19acykwsd & AT2019wnl & 33.088072 & 41.388708 & $\cdots$ & $r=19.3 \pm 0.25$ & Artifact \\
\hline ZTF19acylvus & AT2019wnk & 83.631136 & -19.420244 & $0.104[s]$ & $r=19.45 \pm 0.24$ & SN Ia \\
\hline ZTF19acymcna & AT2019wnn & 33.207899 & 40.999726 & $0.138[s]$ & $r=20.48 \pm 0.22$ & $\alpha_{r}=-0.01, \mathrm{AGN}$ \\
\hline ZTF19acykyqu & AT2019wre & 38.819646 & 38.319851 & $\ldots$ & $g=20.94 \pm 0.21$ & Stellar-PS1-DR2 \\
\hline ZTF19acykyrz & AT2019wrf & 36.064972 & 38.080388 & $\cdots$ & $g=20.83 \pm 0.17$ & Stellar-PS1-DR2 \\
\hline ZTF19acykyzj & AT2019wrg & 36.056624 & 51.367126 & $\cdots$ & $g=19.75 \pm 0.20$ & $\alpha_{r}=-0.03$ \\
\hline ZTF19acykzfy & AT2019wrh & 43.115194 & 41.660303 & $\cdots$ & $g=20.34 \pm 0.20$ & Stellar-PS1-DR2 \\
\hline ZTF19acyldum & AT2019wrn & 79.681883 & -7.185279 & $\cdots$ & $g=19.41 \pm 0.13$ & PS1-DR2 detection \\
\hline ZTF19acyldun & AT2019wrt & 79.199993 & -7.478682 & $0.057[s]$ & $g=19.42 \pm 0.17$ & $\alpha_{r}=0.09, \mathrm{LBV}$ \\
\hline ZTF19acymapa & AT2019wro & 78.207321 & -5.948936 & $\ldots$ & $g=18.54 \pm 0.22$ & $\alpha_{r}^{\ddagger}=-0.06$ \\
\hline ZTF19acymaxu & AT2019wrp & 82.952485 & -26.694523 & $<0.13[p]$ & $r=18.65 \pm 0.06$ & $\alpha_{r}=0.03$ \\
\hline ZTF19acymixu & AT2019wrr & 90.913936 & 60.728245 & $0.14[s]$ & $r=19.66 \pm 0.32$ & SN Ia \\
\hline ZTF19acymlhi & AT2019wrs & 91.592426 & -18.804727 & $\ldots$ & $r=17.99 \pm 0.26$ & $\alpha_{r}^{\ddagger}=-0.17$ \\
\hline
\end{tabular}

Note. The candidates for which photometric evolution has been calculated with a baseline $(\Delta t)$ between 2 and 3 days are marked with $\$$.

first transient detection disfavor their multimessenger association with S190930t or S190910d, respectively (see Figure 8).

Of the 432 sources with only one detection in the ZTF alert stream, only nine candidates passed the visual inspection of the images and light curves. Most other candidates were ruled out as stellar flares, image subtraction artifacts, asteroids, or sporadic nuclear variability. Of these nine candidates, six had photometric or spectroscopic redshifts of the host galaxy too far to be consistent with the GW distance. All three remaining candidates were found during follow-up of S190901ap: ZTF19abvpeir, ZTF19abvozxv, and ZTF19abvphxm. All three are likely SNe or AGNs, given that their absolute magnitudes at the distance of their putative hosts are between -18.0 and $-18.8 \mathrm{mag}$ and their locations are consistent with the Galaxy nuclei. We show some light curves and host galaxies in Figure 8.

In summary, all candidates were ruled out as possible kilonovae in both the real-time and offline analyses.

\section{Discussion}

We start by treating all triggers as bona fide astrophysical events regardless of FAR, assuming that kilonovae accompanying BNS and NSBH mergers are drawn from a common population, and analyzing the implications of zero kilonova detections. Later, we relax these assumptions. Since kilonova models have a wide range of estimates depending on several intrinsic and extrinsic parameters (e.g., ejecta mass, ejecta velocity, lanthanide fraction, viewing angle, remnant lifetime), we took a model-independent approach toward constraining the luminosity function.

Serving as our benchmark is GW170817. The ZTF observations were taken as $g$ - and $r$-band pairs, and GW170817 was discovered at an $i$-band magnitude of 17.3 mag about $10.8 \mathrm{hr}$ after merger (Coulter et al. 2017). Compiling and fitting all published data in the $g$ and $r$ bands for GW170817 in the first 3 days after merger (Andreoni et al. 2017; Arcavi et al. 2017; Coulter et al. 2017; Cowperthwaite et al. 2017; Díaz et al. 2017; 

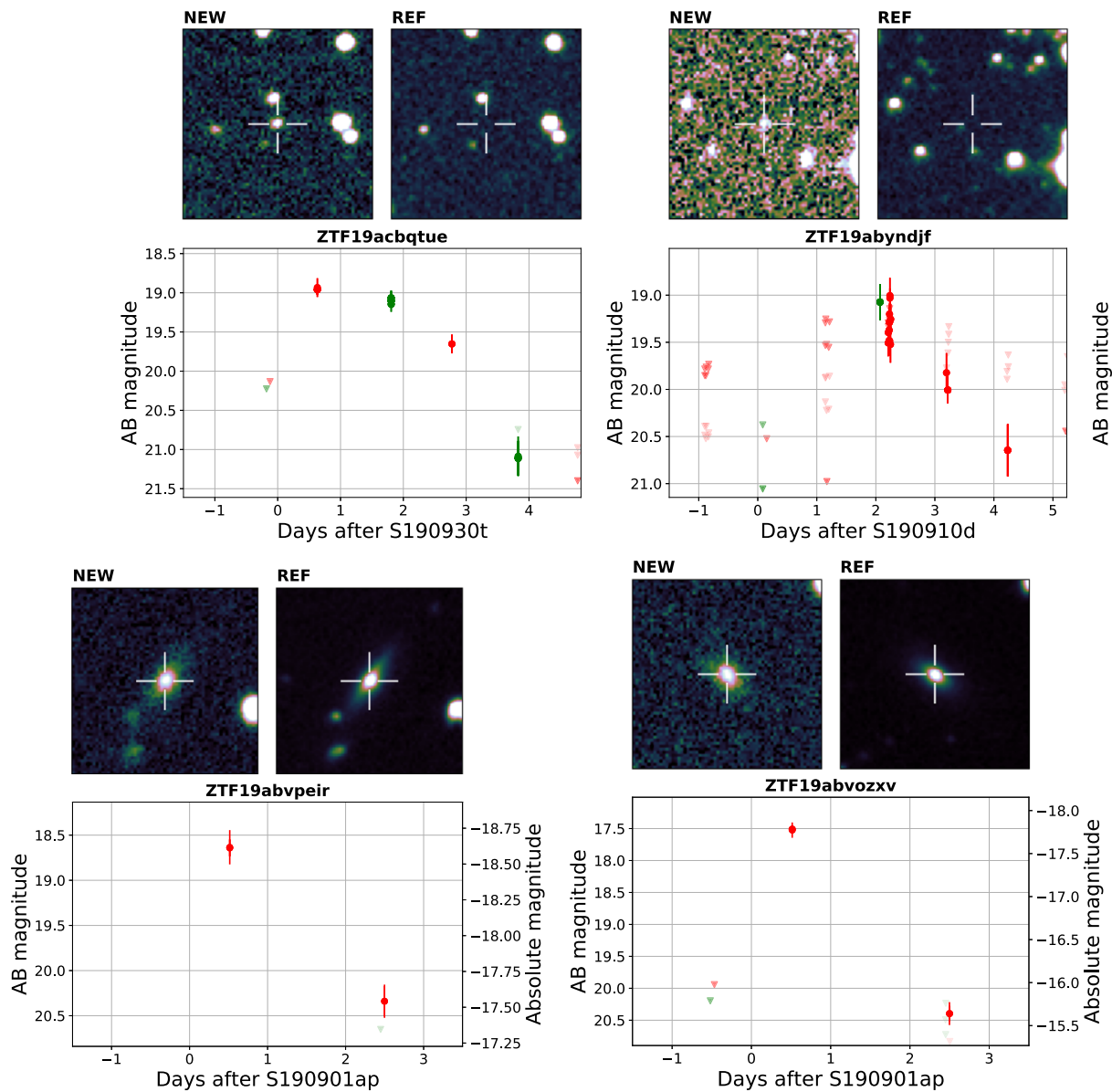


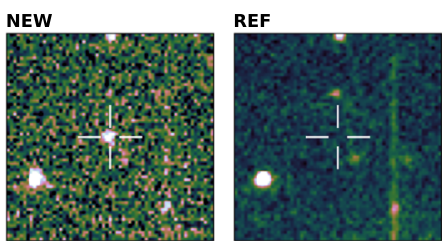

ZTF19acbwtmt

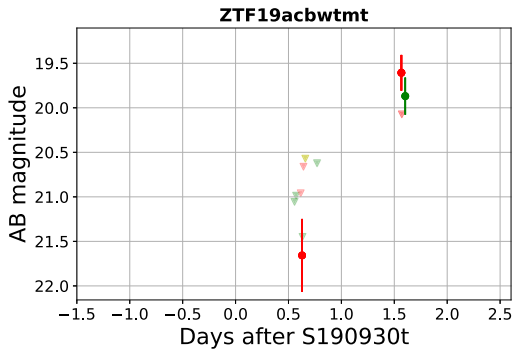

NEW
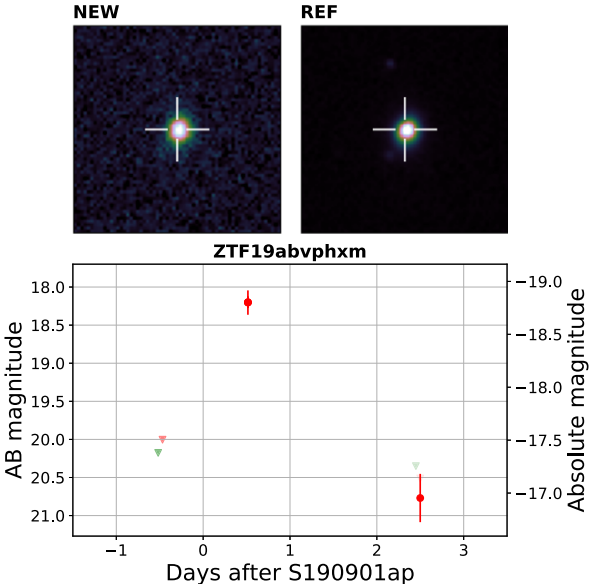

Figure 8. Collage of candidate counterparts found in deeper offline searches. Each candidate in the top row has two or more ZTF alerts: ZTF19acbqtue was ruled out, as we found a quiescent stellar source with GMOS-N; ZTF19abyndjf does not have a galaxy in its vicinity; and ZTF19acbwtmt had archival activity in PS1-DR2. Each candidate in the bottom row had only one ZTF alert but was flagged as interesting after performing forced photometry. These three candidates are nuclear transients that are ruled out, as their absolute magnitudes are brighter than what is expected for kilonovae.

Table 10

List of Candidate Counterparts to S200213t

\begin{tabular}{|c|c|c|c|c|c|c|}
\hline Name & TNS & R.A. & Decl. & Host/Redshift & Discov. Mag & Rejection Crit. \\
\hline ZTF20aamvqxl & AT2020ciy & 29.237921 & 53.668882 & $0.102[s]$ & $g=19.44 \pm 0.17$ & SN Ia \\
\hline ZTF20aamvnth & AT2020cjb & 18.337721 & 49.645539 & $0.061[s]$ & $g=19.95 \pm 0.17$ & SN II \\
\hline ZTF20aamvoxx & AT2020cjg & 39.399095 & 26.920616 & $0.097[s]$ & $g=19.47 \pm 0.12$ & SN Ia \\
\hline ZTF20aamvtip & AT2020cje & 38.082538 & 27.810094 & $0.151[s]$ & $g=20.3 \pm 0.16$ & SN Ia \\
\hline ZTF20aamvnat & AT2020ciz & 27.239552 & 56.354579 & $0.0[s]$ & $g=17.42 \pm 0.05$ & $\mathrm{CV}$ \\
\hline ZTF20aamvmzj & AT2020cja & 27.189195 & 51.430481 & $\cdots$ & $g=19.46 \pm 0.11$ & $\alpha_{r}=0.04$ \\
\hline ZTF20aamvoeh & AT2020cjc & 33.502011 & 38.936317 & $0.14[s]$ & $g=20.25 \pm 0.12$ & SN Ia \\
\hline ZTF20aamvodd & AT2020cjf & 37.482387 & 50.319427 & $0.0[s]$ & $g=18.92 \pm 0.11$ & Stellar flare \\
\hline ZTF20aanakwb & AT2020cls & 6.5215391 & 42.7737224 & $\cdots$ & $g=20.75 \pm 0.27$ & Stellar \\
\hline ZTF20aanaltd & AT2020clt & 9.7406716 & 43.4410695 & $0.2[s]$ & $g=20.57 \pm 0.23$ & SN Ia \\
\hline ZTF20aanaksk & AT2020clu & 19.4356399 & 31.1744954 & $<0.03[p]$ & $g=20.27 \pm 0.10$ & PS1 \\
\hline ZTF20aanallx & AT2020clv & 6.3666608 & 51.2233877 & $\ldots$ & $g=20.58 \pm 0.28$ & Outside the LALInfernce map \\
\hline ZTF20aanaoyz & AT2020clw & 24.5940995 & 23.3822569 & $0.276[s]$ & $g=21.28 \pm 0.27$ & SN Ia \\
\hline ZTF20aamvpvx & AT2020clx & 31.9402981 & 20.0306147 & $0.074[s]$ & $g=19.95 \pm 0.14$ & SN II \\
\hline ZTF20aanamcs & AT2020crc & 13.74333345 & 43.4980245 & $0.093[s]$ & $g=20.98 \pm 0.28$ & SN II \\
\hline ZTF20aanakge & AT2020crd & 12.6306233 & 41.484178 & $0.1272[s]$ & $g=20.38 \pm 0.33$ & SN Ia \\
\hline ZTF20aanaqhe & AT2020cre & 17.0425796 & 45.5256583 & $\ldots$ & $g=20.63 \pm 0.27$ & $\alpha_{g}=-0.08$ \\
\hline ZTF20aanakes & AT2020cly & 2.0985443 & 38.0441264 & $\ldots$ & $g=20.79 \pm 0.21$ & PS1 \\
\hline ZTF20aanakcd & AT2020cmr & 8.1571223 & 41.3156371 & $0.077[s]$ & $g=20.48 \pm 0.17$ & SN IIn \\
\hline
\end{tabular}

Drout et al. 2017; Evans et al. 2017; Pian et al. 2017; Smartt et al. 2017; Utsumi et al. 2017; Valenti et al. 2017; Pozanenko et al. 2018), we find that GW 170817 had a decline rate of
0.9 mag day $^{-1}$ in the $r$ band and 1.3 mag day $^{-1}$ in the $g$ band. Extrapolating this decline rate to merger time and correcting for line-of-sight extinction, GW170817 may have peaked at 


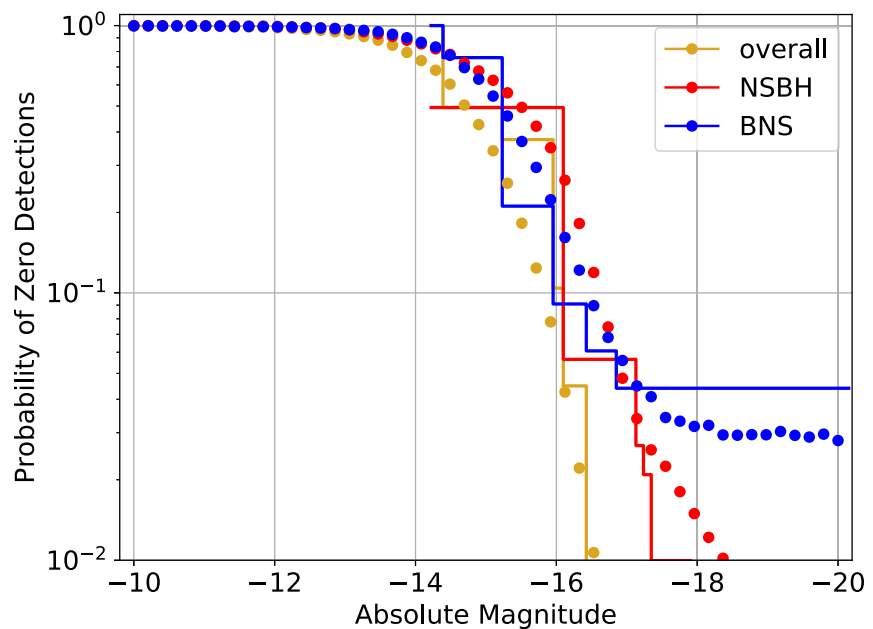

Figure 9. Joint probability of zero detections as a function of absolute magnitude of the kilonova after correcting for line-of-sight extinction. Solid lines represent rough estimates from median estimates. Filled circles represent estimates that take into account the spatial variation in depth, GW distance, and GW probability.

$-16.54 \mathrm{mag}$ in the $r$ band and $-16.69 \mathrm{mag}$ in the $g$ band (we caution that some kilonova models predict a finite rise time). Here we choose to compare the ZTF limits to an average of these two filters, i.e., -16.6 mag at peak and a decline rate of 1 mag day $^{-1}$.

\subsection{Joint Probability of Zero Detections}

We estimate the joint probability of zero kilonova detections as a product of $\left(1-q_{i}\right)$ terms, where $q_{i}$ is the enclosed probability for each event as listed in Table 1 (see the sixth column; we used LALInference probability where available). If we were sufficiently sensitive to finding kilonovae in all $13 \mathrm{GW}$ events, the joint probability of zero detections would be only $0.017 \%$. However, each merger had a different observed depth, observed cadence, and GW distance estimate and thus a different sensitivity to detecting kilonovae.

First, we use the median image depth for each trigger and the median GW distance to each trigger to compute a median absolute magnitude sensitivity limit. We correct the median absolute magnitude for the median extinction along the line of sight. In Figure 9, in each luminosity bin, we compute the joint nondetection probability only for the subset of events for which the ZTF observations were sufficiently sensitive. We find that ZTF follow-up of four (six) GW events had a sensitivity deeper than $-16.0 \mathrm{mag}(-16.6 \mathrm{mag})$, and the joint nondetection probability is only $4.5 \%(0.34 \%)$. Moreover, three of the four (four of the six) had a preliminary BNS classification, and for all three, the ZTF follow-up began within $4 \mathrm{hr}$ of merger (see Table 1).

Second, we use injection and recovery of fake sources to better quantify both the degree of variation in the depths of individual exposures and the spatial variation in the GW distance estimates. We use an open-source tool called simsurvey ${ }^{75}$ (Feindt et al. 2019). As input, the tool takes a list of ZTF pointings (observation time, R.A., decl., limiting magnitude, filters, processing success of each CCD quadrant). We inject 10,000 sources distributed according to the 3D GW sky map probability distribution in each luminosity bin (50 bins between -10 and $-20 \mathrm{mag}$ ). Initially, we assume that each

\footnotetext{
75 https://github.com/ZwickyTransientFacility/simsurvey
}

kilonova stays at a constant luminosity between the merger time and 3 days after merger. We require a single observation at the necessary depth for recovery. In addition to losing sources in unobserved fields, we lose sources that land in ZTF chip gaps, chips that failed processing, or chips that were less sensitive due to higher line-of-sight Galactic extinction. This tool does not take into account any detections that would be lost to inefficiency in the software pipeline.

The recovery fraction for each event is shown in Figure 10. We convert this to a joint nondetection probability estimate by multiplying $\left(1-{ }_{p i}\right)$ in each luminosity bin and overlay this as discrete points on the median estimates above in Figure 9. We find consistent results; the joint probability of zero detections at $-16.1 \mathrm{mag}(-16.6 \mathrm{mag})$ is only $4.2 \%(0.8 \%)$. If we separate the NSBH and BNS populations, the joint nondetection probability at $-16.6 \mathrm{mag}$ is $9.7 \%$ for $\mathrm{NSBH}$ and $7.9 \%$ for BNS. This is not surprising, as the BNS triggers were, on average, closer than the NSBH triggers. We note that this application of simsurvey is different compared to previous applications for SN rates, which were uniform in volume. Taking into account the exact 3D GW sky map is more accurately representative of our success in searching for the counterpart to a GW source on an event-by-event basis.

Third, in addition to spatial variations in depth and distance, we take into account the possible time variations in the light curves of kilonovae (Figure 11). The time window for our observations is limited to within 3 days of merger. We relax the constant luminosity assumption above and inject kilonovae into simsurvey that fade linearly between zero and 1 mag day ${ }^{-1}$. In Figure 11, we color-code the recovery efficiency for a given peak luminosity and photometric decay rate in any filter $(g$ or $r$ band). Any slice of this plot can be converted to a joint probability of zero detections as a function of absolute magnitude. We compare to the GW170817 benchmark of an extrapolated peak of $-16.6 \mathrm{mag} \mathrm{day}^{-1}$ and a fade rate of $1 \mathrm{mag}$ day $^{-1}$. We find a joint probability of zero detections of $7 \%$.

Fourth, in addition to spatial and time variations, we inject kilonova models into simsurvey and calculate the recovery fraction. We use the best fit to GW170817 from the kilonova model grid in Dietrich et al. (2020) computed using the radiative transfer code POSS IS (Bulla 2019). This model fit assumes a rise time, color evolution, and viewing angle of GW170817. The joint nondetection probability is $30 \%$. Even if all kilonova ejecta parameters were similar to GW170817, the viewing angle would be different for different events. Assuming random viewing angles drawn from a distribution uniform in $\cos (\theta)$, we inject a model grid and find that the joint nondetection probability is $49 \%$. We caution that the model used here underestimates the early $g$-band flux of GW170817 by $0.3 \mathrm{mag}$; thus, the recovery fraction estimated here could also be underestimated.

\subsection{Constraining the Kilonova Luminosity Function}

Next, we consider the implications of the zero-detection probability function on the underlying luminosity function. Let us say the luminosity function dictates that a fraction $f_{b}$ of kilonovae are brighter than a given absolute magnitude. Then,

$$
(1-\mathrm{CL})=\prod_{i=1}^{N}\left(1-f_{b}^{*} p_{i}\right),
$$

where CL is the confidence level and ${ }_{p i}$ is the event-by-event probability of detection. At a given absolute magnitude, we 

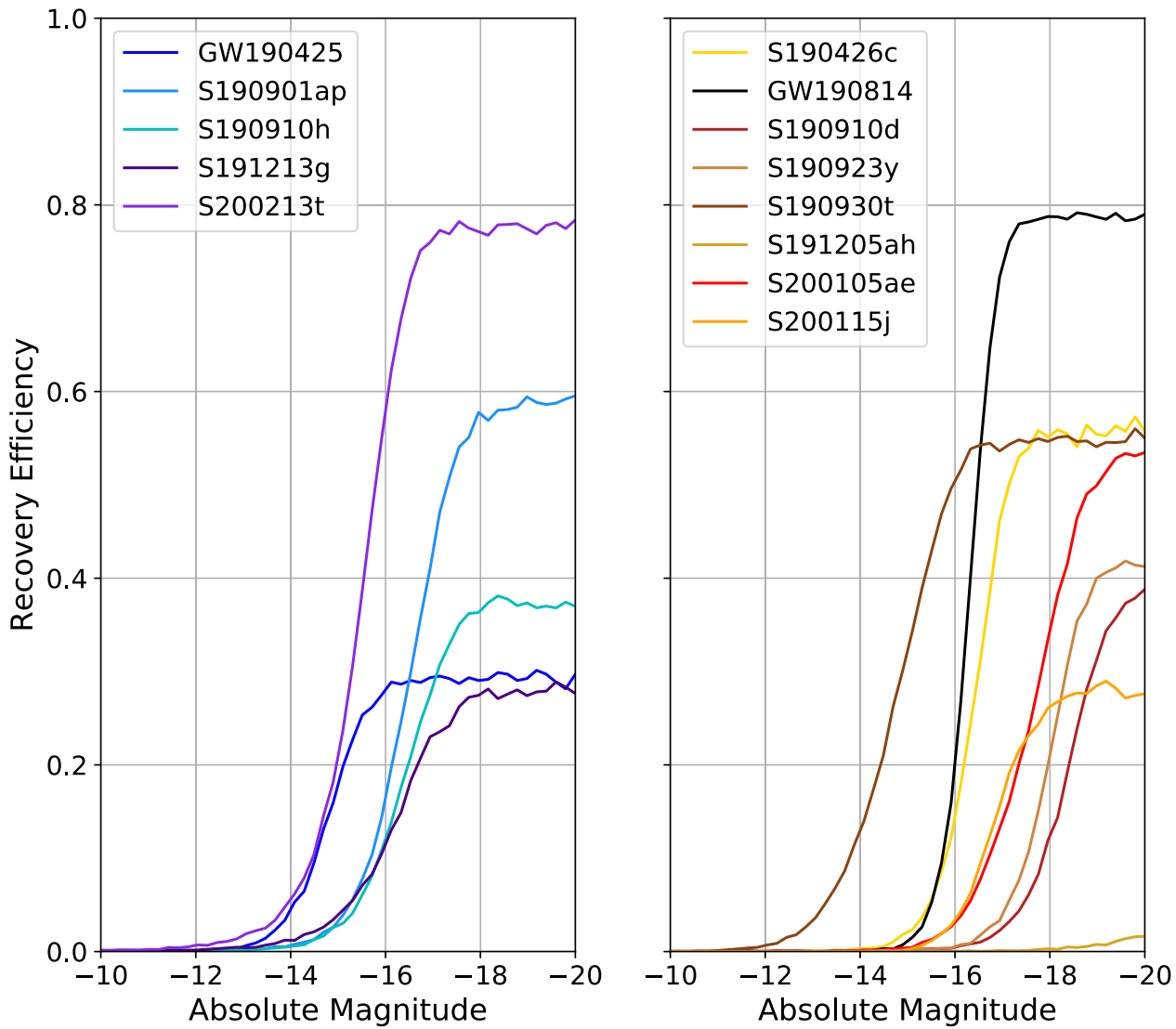

Figure 10. Event-by-event recovery efficiency using simsurvey as a function of absolute magnitude for BNS (left panel) and NSBH (right panel) mergers. The recovery efficiency corresponds to the number of kilonovae detected divided by the total number of kilonovae injected. Kilonovae were injected according to the 3D probability distribution of the sky map.

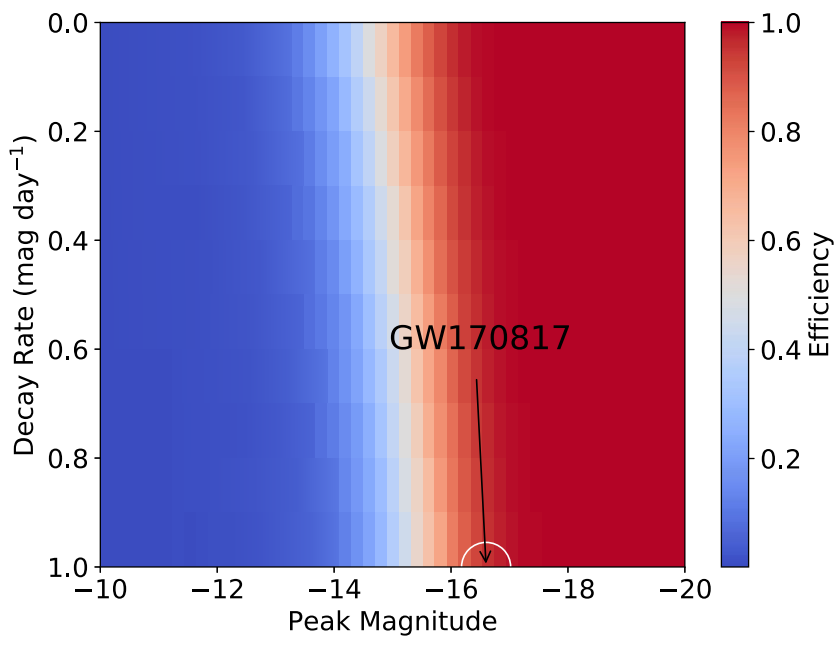

Figure 11. Composite efficiency map using simsurvey assuming a linear model for the kilonova with a peak absolute magnitude and fixed decay rate. The colorcoding shows the recovery efficiency, or the number of recovered kilonovae within observed regions divided by the total number of kilonovae injected in the sky map. Based on an analysis of a compilation of data from GW170817 (Andreoni et al. 2017; Coulter et al. 2017; Cowperthwaite et al. 2017; Díaz et al. 2017; Drout et al. 2017; Evans et al. 2017; Pian et al. 2017; Smartt et al. 2017; Utsumi et al. 2017; Valenti et al. 2017; Arcavi 2018; Pozanenko et al. 2018), we compute an average extrapolated peak magnitude of $\sim-16.6$ and a decay rate of $\sim 1$ mag day $^{-1}$. If all kilonovae are like GW170817, the joint probability of zero detections is $7 \%$.

compute $_{p i}$ as the recovery fraction from the simsurvey injections for the fading and flat light-curve evolution estimates discussed above that take into account the spatial variation in distance, depth, and enclosed probability. Solving for $f_{b}$ at a $90 \%$ confidence level, we plot our results in Figure 12 . At the bright end, we find that no more than $\approx 40 \%$ of kilonovae can be brighter than $-18 \mathrm{mag}$. At the faint end, our observations place no constraints on the luminosity function fainter than $-15.5 \mathrm{mag}$. The luminosity of GW170817 at the merger time is unknown, and various models predict diverse rates of evolution in that first day after merger. As discussed above, we use an extrapolated peak of $-16.6 \mathrm{mag}$ and a fade rate of $1 \mathrm{mag} \mathrm{day}^{-1}$ for GW170817 as a benchmark. We find that no more than $<57 \%(<89 \%)$ of kilonovae could be brighter than $-16.6 \mathrm{mag}$ for the flat (fading) light-curve assumptions.

The GW triggers had a very wide range of FARs. Weighting by the available low-latency values for the terrestrial probability $\left(t_{i}\right)$, we fold this into our luminosity function constraint as

$$
(1-\mathrm{CL})=\prod_{i=1}^{N}\left(1-f_{b}^{*} p_{i}^{*}\left(1-t_{i}\right)\right) \text {. }
$$

In Figure 12, we show that the resulting constraints on $f_{b}$ (red line) are worse only by a difference of $\approx 10 \%$.

Next, we investigate the implications of this constraint on the kilonova parameter space. There are no theoretical luminosity functions available in the literature that we can directly compare to. A model grid is available (Kasen et al. 2017) as a function of three parameters: ejecta mass $M_{\mathrm{ej}}$, ejecta velocity $v_{\mathrm{ej}}$, and 




Peak Absolute Magnitude


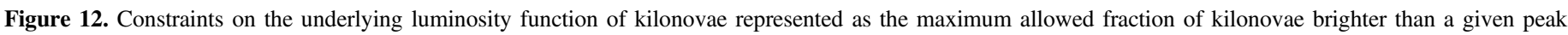



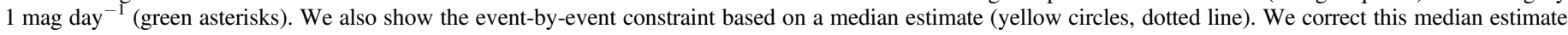

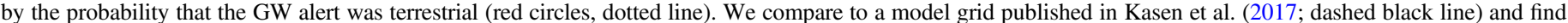

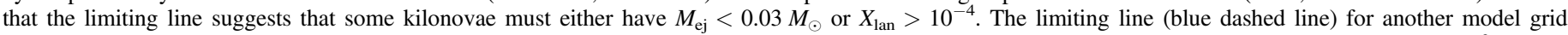
(Bulla 2019; Dietrich et al. 2020) suggests that some kilonovae must be fainter than GW170817 with $M_{\mathrm{ej}, \mathrm{dyn}}<0.005 M_{\odot}, M_{\mathrm{ej}, \mathrm{pm}}<0.05 M_{\odot}$, or $\phi>30^{\circ}$.

lanthanide fraction $X_{\text {lan }}$. The best-fit model to GW170817 from Kasen et al. (2017) suggested two components: a blue kilonova $\left(0.025 M_{\odot}, 0.3 c, 10^{-4.5}\right)$ and a red kilonova $\left(0.04 M_{\odot}, 0.1 c\right.$, $10^{-2}$ ). The blue component dominates at an early time and is more relevant to the ZTF searches described in this paper. Comparing to our luminosity function constraints, we find that our limits suggest that a wide range of parameters are allowed, e.g., $M_{\mathrm{ej}}=[0.03,0.1] M_{\odot}, \quad v_{\mathrm{ej}}=[0.05,0.3] c$, and $X_{\mathrm{lan}}=\left[10^{-5}\right.$, $\left.10^{-4}\right]$; stricter distributions that yield a brighter kilonova population (e.g., higher ejecta mass or lower lanthanide fraction) are not allowed. Thus, some kilonovae must have $M_{\text {ej }} \leqslant 0.03 M_{\odot}$ or $X_{\text {lan }}>10^{-4}$ to be consistent with the ZTF constraints.

Similarly, we compare our luminosity function constraints to the kilonova grid from Dietrich et al. (2020) computed using the radiative transfer code POSSIS (Bulla 2019). In addition to the observer viewing angle, this grid depends on three parameters: the dynamical ejecta mass $\left(M_{\mathrm{ej}, \mathrm{dyn}}\right)$, the postmerger wind ejecta mass $\left(M_{\mathrm{ej}, \mathrm{pm}}\right)$, and the half-opening angle of the lanthanide-rich ejecta component $(\phi)$. A model with $M_{\text {ej,dyn }}=0.005 M_{\odot}$, $M_{\text {ej,pm }}=0.05 M_{\odot}$, and $\phi=30^{\circ}$ provides a good fit to GW170817 (see Figure 8 of Dietrich et al. 2020). As shown in Figure 12, our constraints suggest that some kilonovae must be fainter than GW170817, i.e., must have either $M_{\text {ej,dyn }}<$ $0.005 M_{\odot}, M_{\mathrm{ej}, \mathrm{pm}}<0.05 M_{\odot}$, or $\phi>30^{\circ}$.

\section{Conclusions and Way Forward}

In summary, the ZTF coverage (excluding weather-impacted S191205ah) spanned enclosed probabilities from $22 \%$ to $89 \%$, median depths from 20.1 to $21.5 \mathrm{mag}$, and time lags between merger and the start of observations from $11 \mathrm{~s}$ to $13.7 \mathrm{hr}$. The follow-up by the GROWTH team comprised 340 UltraViolet/ Optical/InfraRed (UVOIR) photometric points, 64 OIR spectra, and three radio images. Additionally, many other teams also followed up ZTF candidates. Thanks to the extensive follow-up, all candidate counterparts were ruled out.

The GW triggers had localization areas ranging from 23 to $24,264 \mathrm{deg}^{2}$, distances from 108 to $632 \mathrm{Mpc}$, and FARs from 1.5 to $10^{-25} \mathrm{yr}^{-1}$. Assuming that all $\mathrm{GW}$ alerts were astrophysical, we conclude that the joint probability of zero detections is only $4.2 \%$ if all kilonovae are at least as bright as GW170817 at discovery. Furthermore, assuming kilonovae from BNS and NSBH mergers are drawn from a common population, we find that no more than $<57 \%(<89 \%)$ of kilonovae could be brighter than -16.6 mag for the flat (fading by $1 \mathrm{mag} \mathrm{day}^{-1}$ ) assumptions respectively at $90 \%$ confidence.

The median time lag of the ZTF observations in $\mathrm{O} 3$ was only $1.5 \mathrm{hr}$ after merger. This further constrains the unknown, earlytime emission of kilonovae in the $g$ and $r$ bands. Some models predict that the early emission could be very hot and bright in 
the UV; this can only be addressed once wide-field UV imagers (e.g., Dorado, ULTRASAT, and DUET) are launched.

Given the expected differences in sensitivity between the LIGO and Virgo interferometers, events in $\mathrm{O} 4$ are likely to be similarly coarsely localized (until KAGRA or LIGO India come online with high sensitivity). Moreover, given the increased GW sensitivity, we expect more events that are further away. Thus, we plan to implement stricter selection criteria. Specifically, for $\mathrm{O} 4$, we plan to only trigger on events with FARs lower than $1 \mathrm{yr}^{-1}$ (i.e., four out of 15 events in $\mathrm{O} 3$ would fail this criterion). We plan to only trigger on NSBH events with a nonzero HasRemnant probability (i.e., six out of eight NSBH triggers in $\mathrm{O} 3$ would fail this criterion, including GW190814). As we did in O3, we plan to only trigger on MassGap events with a nonzero HasNS probability. In summary, only five out of the 13 events followed up in $\mathrm{O} 3$ would pass our new plan for trigger criteria in O4.

The first phase of the ZTF survey ran from 2018 March to 2020 September. The second phase of the ZTF is expected to run from 2020 October to 2023 September. Searches with ZTF Phase II are planned to be up to 2 mag deeper than nominal survey operations, even with $1000 \mathrm{deg}^{2}$ localizations, thanks to the availability of deeper stacks as reference images. We plan to require a minimum median image depth of $-16.0 \mathrm{mag}$ and minimum enclosed probability of 50\% in the first $4 \mathrm{hr}$ of observations. The ZTF mapping speed allows $3600 \mathrm{deg}^{2}$ to be mapped in $4 \mathrm{hr}$ to achieve the necessary depth for a median GW distance of $300 \mathrm{Mpc}$. If the event is more distant, we will increase our exposure time from 180 to $600 \mathrm{~s}$ to go deeper. For events that are either too distant or too coarsely localized, we will not undertake triggered searches and will rely only on serendipitous searches of the all-sky public survey at 2 days' cadence to 20.4 mag.

Moreover, redder searches will better constrain the kilonova phase space and probe higher lanthanide fractions. The ZTF II would push to the red, since broader reference coverage is now available in the $i$-band filter (see Sagués Carracedo et al. 2020 for detailed simulations on gain in depth and red sensitivity). New wide-field infrared surveyors are also coming online (e.g., WINTER at Palomar Observatory, USA, and DREAMS at Siding Springs Observatory, Australia)

We look forward to searches in the fourth observing run, as detections will be more likely. For zero detections, about 17 neutron star mergers with only $50 \%$ enclosed probability to a depth of -16 mag would constrain the luminosity function fraction brighter than GW170817 to $<25 \%$ (only 11 events with $75 \%$ enclosed probability would place a similarly stringent limit). Thus, as the sample size grows, even with partial coverage of sky maps, the luminosity function of kilonovae will be strongly constrained.

We conclude with some thoughts on what would strengthen the partnership between the GW physics community and the EM astronomy community. First, we encourage efforts that would speed up the release of the more accurate LALInference map (Veitch et al. 2015). Since the LALInference map was often only available after our observations were completed, our net expectation value dropped by $10 \%$, and our net joint nondetection probability dropped by a factor of 2 between the BAYESTAR (Singer \& Price 2016) map and the LALInference map. Moreover, three triggers never had an LALInference map released (S190923y, S190930t, and S191205ah).
Second, it is critical that a reliable FAR and terrestrial probability are released as soon as possible. If an event is going to be retracted (or the FAR increases significantly) based on offline analysis, it is essential that the EM community be notified immediately via GCN, so that all pending follow-up can be halted. Third, if the classification of an event changes in offline analysis, the EM community should be promptly notified via GCN. Fourth, since HasNS and HasRemnant are somewhat model-dependent (e.g., Foucart et al. 2018; Chatterjee et al. 2020) but will drive the decision of whether or not some EM teams trigger follow-up, we request the release of rough estimates/ranges for more directly determined parameters (e.g., mass ratio, inclination, and chirp mass) that can help with the EM decision. We strongly encourage any algorithmic or technological development that will enable more accurate 3D skymaps, FARs, HasNS, and HasRemnant at lower latency to better inform the EM community's follow-up decisions.

In summary, the lessons learned from both the single detection in $\mathrm{O} 2$ and the dozen nondetections in $\mathrm{O} 3$ bode well for an exciting future for multimessenger astrophysics in the coming decade.

This work was supported by the Global Relay of Observatories Watching Transients Happen (GROWTH) project, funded by the National Science Foundation under PIRE grant No. 1545949. GROWTH is a collaborative project among the California Institute of Technology (USA), University of Maryland College Park (USA), University of Wisconsin Milwaukee (USA), Texas Tech University (USA), San Diego State University (USA), University of Washington (USA), Los Alamos National Laboratory (USA), Tokyo Institute of Technology (Japan), National Central University (Taiwan), Indian Institute of Astrophysics (India), Indian Institute of Technology Bombay (India), Weizmann Institute of Science (Israel), The Oskar Klein Centre at Stockholm University (Sweden), Humboldt University (Germany), Liverpool John Moores University (UK), and University of Sydney (Australia). Based on observations obtained with the Samuel Oschin Telescope 48 inch and the 60 inch telescope at the Palomar Observatory as part of the Zwicky Transient Facility project. The ZTF is supported by the National Science Foundation under grant No. AST-1440341 and a collaboration including Caltech, IPAC, the Weizmann Institute for Science, the Oskar Klein Center at Stockholm University, the University of Maryland, the University of Washington, Deutsches Elektronen-Synchrotron and Humboldt University, Los Alamos National Laboratories, the TANGO Consortium of Taiwan, the University of Wisconsin at Milwaukee, and Lawrence Berkeley National Laboratories. Operations are conducted by COO, IPAC, and UW. The ZTF forced photometry service was funded under Heising-Simons Foundation grant No. 12540303 (PI: Graham). The SED Machine is based upon work supported by the National Science Foundation under grant No. 1106171.

The GROWTH-India telescope is a $70 \mathrm{~cm}$ telescope with a $0.7^{\circ}$ field of view, set up by the Indian Institute of Astrophysics and the Indian Institute of Technology Bombay with support from the Indo-US Science and Technology Forum (IUSSTF) and the Science and Engineering Research Board (SERB) of the Department of Science and Technology (DST), Government of India (https://sites.google.com/view/growthindia/). 
It is located at the Indian Astronomical Observatory (Hanle), operated by the Indian Institute of Astrophysics (IIA). The GROWTH-India project is supported by SERB and administered by IUSSTF under grant No. IUSSTF/PIRE Program/ GROWTH/2015-16. This research has made use of the VizieR catalog access tool, CDS, Strasbourg, France (doi: 10.26093/ cds/vizier). The original description of the VizieR service was published in A\&AS 143, 23. These results made use of the Lowell Discovery Telescope (LDT) at Lowell Observatory. Lowell is a private, nonprofit institution dedicated to astrophysical research and public appreciation of astronomy and operates the LDT in partnership with Boston University, the University of Maryland, the University of Toledo, Northern Arizona University, and Yale University. The Large Monolithic Imager was built by Lowell Observatory using funds provided by the National Science Foundation (AST-1005313). The upgrade of the DeVeny optical spectrograph has been funded by a generous grant from John and Ginger Giovale and a grant from the Mt. Cuba Astronomical Foundation. The KPED team thanks the National Science Foundation and the National Optical Astronomical Observatory for making the Kitt Peak $2.1 \mathrm{~m}$ telescope available. We thank the observatory staff at Kitt Peak for their efforts to assist Robo-AO KP operations. The KPED team thanks the National Science Foundation, the National Optical Astronomical Observatory, the Caltech Space Innovation Council, and the Murty family for support in the building and operation of KPED. In addition, they thank the CHIMERA project for use of the Electron Multiplying CCD (EMCCD). The Liverpool Telescope is operated on the island of La Palma by Liverpool John Moores University in the Spanish Observatorio del Roque de los Muchachos of the Instituto de Astrofisica de Canarias with financial support from the UK Science and Technology Facilities Council. Some spectroscopic observations were obtained with the Southern African Large Telescope (SALT). The Photometric Redshifts for the Legacy Surveys (PRLS) catalog used in this paper was produced thanks to funding from the U.S. Department of Energy Office of Science, Office of High Energy Physics, via grant DE-SC0007914. This publication has made use of data collected at Lulin Observatory, partly supported by MoST grant 108-2112-M-008-001. Based on observations made with the Gran Telescopio Canarias (GTC), installed at the Spanish Observatorio del Roque de los Muchachos of the Instituto de Astrofísica de Canarias on the island of La Palma.

M.M.K. acknowledges generous support from the David and Lucille Packard Foundation. M.W.C. acknowledges support from the National Science Foundation with grant No. PHY-2010970. A.G. and J.S. acknowledge support from the Knut and Alice Wallenberg Foundation and GREAT research environment grant 2016-06012, funded by the Swedish Research Council. Some of the work by D.A.P. was performed at the Aspen Center for Physics, which is supported by National Science Foundation grant PHY-1607611. D.A.P. was partially supported by a grant from the Simons Foundation. H.K. thanks the LSSTC Data Science Fellowship Program, which is funded by LSSTC, NSF Cybertraining Grant 1829740, the Brinson Foundation, and the Moore Foundation; his participation in the program has benefited this work. This work has been supported by the Spanish Science Ministry Centro de Excelencia Severo Ochoa Program under grant SEV-2017-0709. A.J.C.T. acknowledges support from the Junta de Andalucía (Project P07-TIC-03094) and Spanish Ministry Projects AYA2012-39727-C03-01, AYA2015-71718R, and PID2019-109974RB-I00. V.A.F. was supported by grant RFBR 19-02-00432. I.A. acknowledges support by a Ramón y Cajal grant (RYC-2013-14511) of the Ministerio de Ciencia, Innovación, y Universidades (MICIU) of Spain. He also acknowledges financial support from MCIU through grant AYA2016-80889-P. A.A.M. is funded by the Large Synoptic Survey Telescope Corporation, the Brinson Foundation, and the Moore Foundation in support of the LSSTC Data Science Fellowship Program; he also receives support as a CIERA Fellow by the CIERA Postdoctoral Fellowship Program (Center for Interdisciplinary Exploration and Research in Astrophysics, Northwestern University). A.C. acknowledges support from the National Science Foundation with grant No. 1907975. W.-H.I., A. K., K.-L.L., C.-C.N., A.P., H.T., and P.-C.Y. acknowledge support from Ministry of Science and Technology (MoST) Taiwan grants 104-2923-M-008-004-MY5, 107-2119-M-008012, 108-2628-M-007-005-RSP, and 108-2112-M-007-025MY3. D.D. is supported by an Australian Government Research Training Program Scholarship. S.A. is supported by the GROWTH project, funded by the National Science Foundation under PIRE grant No. 1545949. A.S.C. is supported by GREAT research environment grant 2016-06012, funded by the Swedish Research Council. E.C.K. acknowledges support from the G.R.E. A.T. research environment and the Wenner-Gren Foundations. A. J.C.T. is thankful for fruitful discussions with J. Cepa, E. Fernández-García, J. A. Font, S. Jeong, A. Martín-Carrillo, A. M. Sintes, and S. Sokolov. D.A.H.B. acknowledges research support from the National Research Foundation of South Africa. S.B.P. and V.B. acknowledge BRICS grant No. "DST/IMRCD/ BRICS/PilotCall1/ProFCheap/2017(G)" for part of the present work. J.S.B. was partially supported by a Gordon and Betty Moore Foundation Data-Driven Discovery grant and a grant from the National Science Foundation, "Conceptualization of a Scalable Cyberinfrastructure Center for Multimessenger Astrophysics."

\section{Appendix A \\ Observing and Data Reduction Details for Follow-up Observations}

\section{A.1. Photometric Follow-up}

We used the 1 and $2 \mathrm{~m}$ telescopes available at the LCO global network to follow up sources discovered with the ZTF. The images were taken with the Sinistro and Spectral cameras (Brown et al. 2013) at the 1 and $2 \mathrm{~m}$, respectively, and scheduled through the LCO Observation Portal. ${ }^{76}$ The exposure time varied depending on the brightness of the object, yet our requests would normally involve three sets of $300 \mathrm{~s}$ in the $g$ and $r$ bands. After stacking the reduced images, we extracted sources using the SExtractor package (Bertin \& Arnouts 2010), and we calibrated magnitudes against PS1 (Chambers et al. 2016) objects in the vicinity. For nuclear transients located $<8^{\prime \prime}$ from their potential host, we use the High Order Transform of Psf ANd Template Subtraction code (HOTPANTS; Becker 2015) to subtract a PSF-scaled PS1 template previously aligned using SCAMP (Bertin 2006). The photometry for the nuclear candidates follows the same procedure described before but in the residual image. The images obtained with the LT were acquired using the IO:O camera with the Sloan griz filter set. They were reduced using the automated pipeline, which performs bias subtraction,

\footnotetext{
${ }^{76}$ https://observe.lco.global/
} 
trimming of the overscan regions, and flat-fielding. The image subtraction takes place once a PS1 template is aligned, and the final data come from the analysis of the subtracted image.

We used the Electronic Multiplier CCD camera at KPED to take hour-long exposures in the $r$ band to follow up candidates. After stacking the images and following standard reduction techniques, we calibrate the extracted sources using PS1 sources in the field. When the candidate has a host galaxy, we perform image subtraction as described for the LCO.

We obtained data with the GMOS-N (Allington-Smith et al. 2002; Hook et al. 2004; Gimeno et al. 2016), mounted on the Gemini-North $8 \mathrm{~m}$ telescope on Maunakea. Data were analyzed after stacking four $200 \mathrm{~s}$ exposures in the $g$ and $i$ bands. The reductions were performed using the python package DRAGONS $^{77}$ provided by the Gemini Observatory. We used PS1 sources in the field to calibrate the data.

We used the LOT at the Lulin Observatory in Taiwan to follow up candidates discovered with the ZTF. The standard observations involved $240 \mathrm{~s}$ in the $g^{\prime}, r^{\prime}$, and $i^{\prime}$ bands. The reduction followed standard methods, and the sources were calibrated against the PS1 catalog. No further image subtraction was applied to the images acquired with the LOT.

We used the $0.7 \mathrm{~m}$ robotic GIT equipped with a $4096 \times 4108$ pixel back-illuminated Andor camera for LVC event follow-up during O3. The GIT is situated at the IAO (Hanle, Ladakh). We used both tiled and targeted modes for the follow-up for different GW triggers. Tiled observations typically comprise a series of $600 \mathrm{~s}$ exposures in the Sloan Digital Sky Survey (SDSS) $r^{\prime}$ filter. Targeted observations were conducted with varying exposure times in the SDSS $u^{\prime}, g^{\prime}, r^{\prime}$, and $i^{\prime}$ filters. All data were downloaded in real time and processed with the automated GIT pipeline. Zero-points for photometry were calculated using the PanSTARRS catalog (Flewelling 2018), downloaded from Vizier. The PSF photometry was performed with PSFEx (Bertin 2011). For sources with significant host background, we performed image subtraction with pyzogy (Guevel \& Hosseinzadeh 2017), based on the ZOGY algorithm (Zackay et al. 2016).

Additionally, we obtained photometric data with the Spectral Energy Distribution Machine (SEDM; Blagorodnova et al. 2018; Rigault et al. 2019) on the P60 telescope. The processing is automated and can be triggered from the GROWTH Marshal. Standard requests involved $g_{-}, r$-, and $i$-band imaging with the Rainbow Camera on the SEDM in $300 \mathrm{~s}$ exposures. The data are later reduced using a python-based pipeline that applies standard reduction techniques and a customized version of the Fremling Automated Pipeline (FPipe; Fremling et al. 2016) for image subtraction.

We used the imaging capabilities of the OSIRIS (Cepa et al. 2005) camera at the GTC to obtain $60 \mathrm{~s}$ exposures in the $r$ band. Standard reduction techniques were applied to the data, and we used PS1 sources to calibrate the flux.

We obtained follow-up imaging of candidates with the Wafer Scale Imager for Prime (WASP) and the Wide-field Infrared Camera (WIRC; Wilson et al. 2003), both on the P200 telescope. For WASP data, a python-based pipeline applied standard optical reduction techniques (as described in De et al. 2020a), and the photometric calibration was obtained against PS1 sources in the field. The WIRC data were treated similarly using the same pipeline, but they were additionally stacked

\footnotetext{
77 https://dragons.readthedocs.io/en/stable/
}

using Swarp (Bertin et al. 2002), while the calibration was done using the Two Micron All Sky Survey point-source catalog (Skrutskie et al. 2006).

We obtained imaging of one candidate using the Low Resolution Imaging Spectrometer (LRIS; Oke et al. 1995), mounted at the Keck I telescope. Our data were taken in the $g$ and $i$ bands reaching $m_{\mathrm{AB}} \approx 24$. The data were reduced following standard methods.

We used the Large Monolithic Imager (LMI; Massey et al. 2013) on the $4.3 \mathrm{~m}$ LDT at Happy Jack, Arizona, to follow up the ZTF discoveries. Observations were conducted with the SDSS $r$ filter for $90 \mathrm{~s}$ each, and the data were reduced using the photopipe ${ }^{78}$ pipeline. The magnitudes were calibrated against the SDSS or Gaia catalogs (Ahumada et al. 2020) using the conversion scheme provided in Gaia documentation. ${ }^{79}$

We used the Ultraviolet/Optical Telescope (UVOT; Roming et al. 2005) mounted on the Neil Gehrels Swift Observatory (hereafter referred to as Swift; Gehrels et al. 2004) to follow up interesting sources and track down their UV evolution. The ToO observations were scheduled in the $v, b, u, w 1, m 2$, and $w 2$ bands for an average of $320 \mathrm{~s}$ exposure ${ }^{-1}$. We used the products of the Swift pipeline to determine the magnitudes. ${ }^{80}$

We observed candidate counterparts of S200213t using the Astrophysical Research Consortium Telescope Imaging Camera (ARCTIC; Huehnerhoff et al. 2016) at the APO $3.5 \mathrm{~m}$. We obtained dithered $120 \mathrm{~s}$ exposures binned $2 \times 2$ in the $u, g, r, i$, and $z$ bands. Images were bias-corrected, flat-fielded, and combined using standard IRAF packages (noao, imred, and ccdred). SExtractor (Bertin \& Arnouts 2010) was used to find and photometer point sources in the images using PSF photometry, and a photometric calibration to PanSTARRS field stars was performed (without filter corrections).

All photometry presented in the light curves and tables in this paper is corrected for galactic extinction using dust maps from Schlafly \& Finkbeiner (2011).

We observed the field of ZTF19aassfws with the VLA in its B configuration on 2019 May 10 starting at 07:19:15 UT and 2019 June 4 starting at 08:20:32 UT. Our observations were carried out at a nominal central frequency of $3 \mathrm{GHz}$. We used 3C 286 as our bandpass and absolute flux calibrator and J1927 +6117 as our complex gain calibrator. Data were calibrated using the standard VLA automated calibration pipeline available in the Common Astronomy Software Applications (CASA) package. We then inspected the data for further flagging and imaged interactively using the CLEAN algorithm. The image rms was $\approx 5.2 \mu \mathrm{Jy}$ for the first epoch and $\approx 4.6 \mu \mathrm{Jy}$ for the second epoch. Within a circular region centered on the optical position of ZTF19aassfws and of radius $\approx 2$ !! 1 (comparable to the nominal half-power beamwidth of the VLA at $3 \mathrm{GHz}$ and for the $\mathrm{B}$ configuration), we find no significant radio emission. Thus, we set upper limits on the corresponding $3 \mathrm{GHz}$ flux density of $\lesssim 16$ and $\lesssim 14 \mu \mathrm{Jy}$, respectively, for the first and second epochs.

\section{A.2. Spectroscopic Follow-up}

Using the GROWTH Marshal, we regularly triggered the Liverpool Telescope Spectrograph for the Rapid Acquisition of

\footnotetext{
78 https://github.com/maxperry/photometrypipeline

79 https://gea.esac.esa.int/archive/documentation/GDR2/Data_processing/ chap_cu5pho/sec_cu5pho_calibr/ssec_cu5pho_PhotTransf.html

80 https://swift.gsfc.nasa.gov/quicklook/
} 
Transients (SPRAT; Piascik et al. 2014). SPRAT uses a 1".8 slit, which provides a resolution of $R=350$ at the center of the spectrum. The data were reduced using the automated pipeline, which removes low-level instrumental signatures and then performs source extraction, sky subtraction, and wavelength and flux calibration.

We observed a number of transient candidates during classical observing runs with the P200 Double Spectrograph (DBSP) during O3. For the setup configuration, we used 1". 0 , 1 ". 5 , and $2^{\prime \prime}$ slit masks; a D55 dichroic; a blue grating of $600 /$ 4000; and a red grating of 316/7500. Using a custom PyRAF DBSP reduction pipeline (Bellm \& Sesar 2016), ${ }^{81}$ we reduced our data.

We obtained several optical spectra with the $10.4 \mathrm{~m} \mathrm{GTC}$ telescope (equipped with OSIRIS). We used the R1000B and R500R grisms for our observations, typically using a slit of width of 1 !!2. We used standard routines from the Image Reduction and Analysis Facility (IRAF) to perform our data reduction.

We observed ZTF19aarykkb using the DeVeny spectrograph mounted on the $4.3 \mathrm{~m}$ LDT. We obtained 22.5 minute exposures at an average airmass of 1.5 . We used the DV2 grating $\left(300 \mathrm{~g} \mathrm{~mm}^{-1}\right.$, $4000 \AA$ blaze) for this observation. Our spectra cover a wavelength range of approximately 3600-8000 .

In addition, we obtained a spectrum of ZTF20aarzaod with SALT (Buckley et al. 2003), using the Robert Stobie Spectrograph (RSS; Burgh et al. 2003), covering a wavelength range of 470-760 nm with a spectral resolution of $R=400$. We triggered special GW follow-up program 2018-2-GWE002 and reduced the data with a custom pipeline based on PyRAF routines and the PySALT package (Crawford et al. 2010).

Low-resolution spectra using the $2 \mathrm{~m}$ HCT were obtained using the HFOSC instrument; ZTF19aarykkb was observed using grisms Gr7 (3500-7800 $)$ and Gr8 (5200-9000 ̊), while AT2019wxt was observed using Gr7. The spectra were bias-subtracted, cosmic rays were removed, and the 1D spectra were extracted using the optimal extraction method. Wavelength calibration was effected using the arc lamp spectra $\mathrm{FeAr}$ (Gr7) and FeNe (Gr8). Instrumental response curves generated using spectrophotometric standards observed during the same night were used to calibrate the spectra onto a relative flux scale. The flux-calibrated spectra of ZTF19aarykkb from the two grisms were combined to a single spectrum covering the wavelength range 4000-9000 A.

We obtained spectroscopy with the GMOS-N, mounted on the Gemini-North $8 \mathrm{~m}$ telescope on Maunakea, by combining six 450 s exposures on the R400 and B600 gratings. We used the GMOS long-slit capability and reduced the data following standard PyRAF techniques.

We obtained near-infrared spectroscopy of candidates using NIRES on the Keck II telescope. The data were acquired using standard ABBA dither patterns on the target source, followed by observations of an A0 telluric standard star close to the science target. The spectral traces were extracted using the spextool package (Cushing et al. 2004) for both the science target and standard star. The final spectra presented here were stacked from all individual dithers, followed by flux calibration and telluric correction using the xtellcor package (Vacca et al. 2003).

\footnotetext{
${ }^{81}$ https://github.com/ebellm/pyraf-dbsp
}

We obtained spectra using the LRIS on the Keck I telescope. The 600/4000 grism was used on the blue side and the 600/ 7500 grating on the red side, providing wavelength coverage between 3139-5642 $\AA$ (blue) and 6236-9516 $\AA$ (red). The exposure time was $600 \mathrm{~s}$ on both sides. The spectrum was reduced using LPipe (Perley 2019) with BD+28 as a flux calibrator. The red and blue relative fluxes are scaled by matching synthetic photometry to colors inferred from photometry of the transient.

\section{Appendix B Detailed Candidate Descriptions}

Here we provide descriptions of each candidate identified within the sky map of each event followed up with the ZTF. We discuss each object announced via GCN. For candidates with a redshift, we note whether it is spectroscopic $[s]$ or photometric $[p]$. Some candidates were classified as a part of coordinated spectroscopic follow-up with the Bright Transient Survey (BTS; Fremling et al. 2020) and the ZTF CLU experiment (De et al. 2020b).

\section{B.1. GW190425}

For candidates identified within the sky map of GW190425, see Coughlin et al. (2019a). Two candidate counterparts of GW190425z, ZTF19aarykkb and ZTF19aarzoad, were observed with the AMI Large Array at $15 \mathrm{GHz}$ on 2019 April 26 (Rhodes et al. 2019). No radio emission was found to be associated with any of these candidates.

\section{B.2. $S 190426 c$}

We summarize the candidate counterparts to S190426c in Table 2 and the follow-up photometry in Table 11. Next, we discuss why we conclude that each one is unrelated.

\section{B.2.1. Spectroscopically Classified}

ZTF19aasmftm/AT2019sne-The rising light curve of ZTF19aasmftm suggested that it could be a young and faint object with a galaxy host of $m_{\mathrm{AB}}=21.2 \mathrm{mag}$ in PS1, so we highlighted it in Perley et al. (2019a). A few days later, GTC spectroscopy of this event (Hu et al. 2019b) classified it as a premaximum SN Ia in the outskirts of its host galaxy at $z$ $[s]=0.156$.

ZTF19aaslzif/AT2019snh-Another candidate discovered during our second night of observations, ZTF19aaslzjf, was at low galactic latitude and seemed to be located in a nearby host galaxy. A spectrum from GTC (Hu et al. 2019b) confirmed both that this source was nearby (at $z[s]=0.086$ ) and that it was an SN Ia located in the outskirts of the Galaxy host.

ZTF19aasmddt/SN2019fht-We highlighted this transient because its photometric redshift was consistent with the LVC distance estimate, and the light curve exhibited a rapid rise (Perley et al. 2019a). However, the GTC spectrum taken shortly afterward revealed that this transient was a young SN II prepeak in the outskirts of its galaxy at $z[s]=0.028$.

ZTF19aaslszp/AT2019anj-Another candidate whose photo-z was consistent with the LVC distance estimate, ZTF19aaslszp, appeared to be relatively bright and red with a color of $g-r=0.89$ mag. Subsequent ZTF and LT photometry revealed that the source appeared to have flaring behavior in the light 
Table 11

Follow-up Photometry for S190426c Candidates

\begin{tabular}{|c|c|c|c|c|c|c|c|}
\hline Name & IAU Name & Date & Telescope & Filter & $m(\mathrm{AB})$ & $\sigma_{m}$ & $m_{\lim }$ \\
\hline ZTF19aasmftm & AT2019sne & $2,458,602.6514$ & LT & $g$ & 21.33 & 0.15 & 21.71 \\
\hline ZTF19aasmftm & AT2019sne & $2,458,602.6528$ & LT & $r$ & 21.06 & 0.10 & 21.51 \\
\hline ZTF19aasmftm & AT2019sne & $2,458,602.6542$ & LT & $i$ & 20.90 & 0.17 & 21.03 \\
\hline ZTF19aassfws & AT2019fuc & $2,458,603.6605$ & LT & $g$ & 99.0 & 99.0 & 22.32 \\
\hline ZTF19aassfws & AT2019fuc & $2,458,603.6619$ & LT & $r$ & 99.0 & 99.0 & 22.04 \\
\hline ZTF19aassfws & AT2019fuc & $2,458,603.6633$ & LT & $i$ & 99.0 & 99.0 & 21.50 \\
\hline ZTF19aaslszp & AT2019snj & $2,458,603.6654$ & LT & $g$ & 20.80 & 0.07 & 22.25 \\
\hline ZTF19aaslszp & AT2019snj & $2,458,603.6668$ & LT & $r$ & 20.51 & 0.07 & 22.12 \\
\hline ZTF19aaslszp & AT2019snj & $2,458,603.6682$ & LT & $i$ & 19.19 & 0.06 & 22.00 \\
\hline ZTF19aaslzjf & AT2019snh & $2,458,603.6703$ & LT & $g$ & 20.94 & 0.18 & 21.75 \\
\hline ZTF19aaslzjf & AT2019snh & $2,458,603.6717$ & LT & $r$ & 20.40 & 0.10 & 22.00 \\
\hline ZTF19aaslzjf & AT2019snh & $2,458,603.6731$ & $\mathrm{LT}$ & $i$ & 20.30 & 0.10 & 22.00 \\
\hline ZTF19aasmddt & SN2019fht & $2,458,603.7113$ & $\mathrm{LT}$ & $g$ & 19.79 & 0.10 & 22.77 \\
\hline ZTF19aasmddt & SN2019fht & $2,458,603.7127$ & LT & $r$ & 19.43 & 0.11 & 21.54 \\
\hline ZTF19aasmddt & SN2019fht & $2,458,603.7141$ & LT & $i$ & 19.41 & 0.09 & 21.10 \\
\hline ZTF19aasmddt & SN2019fht & $2,458,604.7237$ & $\mathrm{LT}$ & $g$ & 19.69 & 0.06 & 21.61 \\
\hline ZTF19aasmddt & SN2019fht & $2,458,604.7251$ & LT & $r$ & 19.51 & 0.03 & 22.29 \\
\hline ZTF19aasmddt & SN2019fht & $2,458,604.7265$ & LT & $i$ & 19.55 & 0.07 & 20.63 \\
\hline
\end{tabular}

curve. Our P200+DBSP spectrum classified the source as an AGN at $z[s]=0.084$, as it shows broad hydrogen lines.

\section{B.2.2. Slow Photometric Evolution}

ZTF19aaslzfk/AT2019snd-We identified this candidate during our initial search of the imaged region within the BAYESTAR localization of S190426c (Coughlin et al. 2019c). Though the candidate had WISE detections in all four filters, its WISE colors did not definitively place this transient in the AGN class. Continued photometric monitoring of this candidate revealed its slow evolution $\left(\alpha_{g}=-0.02\right)$, ruling out its association with S190426c.

ZTF19aaslvwn/AT2019snf - We reported ZTF19aaslvwn in Perley et al. (2019a) as a lower-priority transient, with initially slow photometric evolution at low galactic latitude $\left(b<15^{\circ}\right)$. After monitoring the transient over a period of $\sim 12$ days, the photometry had only risen by $0.4 \mathrm{mag}$, indicating that it could not be a kilonova and was likely a CV.

ZTF19aasmdir/AT2019sng-Also reported in Perley et al. (2019a), ZTF19aasmdir was a nuclear transient at a low galactic latitude, with WISE colors consistent with an AGN within $1^{\prime \prime}$ of the transient. Several days of monitoring yielded a light curve that was far more consistent with a flaring AGN than a kilonova, with a rate of evolution $\alpha_{r}<0.01$.

ZTF19aaslolf/AT2019snn-This nuclear candidate was a low priority in our follow-up list due to its high photometric redshift $(z[p]=0.42)$ and because its WISE colors placed it within the AGN locus. Though we could not spectroscopically confirm this, the slowly evolving "flaring" light curve $\left(\alpha_{r}<0.01\right)$ and archival PS1 detections point to the AGN nature of this candidate.

ZTF19aaslphi/AT2019sno-The candidate ZTF19aaslphi had a photometric redshift that was also nominally inconsistent with the LVC distance. However, we identified it as a candidate of interest due to its relatively quick rise of $\sim 0.75 \mathrm{mag}$ over the course of 4 days in the $g$ band. Its later-time light curve exhibited a plateau; thus, we consider its evolution too slow to be associated with a $\mathrm{GW}$ event.
ZTF19aaslpds/AT2019snq-This candidate, at low galactic latitudes, had multiple detections in the $r$ and $g$ filters; but, as it only evolved by $0.04 \mathrm{mag}$ over a day of monitoring and subsequently was not detected, we ruled it out as a potential counterpart to S190426c.

ZTF19aaslozu/AT2019snr-We included this candidate initially due to its rapid rise and $g-r$ color of 0.3 mag (Perley et al. 2019a). Though ZTF19aaslozu did not clearly fall into the AGN locus, its detections in all four WISE filters, archival detections with PS1, and slow evolution point to it being a strong AGN candidate.

ZTF19aasshpf/AT2019snt-This is a lower-priority candidate on our list discovered at $r=21.59$ mag in the outskirts of a faint red galaxy. It exhibited a flat evolution (0.06 mag) over a period of 27 days, thus ruling out its association with S190426c.

ZTF19aasmzqf - We could likewise rule out the possibility of ZTF19aasmzqf being a kilonova due to its slow evolution of 0.3 mag over 28 days, despite its initial red color $g-r=0.22$ mag.

\section{B.2.3. Stellar}

ZTF19aasmekb/AT2019snl_-Located at low galactic latitude ( $b=-8.64)$, ZTF19aasmekb appeared to be hostless and initially exhibited a rapid fade; its later-time light curve is photometrically consistent with a $\mathrm{CV}$, and its slow evolution $\left(\alpha_{g}=0.24\right)$ is inconsistent with a kilonova origin.

\section{B.2.4. Artifacts}

ZTF19aassfws/AT2019fuc-We highlighted ZTF19aassfws as a candidate of potential interest because its photometric redshift fell within the LIGO distance uncertainty (Perley et al. 2019a). We also obtained radio follow-up using the VLA and AMI under the Jansky VLA mapping of Gravitational Waves as Afterglows in Radio (JAGWAR; Mooley et al. 2018), and we did not detect any radio emission. However, upon careful inspection of the reference image, we identified a very subtle gain mismatch across the image. Comparing the initial 
photometry of the transient with the level of the gain mismatch provided a clear indication that our candidate was not astrophysical but rather an artifact. This gain mismatch problem has since been fixed by rebuilding the references.

\section{B.3. GW190814}

No candidates were identified in the ZTF follow-up of the small localization of GW190814.

\section{B.4. S190901ap}

We summarize the candidate counterparts to S190901ap in Table 3 and the follow-up photometry in Table 12. Next, we discuss why we conclude that each one is unrelated.

\section{B.4.1. Spectroscopically Classified}

ZTF19abvizsw/AT2019pim-We discovered a red transient ( $g-r \approx 0.5$ ) that appeared to be hostless and fast-evolving. We had observed the location of this transient every night for the month leading up to 2019 September 1 with no previous detections, therefore indicating strongly that this object was a new transient. Gravitational Wave Inaf Team Collaboration (GRAWITA) spectroscopic observations about $10 \mathrm{hr}$ later seemed to suggest that the object was a galactic K or M dwarf (Salsamo et al. 2019a), but our subsequent LRIS spectroscopic follow-up yielded a featureless continuum with $\mathrm{Mg}$ II, $\mathrm{Mg}$ I, and Fe II lines at $z[s]=1.26$ (Burdge et al. 2019). Thus, we posited that the object could be a flaring AGN or a GRB afterglow. Observations with SVOM-GWAC-F60A (Wei et al. 2019) and the LT (Perley et al. 2019b) indicated that the light curve was rapidly decaying, suggesting that the transient was likely an orphan GRB afterglow. More than 10 other GCNs contained reported follow-ups of this transient; the collated evidence posed the coherent picture that we had, remarkably, detected an untriggered long GRB afterglow in temporal and spatial coincidence with the sky map of S190901ap. This candidate will be discussed in more detail in D. A. Perley et al. (2020, in preparation).

ZTF19abvixoy/AT2019pin-We detected this transient with an upper limit from the day before the merger, though it appeared to have a faint counterpart in PS1. GRAWITA spectroscopic observations classified this transient as a CV due to its blue continuum and weak $\mathrm{H} \alpha$ emission surrounded by broad absorption troughs (Salmaso et al. 2019b).

ZTF19abvionh/AT2019pip-The photometric redshift of the putative host of this transient initially made it an interesting candidate for association with S190901ap, even though its first two detections were separated by a short baseline of 7 minutes. About $15 \mathrm{hr}$ later, spectroscopic observations with the HobbyEberly observatory suggested that the host galaxy GALEXASC $\mathrm{J} 165500.03+140301.3$ was located at a distance of $\sim 450 \mathrm{Mpc}$ (Rosell et al. 2019); our LRIS spectrum, showing a hot blue continuum and host galaxy lines at $z[s]=0.0985$, confirmed this conclusion, placing the transient outside of the $\mathrm{GW}$ distance error bar by $2.5 \sigma$. Upon close inspection of the spectra, we find $\mathrm{H} \alpha$ and $\mathrm{He}$ II at zero redshift, suggesting that the transient is a foreground $\mathrm{CV}$ and the background host galaxy is unrelated.

ZTF19abwvals/AT2019pni-Another transient detected via the AMPEL alert archive, ZTF19abwvals, appeared to be red $(g-r \sim 0.5)$ and had a photometric redshift of 0.13 , slightly higher than the GW distance, also with upper limits in the $g$ band the previous day (Stein et al. 2019c). The SNID template matching to the spectra taken with the ALFOSC spectrograph on the Nordic Optical Telescope revealed that ZTF19abwvals was a normal SN Ia, about 4-6 days postpeak (Izzo et al. 2019).

\section{B.4.2. Slow Photometric Evolution}

ZTF19abwsmmd/AT2019pnc-Further searches of the data with the AMPEL pipeline yielded two additional candidates, including ZTF19abwsmmd (Stein et al. 2019c). This candidate exhibited a blue color $(g-r \sim 0.25)$ and had nondetections in the $g$ band to 20.64 mag a day before the merger. The ZTF survey operations monitored it over a period of about 35 days. The light curve exhibited a change of only 0.2 mag decline over that baseline; therefore, we deemed it too slow to be associated with the GW event.

ZTF19abvislp/AT2019pnx-We performed a second search of the AMPEL alert archive in which we identified this transient, detected on the first night of observations. It was interesting due to its rising light curve and host SDSS galaxy being at a redshift of 0.1 , on the upper end of the LIGO distance range. Instead of using our spectroscopic resources, we chose to monitor the transient photometrically, and its evolution over nearly 30 days proved to be too slow $\left(\alpha_{r}=0.05\right)$ to be a kilonova.

ZTF19abxdvcs/AT2019qev-We also discovered ZTF19abxdvcs during a second AMPEL archive search and highlighted it due to its photometric redshift $(z \sim 0.118)$ and the fact that it had risen by more than 0.65 mag over the course of 3 days, with its first detection on the first night. Though we did not report this candidate via GCN, our continued photometric monitoring with the ZTF demonstrated that the transient was evolving with $\alpha_{g}=0.03$, and its light curve resembled that of an $\mathrm{SN}$, so we could confidently reject it.

\section{B.5. S190910d}

We summarize the candidate counterparts to S190910d in Table 4 and the follow-up photometry in Table 13. Next, we discuss why we conclude that each one is unrelated.

\section{B.5.1. Spectroscopically Classified}

ZTF19abyfhov/AT2019pvu-We identified this candidate during our follow-up campaign for S190910d with no available photometric redshifts due to cross-matches at its sky position (Anand et al. 2019). Castro-Tirado et al. (2019b) observed it with the $10.4 \mathrm{~m}$ GTC telescope equipped with OSIRIS in La Palma, Spain, about $16 \mathrm{hr}$ after initial detection and derived an $r$-band magnitude of $20.33 \mathrm{mag}$ for the transient. The best match to their spectrum indicated that the candidate was an SN Ia at $z[s]=0.133 \pm 0.001$. Another spectrum taken with the ACAM instrument on the William Herschel Telesope at the Roque de los Muchachos Observatory in La Palma confirmed the classification (Cannizzaro et al. 2019).

ZTF19abyfhaq/AT2019pvv-Similarly, we detected ZTF19abyfhaq with little other information than the $r$-band magnitude of its initial detection at $20.3 \mathrm{mag}$ (Anand et al. 2019). The GTC spectrum taken (Castro-Tirado et al. 2019b) about $18 \mathrm{hr}$ after the initial detection had a too-low $\mathrm{S} / \mathrm{N}$ to merit a classification, but an $\mathrm{H} \alpha$ emission line at $z[s]=0$ revealed that the transient was galactic and therefore unrelated. 
Table 12

Follow-up Photometry for S190901ap Candidates

\begin{tabular}{|c|c|c|c|c|c|c|c|}
\hline Name & IAU Name & Date & Telescope & Filter & $m(\mathrm{AB})$ & $\sigma_{m}$ & $m_{\mathrm{lim}}$ \\
\hline ZTF19abvizsw & AT2019pim & $2,458,729.229$ & GIT & $i$ & 20.14 & 0.1 & 20.41 \\
\hline ZTF19abvizsw & AT2019pim & $2,458,729.126$ & GIT & $i$ & 20.13 & 0.09 & 20.41 \\
\hline ZTF19abvizsw & AT2019pim & $2,458,729.303$ & GIT & $g$ & 21.19 & 0.06 & 21.43 \\
\hline ZTF19abvizsw & AT2019pim & $2,458,729.103$ & GIT & $r$ & 20.57 & 0.11 & 20.65 \\
\hline ZTF19abvizsw & AT2019pim & $2,458,730.4481$ & LT & $g$ & 22.02 & 0.10 & 22.00 \\
\hline ZTF19abvizsw & AT2019pim & $2,458,730.4420$ & LT & $r$ & 21.62 & 0.09 & 22.0 \\
\hline ZTF19abvizsw & AT2019pim & $2,458,730.4541$ & $\mathrm{LT}$ & $i$ & 21.16 & 0.07 & 22.00 \\
\hline ZTF19abvizsw & AT2019pim & $2,458,730.4621$ & $\mathrm{LT}$ & $z$ & 20.87 & 0.12 & 22.00 \\
\hline ZTF19abvizsw & AT2019pim & $2,458,731.14$ & GIT & $i$ & 99.0 & 99.0 & 20.29 \\
\hline ZTF19abvizsw & AT2019pim & $2,458,731.134$ & GIT & $i$ & 99.0 & 99.0 & 20.29 \\
\hline ZTF19abvizsw & AT2019pim & $2,458,731.118$ & GIT & $r$ & 99.0 & 99.0 & 20.98 \\
\hline ZTF19abvizsw & AT2019pim & $2,458,731.125$ & GIT & $r$ & 99.0 & 99.0 & 21.14 \\
\hline ZTF19abvizsw & AT2019pim & $2,458,731.3862$ & $\mathrm{LT}$ & $g$ & 22.50 & 0.20 & 22.50 \\
\hline ZTF19abvizsw & AT2019pim & $2,458,731.3802$ & LT & $r$ & 22.05 & 0.10 & 22.50 \\
\hline ZTF19abvizsw & AT2019pim & $2,458,731.3923$ & LT & $i$ & 21.60 & 0.10 & 22.50 \\
\hline ZTF19abvizsw & AT2019pim & $2,458,731.3983$ & $\mathrm{LT}$ & $z$ & 21.20 & 0.20 & 22.50 \\
\hline ZTF19abvizsw & AT2019pim & $2,458,731.5172$ & $\mathrm{LT}$ & $g$ & 22.54 & 0.16 & 23.00 \\
\hline ZTF19abvizsw & AT2019pim & $2,458,731.5112$ & $\mathrm{LT}$ & $r$ & 22.10 & 0.12 & 23.00 \\
\hline ZTF19abvizsw & AT2019pim & $2,458,731.5232$ & $\mathrm{LT}$ & $i$ & 21.64 & 0.11 & 23.00 \\
\hline ZTF19abvizsw & AT2019pim & $2,458,731.5293$ & $\mathrm{LT}$ & $z$ & 21.55 & 0.22 & 23.00 \\
\hline ZTF19abvizsw & AT2019pim & $2,458,732.102$ & GIT & $r$ & 99.0 & 99.0 & 19.32 \\
\hline ZTF19abvizsw & AT2019pim & $2,458,732.119$ & GIT & $i$ & 99.0 & 99.0 & 20.4 \\
\hline ZTF19abvizsw & AT2019pim & $2,458,732.125$ & GIT & $i$ & 99.0 & 99.0 & 20.43 \\
\hline ZTF19abvizsw & AT2019pim & $2,458,738.3819$ & WHT & $r$ & 22.60 & 0.12 & 24.00 \\
\hline ZTF19abvizsw & AT2019pim & $2,458,739.3839$ & WHT & $i$ & 22.43 & 0.12 & 24.10 \\
\hline ZTF19abvizsw & AT2019pim & $2,458,740.4939$ & WHT & $i$ & 22.51 & 0.15 & 23.50 \\
\hline ZTF19abvizsw & AT2019pim & $2,458,740.5219$ & WHT & $r$ & 23.38 & 0.25 & 23.70 \\
\hline ZTF19abvizsw & AT2019pim & $2,458,750.7337$ & Keck1 & $g$ & 23.99 & 0.10 & 26.00 \\
\hline ZTF19abvizsw & AT2019pim & $2,458,750.7342$ & Keck1 & $i$ & 23.80 & 0.09 & 25.00 \\
\hline ZTF19abvionh & AT2019pip & $2,458,729.166$ & GIT & $r$ & 20.8 & 0.05 & 21.27 \\
\hline ZTF19abvionh & AT2019pip & $2,458,729.206$ & GIT & $r$ & 20.77 & 0.06 & 21.17 \\
\hline ZTF19abvionh & AT2019pip & $2,458,729.213$ & GIT & $r$ & 20.68 & 0.08 & 21.15 \\
\hline ZTF19abvionh & AT2019pip & $2,458,730.166$ & GIT & $r$ & 20.63 & 0.04 & 21.22 \\
\hline ZTF19abvionh & AT2019pip & $2,458,730.18$ & GIT & $r$ & 20.66 & 0.05 & 21.23 \\
\hline ZTF19abvionh & AT2019pip & $2,458,731.204$ & GIT & $g$ & 20.56 & 0.06 & 21.16 \\
\hline ZTF19abvionh & AT2019pip & $2,458,731.4331$ & $\mathrm{LT}$ & $u$ & 20.47 & 0.11 & 21.86 \\
\hline ZTF19abvionh & AT2019pip & $2,458,731.4208$ & $\mathrm{LT}$ & $g$ & 20.51 & 0.29 & 22.55 \\
\hline ZTF19abvionh & AT2019pip & $2,458,731.4168$ & $\mathrm{LT}$ & $r$ & 20.36 & 0.09 & 22.35 \\
\hline ZTF19abwsmmd & AT2019pnc & $2,458,731.5587$ & $\mathrm{LT}$ & $g$ & 19.86 & 0.16 & 20.41 \\
\hline ZTF19abwsmmd & AT2019pnc & $2,458,731.5641$ & $\mathrm{LT}$ & $r$ & 20.02 & 0.07 & 22.02 \\
\hline ZTF19abwsmmd & AT2019pnc & $2,458,731.5614$ & $\mathrm{LT}$ & $i$ & 20.26 & 0.06 & 22.47 \\
\hline ZTF19abwvals & AT2019pni & $2,458,731.7095$ & $\mathrm{LT}$ & $g$ & 20.42 & 0.07 & 22.63 \\
\hline ZTF19abwvals & AT2019pni & $2,458,731.7149$ & $\mathrm{LT}$ & $r$ & 20.04 & 0.08 & 22.96 \\
\hline ZTF19abwvals & AT2019pni & $2,458,731.7122$ & $\mathrm{LT}$ & $i$ & 20.23 & 0.24 & 22.30 \\
\hline ZTF19abvixoy & AT2019pin & $2,458,729.144$ & GIT & $r$ & 18.97 & 0.03 & 21.16 \\
\hline ZTF19abvixoy & AT2019pin & $2,458,729.182$ & GIT & $r$ & 18.73 & 0.02 & 21.17 \\
\hline ZTF19abvixoy & AT2019pin & $2,458,729.238$ & GIT & $i$ & 18.97 & 0.05 & 20.35 \\
\hline ZTF19abvixoy & AT2019pin & $2,458,729.245$ & GIT & $i$ & 19.06 & 0.05 & 20.38 \\
\hline ZTF19abvixoy & AT2019pin & $2,458,729.285$ & GIT & $i$ & 19.02 & 0.1 & 20.27 \\
\hline ZTF19abvixoy & AT2019pin & $2,458,729.292$ & GIT & $i$ & 18.94 & 0.1 & 20.23 \\
\hline ZTF19abvislp & AT2019pnx & $2,458,734.171$ & GIT & $g$ & 99.0 & 99.0 & 20.42 \\
\hline ZTF19abvislp & AT2019pnx & $2,458,734.178$ & GIT & $g$ & 99.0 & 99.0 & 20.29 \\
\hline ZTF19abvislp & AT2019pnx & $2,458,735.113$ & GIT & $g$ & 99.0 & 99.0 & 20.34 \\
\hline ZTF19abvislp & AT2019pnx & $2,458,735.181$ & GIT & $r$ & 99.0 & 99.0 & 19.91 \\
\hline ZTF19abvislp & AT2019pnx & $2,458,733.111$ & GIT & $g$ & 99.0 & 99.0 & 20.45 \\
\hline ZTF19abvislp & AT2019pnx & $2,458,733.118$ & GIT & $g$ & 99.0 & 99.0 & 20.36 \\
\hline ZTF19abvislp & AT2019pnx & $2,458,735.174$ & GIT & $r$ & 99.0 & 99.0 & 19.89 \\
\hline ZTF19abxdvcs & AT2019qev & $2,458,733.133$ & GIT & $g$ & 19.93 & 0.03 & 20.7 \\
\hline ZTF19abxdvcs & AT2019qev & $2,458,733.173$ & GIT & $r$ & 20.18 & 0.05 & 20.72 \\
\hline ZTF19abxdvcs & AT2019qev & $2,458,733.179$ & GIT & $r$ & 20.28 & 0.03 & 20.82 \\
\hline
\end{tabular}


Table 12

(Continued)

\begin{tabular}{llccccc}
\hline \hline Name & IAU Name & Date & Telescope & Filter & $m($ AB) & $\sigma_{m}$ \\
\hline ZTF19abxdvcs & AT2019qev & $2,458,734.242$ & GIT & $g$ & 19.83 & 0.03 \\
ZTF19abxdvcs & AT2019qev & $2,458,734.249$ & GIT & $g$ & 19.9 & 0.05 \\
ZTF19abxdvcs & AT2019qev & $2,458,734.258$ & GIT & $r$ & 20.03 & 0.05 \\
ZTF19abxdvcs & AT2019qev & $2,458,734.264$ & GIT & $r$ & 20.11 & 0.38 \\
ZTF19abxdvcs & AT2019qev & $2,458,735.206$ & GIT & $r$ & 19.8 & 0.05 \\
ZTF19abxdvcs & AT2019qev & $2,458,735.213$ & GIT & $r$ & 19.84 & 0.05 \\
\hline
\end{tabular}

Table 13

Follow-up Photometry for S190910d Candidates

\begin{tabular}{|c|c|c|c|c|c|c|c|}
\hline Name & IAU Name & Date & Telescope & Filter & $m(\mathrm{AB})$ & $\sigma_{m}$ & $m_{\lim }$ \\
\hline$\overline{\text { ZTF19abyfazm }}$ & AT2019pwa & $2,458,736.8848$ & P60 & $r$ & 18.17 & 0.04 & 20.48 \\
\hline ZTF19abyfazm & AT2019pwa & $2,458,737.3704$ & LT & $g$ & 17.95 & 0.03 & 21.00 \\
\hline ZTF19abyfazm & AT2019pwa & $2,458,737.3704$ & LT & $g$ & 17.96 & 0.01 & 21.81 \\
\hline ZTF19abyfazm & AT2019pwa & $2,458,737.3715$ & LT & $r$ & 18.30 & 0.01 & 22.36 \\
\hline ZTF19abyfazm & AT2019pwa & $2,458,737.3725$ & LT & $i$ & 18.65 & 0.05 & 21.00 \\
\hline ZTF19abyfazm & AT2019pwa & $2,458,737.3725$ & LT & $i$ & 18.62 & 0.01 & 22.16 \\
\hline
\end{tabular}

ZTF19abyfazm/AT2019pvz-Among the other candidates identified in Anand et al. (2019), we highlighted this one as being blue $(g-r \sim 0.4)$, with its last nondetection 1 day before the merger, and a faint source in PS1 about 2". 5 from the transient position. Our imaging and spectroscopy with the LT showed that the transient remained bright and blue, with no obvious emission or absorption lines in the spectrum, suggesting that this was likely a CV (Perley \& Copperwheat 2019a); this conclusion was further supported by a GTC spectrum (Castro-Tirado et al. 2019b).

ZTF19abyfbii/AT2019pwa-During the same initial search, we identified ZTF19abyfbii, whose proximity to an SDSS galaxy with a photometric redshift of $z[p]=0.124$ placed it within the distance uncertainty for S190910d (Anand et al. 2019). Our candidate was classified as an SN Ia at $z$ $[s]=0.1286 \pm 0.0005$ less than $20 \mathrm{hr}$ later by GTC using the $\mathrm{H} \alpha, \mathrm{H} \beta$, and $\mathrm{O}$ II lines in its spectrum (Castro-Tirado et al. 2019b). Further spectroscopy with the William Hershel Telescope provided a detailed classification that this transient was SN Ia 91T-like, 5 days before the peak, at $z[s]=0.118$ (Cannizzaro et al. 2019).

\section{B.6. $S 190910 h$}

We summarize the candidate counterparts to S190910h in Table 5 and the follow-up photometry in Table 14. Next, we discuss why we conclude that each one is unrelated.

\section{B.6.1. Spectroscopically Classified}

ZTF19abyheza/AT2019pxi-We initially detected ZTF19abyheza at $g=19.14 \pm 0.13$ with the ZTF with heavy galactic extinction of $\sim 0.8$ in the direction of the transient. One day later, Valeev et al. (2019) imaged the transient, reporting that it had brightened to $r=18.74 \pm 0.05$. The GTC spectroscopy revealed $\mathrm{H} \alpha$ in emission and $\mathrm{H} \beta$ in absorption at $z[s]=0$. Synthesizing this information along with the light-curve shape suggested that this was likely a CV.

ZTF19abyhhml/AT2019pxj-According to our machinelearning algorithms derived from the PS1-DR2 catalog, we could not clearly determine whether this source was of stellar origin. Similar to the previous transient, GTC imaging demonstrated that the light curve had risen to $r=19.26 \pm 0.04$, and spectra exhibited the He II and He I lines and a double-peaked $\mathrm{H} \alpha$ line, confirming that it was also a galactic $\mathrm{CV}$.

ZTF19abyirjl/AT2019pxe-We highlighted ZTF19abyirjl as being of interest due to its photometric redshift, $0.1 \pm 0.017$. Having no other information about the transient, we monitored the light curve for several days and determined it was too slow to be associated with the GW event, with an average flat evolution. One month later, we obtained a spectrum using P200 + DBSP, which clearly demonstrated through Si II lines that it was an SN Ia.

ZTF19abygvmp/AT2019pzg-This candidate was among those candidates reported in our second set of transients (Stein et al. 2019a). We highlighted ZTF19abygvmp, a transient detected $1 \mathrm{hr}$ after the merger time, in a slightly offset position from the galaxy, as it had appeared to have risen by $0.5 \mathrm{mag}$ since the last nondetection. Cannizzaro et al. acquired a WHT spectrum of the source about 2 days later, but the spectrum, dominated by host galaxy light, yielded only a redshift of $z$ $[s]=0.049$, exactly consistent with the LVC distance estimate. Two weeks later, we obtained an LRIS spectrum of the source, classifying it as an SN II (also consistent with its slow photometric evolution).

\section{B.6.2. Slow Photometric Evolution}

ZTF19abylleu/AT2019pyu-23 hr after the merger, we detected this bright $(r=19.25 \mathrm{mag})$ transient with an upper limit of $r=20.4$ mag from the day before. Though we could not obtain any spectra, we continued tracking the evolution of the transient over a period of $\sim 25$ days; the $r$-band light curve remained relatively flat, while the $g$-band light curve exhibited a gradual decline. We concluded that the evolution was too slow $\left(\alpha_{g}=0.03\right)$ to be associated with the $\mathrm{GW}$ event.

ZTF19abyjiw-Valeev et al. (2019) obtained a spectrum with GTC about 2 days later that appeared to be a featureless blue continuum from which they could not derive a conclusive 
Table 14

Follow-up Photometry for S190910h Candidates

\begin{tabular}{|c|c|c|c|c|c|c|c|}
\hline Name & IAU Name & Date & Telescope & Filter & $m(\mathrm{AB})$ & $\sigma_{m}$ & $m_{\lim }$ \\
\hline ZTF19abyjcom & AT2019pxk & $2,458,737.5558$ & LT & $g$ & 99.0 & 99.0 & 20.75 \\
\hline ZTF19abyjcom & AT2019pxk & $2,458,737.5569$ & LT & $r$ & 99.0 & 99.0 & 20.71 \\
\hline ZTF19abyjcom & AT2019pxk & $2,458,737.5579$ & LT & $i$ & 99.0 & 99.0 & 20.21 \\
\hline ZTF19abyjcon & AT2019pxl & $2,458,737.6142$ & LT & $g$ & 99.0 & 99.0 & 21.29 \\
\hline ZTF19abyjcon & AT2019pxl & $2,458,737.6152$ & LT & $r$ & 99.0 & 99.0 & 21.44 \\
\hline ZTF19abyjcon & AT2019pxl & $2,458,737.6163$ & LT & $i$ & 99.0 & 99.0 & 21.33 \\
\hline ZTF19abyjcoo & AT2019pxm & $2,458,737.6234$ & LT & $g$ & 99.0 & 99.0 & 20.84 \\
\hline ZTF19abyjcoo & AT2019pxm & $2,458,737.6245$ & LT & $r$ & 99.0 & 99.0 & 20.89 \\
\hline ZTF19abyjcoo & AT2019pxm & $2,458,737.6255$ & LT & $i$ & 99.0 & 99.0 & 21.30 \\
\hline
\end{tabular}

Table 15

Follow-up Photometry for S190930t Candidates

\begin{tabular}{|c|c|c|c|c|c|c|c|}
\hline Name & IAU Name & Date & Telescope & Filter & $m(\mathrm{AB})$ & $\sigma_{m}$ & $m_{\mathrm{lim}}$ \\
\hline ATLAS19wyn & AT2019rpj & $2,458,758.0974$ & LOT & $g$ & 19.65 & 0.08 & 99.0 \\
\hline ATLAS19wyn & AT2019rpj & $2,458,758.0974$ & LOT & $r$ & 19.58 & 0.09 & 99.0 \\
\hline ATLAS19wyn & AT2019rpj & $2,458,758.0974$ & LOT & $i$ & 19.55 & 0.12 & 99.0 \\
\hline ATLAS19wyn & AT2019rpj & $2,458,758.8562$ & LDT & $r$ & 19.6 & 0.1 & 22.8 \\
\hline ZTF19acbpqlh & AT2019rpn & $2,458,758.0937$ & LOT & $g$ & 20.80 & 0.25 & 99.0 \\
\hline ZTF19acbpqlh & AT2019rpn & $2,458,758.0937$ & LOT & $r$ & 20.67 & 0.33 & 99.0 \\
\hline ZTF19acbpqlh & AT2019rpn & $2,458,758.0937$ & LOT & $i$ & 20.80 & 0.39 & 99.0 \\
\hline ZTF19acbpqlh & AT2019rpn & $2,458,758.8548$ & LDT & $r$ & 19.80 & 0.10 & 22.8 \\
\hline
\end{tabular}

classification. However, the transient presents a flat evolution, with a coefficient $\alpha<0.1$. Another detection by the ZTF (4 months after merger) suggests that it could be a CV.

ZTF19abyiwiw/AT2019pzi-We identified this transient in spatial and temporal coincidence with both S190910d and $\mathrm{S} 190910 \mathrm{~h}$ at $3^{\circ} .1$ galactic latitude and $2.3 \mathrm{mag}$ of extinction in the direction of the transient. It was first discovered at $r=20.16 \mathrm{mag}$, but photometric follow-up determined that its evolution was too slow to be relevant, with $\alpha_{g}=0.20$.

ZTF19abymhyi/AT2019pzh-The object ZTF19abymhyi was faint and hostless, with detections in the $g$ band $2 \mathrm{hr}$ after the merger (Stein et al. 2019a) and upper limits of $g=20.65 \mathrm{mag}$ from the day before. The transient rose by $\sim 0.3$ mag 1 day later. However, it was ruled out because its photometric evolution did not pass our threshold, as it faded slower than expected with $\alpha_{g}=0.03$.

ZTF19abyjcoo/AT2019pxm-This orphan transient was discovered at $r=20.28 \mathrm{mag}$, and we rule it out due to its slow evolution $\left(\alpha_{r}=0.06\right)$.

\section{B.6.3. Artifacts}

ZTF19abyjcom/AT2019pxk, ZTF19abyjcon/AT2019pxl-On the first night of observations following this GW event, we detected two hostless transients within the same exposure, detected within the same sky region. Imaging with the LT about 1 day later resulted in nondetections of both transients, despite the fact that other transients of a similar magnitude, discovered within the same exposure, were detected. Furthermore, despite clear detections initially in the $r$ and $g$ bands, we could not detect these transients in future serendipitous observations of the sky region with the ZTF. We posit that these three transients are likely cross-talk artifacts that occurred within the same exposure and therefore are unrelated.

\section{B.7. $S 190923 y$}

We summarize one candidate counterpart to S190923y in Table 6. Despite the small sky localization, the position of S190923y on the sky made it particularly challenging to access. For that reason, we chose to conduct a fully serendipitous search in ZTF data.

ZTF19acbmopl/AT2019rob-We found this transient with a photometric redshift of $\lesssim 0.03$, consistent with the LVC distance reported, slightly off the nucleus of its host galaxy. It showed a slow evolution in both the $r$ and $g$ bands: $\alpha_{r}=0.03$ and $\alpha_{g}=0.03$.

\section{B.8. $S 190930 t$}

We summarize the candidate counterparts to S190930t in Table 7 and the follow-up photometry in Table 15. Next, we discuss why we conclude that each one is unrelated.

\section{B.8.1. Spectroscopically Classified}

ZTF19acbpqlh/AT2019rpn-We first detected this candidate $13.4 \mathrm{hr}$ after the merger using our AMPEL pipeline with a magnitude of $g=20.36 \mathrm{mag}$ and upper limits of $g=20.77$ mag from 3 days before the merger. The transient was at a galactic latitude of $b=-8.49$. Using its spectroscopic host galaxy redshift, $z[s]=0.026$, we derived an absolute magnitude of $-14.91 \mathrm{mag}$ (Stein et al. 2019b). The same night, we obtained a spectrum with P200+DBSP revealing a mostly featureless blue continuum with a weak broad feature around $\mathrm{H} \alpha$ suggesting that the transient could be a young core-collapse SN. Using the ZTSh $2.6 \mathrm{~m}$ telescope at the CrAO observatory, Mazaeva et al. (2019) imaged the SN and found that its $B-R$ color of 0.5 mag was unlike what was expected of any optical transient associated with a GW event. 
Table 16

Follow-up Photometry for S191205ah Candidates

\begin{tabular}{|c|c|c|c|c|c|c|c|}
\hline Name & IAU Name & Date & Telescope & Filter & $m(\mathrm{AB})$ & $\sigma_{m}$ & $m_{\mathrm{lim}}$ \\
\hline ZTF19acxowrr & AT2019wib & $2,458,850.0554$ & P60 & $r$ & 18.91 & 0.16 & 99.0 \\
\hline ZTF19acxowrr & AT2019wib & $2,458,852.7504$ & $\mathrm{P} 60$ & $i$ & 99.0 & 99.0 & 20.00 \\
\hline ZTF19acyitga & AT2019wmn & $2,458,837.8427$ & P60 & $r$ & 18.21 & 0.07 & 99.0 \\
\hline
\end{tabular}

We followed up by taking a second spectrum with the DBSP on 2019 October 5 and confirmed that the candidate was indeed an SN II.

ZTF19acbwaah/AT2019rpp-22 hr after the merger, we detected this transient, whose slight offset from a potential galaxy host at $z[s]=0.032$ lends it an absolute magnitude of -18.069 (Stein et al. 2019b). The next night, we conducted observations of this candidate with the DBSP; the spectrum was consistent with an SN Ia a few weeks after maximum light located at $z[s]=0.03$ (Karambelkar et al. 2019b).

ATLAS19wyn/AT2019rpj — With the ZTF, we independently detected a candidate first reported by ATLAS (Smartt et al. 2019; ZTF19acbpsuf) $13.8 \mathrm{hr}$ after the merger; ATLAS detected it $4 \mathrm{hr}$ later. The transient had a deep upper limit of 20.92 from about 6 days before the merger, and its association with a host at $z[s]=0.0297$ translated to an absolute magnitude of -15.987 . The strong Balmer P Cygni features in our DBSP spectrum, taken the same night as the initial detection, clearly indicated that the transient was an SN (Karambelkar et al. 2019a).

\section{B.9. S191205ah}

We summarize the candidate counterparts to S191205ah in Table 8 and follow-up photometry in Table 16. Next, we discuss why we conclude that each one is unrelated.

\section{B.9.1. Spectroscopically Classified}

ZTF19acyifj/AT2019wmy-This transient was discovered at $r=20.09 \mathrm{mag}$ and observed by GTC at a magnitude of $r=19.79$ mag hours after the trigger. A faint host is visible in the PS1 images of the field. However, the GTC spectrum showed an SN Ia at a redshift of $z[s]=0.081$ (Hu et al. 2019a).

ZTF19acxowrr/AT2019wib-The first detection of this transient was $\sim 4$ days after the $\mathrm{GW}$ event at $r=19.054 \pm 0.13 \mathrm{mag}$. It rose over the first $\sim 15$ days, during which several spectra were taken. The first classification came from GTC (Hu et al. 2019c): an SN II at a redshift of $z[s]=0.05$.

ZTF19acyitga/AT2019wmn-This transient was located in a galaxy at a redshift of $z[s]=0.071$ and first detected at $r=19.26$ mag. We obtained an LT spectrum of ZTF19acyitga 14 days after the discovery that showed it was an SN Ia.

\section{B.9.2. Slow Photometric Evolution}

ZTF19acxpnvd/AT2019wkv-This transient was reported in Andreoni et al. (2019b) after its discovery at $r=19.4 \mathrm{mag}$. The transient was located in the outskirts of a galaxy located at a photometric SDSS redshift of $z[p] \lesssim 0.03$, and it was ruled out due to the slow evolution shown after peaking, with $\alpha_{g}=0.06$.

ZTF19acxoywk/AT2019wix-Similarly, this transient was reported in Andreoni et al. (2019b) with a discovery magnitude of $r=19.75 \mathrm{mag}$. It was located in the outer regions of a galaxy with a spectroscopic redshift of $z[s]=0.05$; however, the evolution of this transient was only $\alpha_{g}=-0.15$.

ZTF19acxoyra/AT2019wid-This slow-evolving transient was highlighted in Andreoni et al. (2019b) after being discovered at $r=19.20 \mathrm{mag}$ in the nucleus of a galaxy at $z$ $[s]=0.09$. However, it had an almost flat evolution after reaching its peak $\left(\alpha_{g}=0.05\right)$.

ZTF19acxpwlh/AT2019wiy-This transient was located in a galaxy at an SDSS photometric redshift of $z[p]=0.12$. Discovered at $g=19.84$, it showed an almost flat evolution over the days after reaching its peak $\left(\alpha_{r}=0.07\right)$.

\section{B.10. $5191213 g$}

We summarize the candidate counterparts to S191213g in Table 9 and the follow-up photometry in Table 17. Next, we discuss why we conclude that each one is unrelated.

\section{B.10.1. Spectroscopically Classified}

ZTF19acykzsk/SN2019wqj-This transient was discovered at $g=19.25 \mathrm{mag}$ in a galaxy at $z[s]=0.021$. It was not detected in the ultraviolet by the Swift telescope (Oates et al. 2019). The spectrum taken with the SPRAT on the LT (Perley \& Copperwheat 2019b) and the GMOS-N mounted on the Gemini-North $8 \mathrm{~m}$ telescope (Fremling et al. 2019) showed prominent hydrogen lines and was classified as an SN II. This was later confirmed by a GTC spectrum that showed similar features (Elias-Rosa et al. 2019). Furthermore, this transient had PS1 detections $\sim 1$ day after the event (Smith et al. 2019). Part of the evolution of this transient was followed up by the LOT (Tan et al. 2019).

ZTF19acymaru/AT2019wnh-This transient was discovered at $r=20.03 \mathrm{mag}$ and highlighted in Andreoni et al. (2019a). The ZTF reference image did not show a visible host. Finally, the GTC spectrum revealed an SN Ia at redshift $z[s]=0.167$ (Castro-Tirado et al. 2019a).

ZTF19acykzsp/AT2019wne-This candidate was first highlighted in Andreoni et al. (2019a), as it was discovered at $r=20.18 \mathrm{mag}$. The LT/SPRAT spectrum showed an SN Ia at maximum light at $z[s]=0.16$ (Perley \& Copperwheat 2019b).

ZTF19acyfoha/AT2019wkl-Similarly, ZTF19acyfoha was reported in Andreoni et al. (2019a) at $g=17.49$ mag. It was located in one of the arms of a spiral galaxy with a CLU redshift of $z[p]=0.04$. The candidate was observed with the SEDM at the P60, and its spectra showed clear features of an SN Ia at $z[s]=0.044$.

ZTF19acymcwv/AT2019wni-This transient was discovered at $r=20.24 \mathrm{mag}$ and reported in Andreoni et al. (2019a). The candidate is in the outskirts of an elliptical galaxy, and a spectrum taken with WHT revealed an SN Ia at $z[s]=0.09$ (Brennan et al. 2019).

ZTF19acymixu/AT2019wrr-This candidate was first reported in Stein et al. (2019d), as it was discovered at $r=19.87 \mathrm{mag}$ on top of a faint diffuse source. After 
Table 17

Follow-up Photometry for S191213g Candidates

\begin{tabular}{|c|c|c|c|c|c|c|c|}
\hline Name & IAU Name & Date & Telescope & Filter & $m(\mathrm{AB})$ & $\sigma_{m}$ & $m_{\mathrm{lim}}$ \\
\hline ZTF19acykzsk & SN2019wqj & $2,458,831.8323$ & $\mathrm{P} 60$ & $r$ & 19.06 & 0.08 & 20.34 \\
\hline ZTF19acykzsk & SN2019wqj & $2,458,831.928$ & LOT & $g$ & 19.37 & 0.10 & 99.0 \\
\hline ZTF19acykzsk & SN2019wqj & $2,458,831.931$ & LOT & $r$ & 19.11 & 0.16 & 99.0 \\
\hline ZTF19acykzsk & SN2019wqj & $2,458,831.935$ & LOT & $i$ & 19.10 & 0.11 & 99.0 \\
\hline ZTF19acykzsk & SN2019wqj & $2,458,832.223$ & LOT & $g$ & 19.51 & 0.11 & 99.0 \\
\hline ZTF19acykzsk & SN2019wqj & $2,458,832.231$ & LOT & $r$ & 19.10 & 0.14 & 99.0 \\
\hline ZTF19acykzsk & SN2019wqj & $2,458,832.233$ & LOT & $i$ & 19.06 & 0.24 & 99.0 \\
\hline ZTF19acykzsk & SN2019wqj & $2,458,832.2910$ & UVOT & $v$ & 99.0 & 99.0 & 17.2 \\
\hline ZTF19acykzsk & SN2019wqj & $2,458,832.2910$ & UVOT & $b$ & 99.0 & 99.0 & 17.8 \\
\hline ZTF19acykzsk & SN2019wqj & $2,458,832.2910$ & UVOT & $u$ & 99.0 & 99.0 & 17.5 \\
\hline ZTF19acykzsk & SN2019wqj & $2,458,832.2910$ & UVOT & $w 1$ & 99.0 & 99.0 & 17.5 \\
\hline ZTF19acykzsk & SN2019wqj & $2,458,832.2910$ & UVOT & $m 2$ & 99.0 & 99.0 & 18.0 \\
\hline ZTF19acykzsk & SN2019wqj & $2,458,832.2910$ & UVOT & $w 2$ & 99.0 & 99.0 & 18.1 \\
\hline ZTF19acymixu & AT2019wrr & $2,458,832.2910$ & UVOT & $v$ & 99.0 & 99.0 & 19.5 \\
\hline ZTF19acymixu & AT2019wrr & $2,458,832.2910$ & UVOT & $b$ & 20.10 & 0.4 & 99.0 \\
\hline ZTF19acymixu & AT2019wrr & $2,458,832.2910$ & UVOT & $u$ & 99.0 & 99.0 & 19.7 \\
\hline ZTF19acymixu & AT2019wrr & $2,458,832.2910$ & UVOT & $w 1$ & 99.0 & 99.0 & 19.7 \\
\hline ZTF19acymixu & AT2019wrr & $2,458,832.2910$ & UVOT & $m 2$ & 99.0 & 99.0 & 19.7 \\
\hline ZTF19acymixu & AT2019wrr & $2,458,832.2910$ & UVOT & $w 2$ & 99.0 & 99.0 & 20.3 \\
\hline ZTF19acymaru & AT2019wnh & $2,458,831.9682$ & LCOGT1m & $g$ & 19.83 & 0.04 & 21.00 \\
\hline ZTF19acymaru & AT2019wnh & $2,458,831.9706$ & LCOGT1m & $i$ & 20.23 & 0.15 & 21.00 \\
\hline ZTF19acymaru & AT2019wnh & $2,458,831.9755$ & LCOGT1m & $r$ & 20.11 & 0.05 & 21.00 \\
\hline ZTF19acyfoha & AT2019wkl & $2,458,831.7544$ & $\mathrm{P} 60$ & $r$ & 17.29 & 0.05 & 19.19 \\
\hline ZTF19acyldun & AT2019wrt & $2,458,853.7823$ & P60 & $i$ & 18.99 & 0.10 & 19.87 \\
\hline ZTF19acyldun & AT2019wrt & $2,458,832.2910$ & UVOT & $v$ & 99.0 & 99.0 & 17.9 \\
\hline ZTF19acyldun & AT2019wrt & $2,458,832.2910$ & UVOT & $b$ & 18.83 & 0.13 & 99.0 \\
\hline ZTF19acyldun & AT2019wrt & $2,458,832.2910$ & UVOT & $u$ & 18.18 & 0.12 & 99.0 \\
\hline ZTF19acyldun & AT2019wrt & $2,458,832.2910$ & UVOT & $w 1$ & 17.62 & 0.11 & 99.0 \\
\hline ZTF19acyldun & AT2019wrt & $2,458,832.2910$ & UVOT & $m 2$ & 17.71 & 0.13 & 99.0 \\
\hline ZTF19acyldun & AT2019wrt & $2,458,832.2910$ & UVOT & $w 2$ & 18.19 & 0.12 & 99.0 \\
\hline
\end{tabular}

$\sim 1.6$ days, observations with Swift showed a source at $b=20.1$ mag. However, it was later classified as an SN Ia at $z[s]=0.14$ with a spectrum taken with the DBSP at the P200.

ZTF19acylvus/AT2019wnk-This transient was discovered at $r=19.60 \mathrm{mag}$, sitting on top of a faint galaxy without a known redshift. It was classified by the GTC as an SN Ia at $z$ $[s]=0.1$ (Castro-Tirado et al. 2019a).

ZTF19acymcna/AT2019wnn-This transient was detected at $r=20.74 \mathrm{mag}$ in the nucleus of an elliptical galaxy. The GTC spectrum showed broad hydrogen features at $z=0.2$, consistent with an AGN.

ZTF19acyldun/AT2019wrt-This candidate was reported with an initial magnitude of $g=19.8$. The follow-up with the Swift telescope shown an active source in the ultraviolet (Oates et al. 2019). The observations performed by GTC discovered a source at $z[s]=0.057$ with narrow Balmer lines consistent with a luminous blue variable (LBV; Castro-Tirado et al. 2019a), as it was also detected in 2012 by PS1. However, the source brightened to a peak absolute magnitude of $\approx-18 \mathrm{mag}$, and we revise its classification to be an SN IIn. It additionally faded at a rate much slower than our $\alpha=0.3$ mag evolution threshold, with a coefficient of $\alpha_{r}=0.09$.

\section{B.10.2. Slow Photometric Evolution}

ZTF19acykyzj/AT2019wrg-This candidate was discovered at $g=20.55$ and reported in Stein et al. (2019d). It was located in the outskirts of a spiral galaxy at unknown redshift; however, its slow magnitude evolution $\left(\alpha_{r}=-0.03\right)$ makes this transient not relevant.

ZTF19acymapa/AT2019wro-This source was detected at $g=20.31$ and reported in Stein et al. (2019d). To calculate the evolution of this object, we have only used the first 2 nights of data, as there are no more data on this transient. Using this $\Delta t$, we obtain a slow-evolving transient with $\alpha_{r}=-0.06$. Additionally, we note that the first two data points make a color consistent with $g-r=0$.

ZTF19acymaxu/AT2019wrp-This candidate was highlighted in Stein et al. (2019d) at $r=18.70 \mathrm{mag}$. It is on top of a faint PS1 source, and its slow magnitude evolution of $\alpha_{r}=0.03$ allows us to rule it out.

ZTF19acymlhi/AT2019wrs-The first detection of this candidate was of $r=19.54 \mathrm{mag}$, and its initial color was consistent with $g-r=0$ mag. Similar to ZTF19acymapa, the baseline used in this case was of $\Delta t=2$ days, and the evolution showed a slow rise of $\alpha_{r}=-0.17$.

\section{B.10.3. Artifacts}

ZTF19acykwsd/AT2019wnl-This transient was highlighted as an orphan source with two detections in different bands: $r=19.42$ and $g=19.39 \mathrm{mag}$. We proceeded to obtain an LT/ SPRAT spectrum; however, the source was not present in the acquisition image. Further investigation showed more sources around ZTF19acykwsd consistent with cross-talk. 


\section{B.10.4. Stellar Sources}

ZTF19acykyqu/AT2019wre-This transient was detected at $g=21.13 \mathrm{mag}$ and has a second detection $3.5 \mathrm{hr}$ later at $r=20.86$ mag. There are no more ZTF data on this object; however, there is a faint point source underneath the transient and a PS1-DR2 detection $\sim 1$ month before the GW event. We then consider ZTF19acykyqu to be related to a stellar background source.

ZTF19acykyrz/AT2019wrf - Similar to ZTF19acykyqu, this source sits on a PS1 source that has a previous variability history. The first PS1-reported detection was in 2010, while the last PS1-reported detection was in 2014. As the ZTF only detected this source twice, at $g=20.97$ and $r=20.16 \mathrm{mag}$, we posit that this candidate is related to the PS1 source underneath.

ZTF19acykzfy/AT2019wrh-This orphan transient was first discovered at $g=20.56$ and detected $\sim 3.5 \mathrm{hr}$ later at $r=$ $20.96 \mathrm{mag}$. The galactic latitude of ZTF19acykzfy $(b=$ $\left.-15^{\circ} .73\right)$ and a nearby $\left(<3^{\prime \prime}\right)$ detection in the PS1-DR2 catalog back the stellar origin of this transient.

ZTF19acyldum/AT2019wrn-The candidate was first reported by Stein et al. (2019d) with a magnitude of $g=19.78$ mag. It was later detected twice: $3 \mathrm{hr}$ later at $r=19.82 \mathrm{mag}$ and $5 \mathrm{hr}$ later at $g=19.84 \mathrm{mag}$. However, there is a PS1-DR2 detection within $1^{\prime \prime}$ in 2010 and a faint source in the ZTF reference images. Therefore, we posit this candidate as a stellar variable and thus unrelated.

\section{B.11. S200105ae and S200115j}

For candidates identified within the sky map of S200105ae and S200115j, see Anand et al. (2020).

\section{B.12. $S 200213 t$}

We summarize the candidate counterparts to S200213t in Table 10 and the follow-up photometry in Table 18. Next, we discuss why we conclude that each one is unrelated. All of the transients described for this event (S200213t) were reported in Kasliwal et al. (2020).

\section{B.12.1. Spectroscopically Classified}

ZTF20aamvqxl/AT2020ciy-This transient was first reported in Kasliwal et al. (2020), as it was discovered at $g=20.45 \mathrm{mag}$, in the outskirts of a potential host. With the spectra taken with GTC, Valeev et al. (2020) classified the candidate as an SN Ia at $z[s]=0.1$.

ZTF20aamvnth/AT2020cjb-Similarly, this candidate was first reported in Kasliwal et al. (2020); however, its potential host was a faint and diffuse galaxy visible in the PS1 image of the field. A spectrum from GTC classified this candidate as an SN II at $z[s]=0.061$ (Castro-Tirado et al. 2020).

ZTF20aamvoxx/AT2020cjg-This transient was first observed at $g=19.99 \mathrm{mag}$, close to the nucleus of an elliptical galaxy. Data taken with GTC classified this candidate as an SN Ia at $z[s]=0.097$ (Valeev et al. 2020).

ZTF20aamvtip/AT2020cje-The first detection of ZTF20aamvtip was at $g=20.7 \mathrm{mag}$ and faded $0.2 \mathrm{mag}$ in the $r$ band after a day. The SDSS photometric redshift of the faint host was $z[p]=0.225$. The GTC spectra classified it as an SN Ia at $z[s]=0.15$ (Valeev et al. 2020).
ZTF20aamvnat/AT2020ciz-This transient was discovered at $g=18.93 \mathrm{mag}$, and, while originally thought orphan, a faint red counterpart in the PS1 and ZTF reference image suggested a stellar origin. Additionally, it is located at $b=-5^{\circ} .62$, backing up the stellar hypothesis. Finally, GTC spectra showed strong hydrogen lines at $z[s]=0$, consistent with a galactic $\mathrm{CV}$ (Castro-Tirado et al. 2020).

ZTF20aamvodd/AT2020cjf - Similarly, this transient sits at $b=-9^{\circ} .53$ and has a faint red PS1 counterpart. It was later classified as a stellar flare at $z[s]=0.0$ (Castro-Tirado et al. 2020) due to its $\mathrm{H} \alpha$ features.

ZTF20aamvoeh/AT2020cjc-This transient was discovered at $g=20.56$ mag on top of an elliptical galaxy. We classified the candidate as an SN Ia at $z[s]=0.14$ using the spectrum taken with the DBSP at the P200 telescope.

ZTF20aanaltd/AT2020clt-This transient was first reported in Andreoni et al. (2020c), as it was discovered at $g=20.81 \mathrm{mag}$ in the outskirts of a faint red galaxy. The spectrum from the LRIS at the Keck observatory revealed an SN Ia at $z[s]=0.2$ (De 2020).

ZTF20aanaoyz/AT2020clw-This transient was discovered at $g=21.50$ mag on top of a faint PS1 elongated source. It was classified by GTC as an SN Ia at redshift $z[s]=0.276(\mathrm{Hu}$ et al. 2020).

ZTF20aamvpvx/AT2020clx-The first observation of this transient was at $g=20.30 \mathrm{mag}$ in the nucleus of an elliptical galaxy. The GTC spectrum showed an SN II at redshift $z$ $[s]=0.074$ with prominent hydrogen features (De 2020).

ZTF20aanakcd/AT2020cmr-This candidate was discovered in the outskirts of an elongated, bright elliptical galaxy at $g=20.70$ mag. The spectrum taken with the DBSP at the P200 classified it as an SN IIn at $z[s]=0.077$ (Andreoni et al. 2020a).

ZTF20aanamcs/AT2020crc-This object was discovered close to the nucleus of an edge-on galaxy at $g=21.25 \mathrm{mag}$ and $z[s]=0.093$ and subsequently classified as an SN II (De 2020).

ZTF20aanakge/AT2020crd-This candidate was detected as an orphan at $g=20.64$ mag. The spectrum taken with OSIRIS at the GTC classified it as an SN Ia at $z[s]=0.1272$ (Hu et al. 2020).

\section{B.12.2. Stellar}

ZTF20aanaksk/AT2020clu-This candidate was first reported at $g=20.48 \mathrm{mag}$ as an orphan transient. We rule out ZTF20aanaksk, as it has two previous detections in 2010 in the PS1-DR2 catalog, and we posit that it is related to a faint star in the background.

ZTF2Oaanakes/AT2020cly_This candidate was first detected at $g=21.11 \mathrm{mag}$ and with a color consistent with $g-r=0$. Follow-up with ARCTIC and GTC left only upper limits for this fast transient (Bellm \& Graham 2020; Hu et al. 2020). However, there is an archival detection in the PS1-DR2 catalog 1."5 from the ZTF source. Thus, we reject this candidate.

\section{B.12.3. Slow Photometric Evolution}

ZTF20aamvmzj/AT2020cja-This transient sits at $b=-10^{\circ} .43$; however, it does not seem to have a PS1 or ZTF counterpart, as with the previous stellar sources. The spectra taken with Keck I+LRIS and P200 only showed a 
Table 18

Follow-up Photometry for S200213t Candidates

\begin{tabular}{|c|c|c|c|c|c|c|c|}
\hline Name & IAU Name & Date & Telescope & Filter & $m(\mathrm{AB})$ & $\sigma_{m}$ & $m_{\lim }$ \\
\hline ZTF20aamvqxl & AT2020ciy & $2,458,893.3371$ & LT & $i$ & 20.17 & 0.15 & 21.61 \\
\hline ZTF20aamvqxl & AT2020ciy & $2,458,893.3406$ & LT & $g$ & 99.0 & 99.0 & 19.54 \\
\hline ZTF20aamvoxx & AT2020cjg & $2,458,893.3733$ & LT & $i$ & 20.29 & 0.21 & 21.30 \\
\hline ZTF20aamvoxx & AT2020cjg & $2,458,893.3751$ & LT & $r$ & 21.47 & 0.19 & 22.49 \\
\hline ZTF20aamvoxx & AT2020cjg & $2,458,893.3768$ & LT & $g$ & 20.26 & 0.03 & 23.36 \\
\hline ZTF20aamvtip & AT2020cje & $2,458,893.3457$ & LT & $i$ & 20.68 & 0.07 & 22.73 \\
\hline ZTF20aamvtip & AT2020cje & $2,458,893.3475$ & LT & $r$ & 20.73 & 0.11 & 22.52 \\
\hline ZTF20aamvtip & AT2020cje & $2,458,893.3493$ & $\mathrm{LT}$ & $g$ & 20.80 & 0.06 & 23.14 \\
\hline ZTF20aamvmzj & AT2020cja & $2,458,893.3559$ & LT & $g$ & 20.45 & 0.05 & 23.10 \\
\hline ZTF20aamvmzj & AT2020cja & $2,458,906.7200$ & $\mathrm{LCO} 2 \mathrm{~m}$ & $g$ & 20.79 & 0.09 & 20.91 \\
\hline ZTF20aamvmzj & AT2020cja & $2,458,906.7350$ & LCO2m & $r$ & 20.32 & 0.09 & 21.30 \\
\hline ZTF20aamvmzj & AT2020cja & $2,458,893.9607$ & LOT & $g$ & 20.37 & 0.10 & 99.0 \\
\hline ZTF20aamvmzj & AT2020cja & $2,458,893.9607$ & LOT & $r$ & 20.58 & 0.14 & 99.0 \\
\hline ZTF20aamvmzj & AT2020cja & $2,458,893.9607$ & LOT & $i$ & 21.02 & 0.51 & 99.0 \\
\hline ZTF20aamvoeh & AT2020cjc & $2,458,893.3559$ & LT & $g$ & 20.45 & 0.05 & 23.10 \\
\hline ZTF20aamvoeh & AT2020cjc & $2,458,906.7200$ & $\mathrm{LCO} 2 \mathrm{~m}$ & $g$ & 20.79 & 0.09 & 20.91 \\
\hline ZTF20aamvoeh & AT2020cjc & $2,458,906.7350$ & $\mathrm{LCO} 2 \mathrm{~m}$ & $r$ & 20.32 & 0.09 & 21.30 \\
\hline ZTF20aanakwb & AT2020cls & $2,458,893.9607$ & LOT & $g$ & 99.0 & 99.0 & 18.9 \\
\hline ZTF20aanakwb & AT2020cls & $2,458,893.9607$ & LOT & $r$ & 21.12 & 0.32 & 99.0 \\
\hline ZTF20aanakwb & AT2020cls & $2,458,893.9607$ & LOT & $i$ & 20.97 & 0.37 & 99.0 \\
\hline ZTF20aanaltd & AT2020clt & $2,458,893.9607$ & LOT & $g$ & 21.47 & 0.24 & 99.0 \\
\hline ZTF20aanaltd & AT2020clt & $2,458,893.9607$ & LOT & $r$ & 19.34 & 0.04 & 99.0 \\
\hline ZTF20aanaltd & AT2020clt & $2,458,893.9607$ & LOT & $i$ & 19.98 & 0.12 & 99.0 \\
\hline ZTF20aanaksk & AT2020clu & $2,458,893.9607$ & LOT & $g$ & 20.80 & 0.14 & 99.0 \\
\hline ZTF20aanaksk & AT2020clu & $2,458,893.9607$ & LOT & $r$ & 20.79 & 0.15 & 99.0 \\
\hline ZTF20aanaksk & AT2020clu & $2,458,893.9607$ & LOT & $i$ & 21.19 & 0.47 & 99.0 \\
\hline ZTF20aanaoyz & AT2020clw & $2,458,893.9607$ & LOT & $g$ & 21.46 & 0.42 & 99.0 \\
\hline ZTF20aanaoyz & AT2020clw & $2,458,893.9607$ & LOT & $r$ & 21.09 & 0.22 & 99.0 \\
\hline ZTF20aanaoyz & AT2020clw & $2,458,893.9607$ & LOT & $i$ & 20.75 & 0.37 & 99.0 \\
\hline ZTF20aanakes & AT2020cly & $2,458,894.5992$ & APO & $g$ & 99.0 & 99.0 & 23.50 \\
\hline ZTF20aanakes & AT2020cly & $2,458,894.6012$ & $\mathrm{APO}$ & $i$ & 99.0 & 99.0 & 21.50 \\
\hline ZTF20aanakes & AT2020cly & $2,458,894.6031$ & APO & $r$ & 99.0 & 99.0 & 23.00 \\
\hline
\end{tabular}

featureless blue continuum (De 2020). It was first observed (Oates et al. 2020b) by the UVOT (Roming et al. 2005) at Swift 6.7 days after the merger, and it was only detected in the $u$ band at $u=19.05$ mag. It was later followed up but not detected in any bandpass (Oates et al. 2020a). Nonetheless, the magnitude evolution of the transient was otherwise flat, and it slowly faded over time with $\alpha_{r}=0.04$.

ZTF20aanaqhe/AT2020cre-This transient was detected at $g=20.88 \mathrm{mag}$ on an elliptical galaxy at a photometric redshift of $z[p]=0.16$. Its slow rise of $\alpha_{g}=-0.08$ was inconsistent with the rise of a fast transient.

ZTF20aanakwb/AT2020cls-This transient was first reported in Andreoni et al. (2020c) at $g=21.03 \mathrm{mag}$, offset from a bright Gaia point source $(g=15.27 \mathrm{mag})$. This transient was detected by the LOT $12 \mathrm{hr}$ later at an $r$-band magnitude consistent with no evolution. The initial color $g-r$ is consistent with 0 mag. In the ZTF reference image, there is a faint point source, which indicates stellar activity.

\section{B.12.4. Outside the GW Map}

ZTF20aanallx/AT2020clv-This transient was first reported in Andreoni et al. (2020c) at $g=21.11 \mathrm{mag}$ and discovered at a galactic latitude of $b=-11^{\circ} .43$. It is offset from an elliptical galaxy; however, it falls in a fairly crowded region. The rejection criterion we used for this transient is the fact that it is not within the $95 \%$ credible level of the latest LALInference map for S200213t.

\section{ORCID iDs}

Mansi M. Kasliwal (1) https://orcid.org/0000-0002-5619-4938 Shreya Anand (1) https://orcid.org/0000-0003-3768-7515 Tomás Ahumada (1) https://orcid.org/0000-0002-2184-6430 Robert Stein (1) https://orcid.org/0000-0003-2434-0387 Igor Andreoni (1) https://orcid.org/0000-0002-8977-1498 Michael W. Coughlin (1) https://orcid.org/0000-00028262-2924

Leo P. Singer (1) https://orcid.org/0000-0001-9898-5597

Erik C. Kool 이 https://orcid.org/0000-0002-7252-3877

Yuhan Yao (i) https://orcid.org/0000-0001-6747-8509

Mattia Bulla (1) https://orcid.org/0000-0002-8255-5127

Dougal Dobie (1) https://orcid.org/0000-0003-0699-7019

Daniel A. Perley (1) https://orcid.org/0000-0001-8472-1996

S. Bradley Cenko (1) https://orcid.org/0000-0003-1673-970X

Varun Bhalerao iㅏ https://orcid.org/0000-0002-6112-7609 
David L. Kaplan (1) https://orcid.org/0000-0001-6295-2881

Jesper Sollerman (ib https://orcid.org/0000-0003-1546-6615 Ariel Goobar (ㄴ) https://orcid.org/0000-0002-4163-4996 Christopher M. Copperwheat (10 https://orcid.org/0000-00017983-8698

Eric C. Bellm (10) https://orcid.org/0000-0001-8018-5348 G. C. Anupama (ib https://orcid.org/0000-0003-3533-7183 Alessandra Corsi (1) https://orcid.org/0000-0001-8104-3536 Samaya Nissanke (1) https://orcid.org/0000-0001-6573-7773 Sudhanshu Barway (i) https://orcid.org/0000-0002-3927-5402 Joshua S. Bloom (1) https://orcid.org/0000-0002-7777-216X Kevin B. Burdge (1) https://orcid.org/0000-0002-7226-836X David O. Cook (1) https://orcid.org/0000-0002-6877-7655 Jeff Cooke (1) https://orcid.org/0000-0001-5703-2108 Virginia Cunningham (1) https://orcid.org/0000-00032292-0441

Dmitry A. Duev (1) https://orcid.org/0000-0001-5060-8733

Anna Franckowiak (1) https://orcid.org/0000-0002-5605-2219

Sara Frederick (1) https://orcid.org/0000-0001-9676-730X Christoffer Fremling (1) https://orcid.org/0000-00024223-103X

Avishay Gal-Yam (i) https://orcid.org/0000-0002-3653-5598 Pradip Gatkine (1) https://orcid.org/0000-0002-1955-2230 Shaon Ghosh (1) https://orcid.org/0000-0003-4259-8592 Daniel A. Goldstein (1) https://orcid.org/0000-0003-3461-8661 V. Zach Golkhou (10) https://orcid.org/0000-0001-8205-2506 Matthew J. Graham (1) https://orcid.org/0000-0002-3168-0139 Melissa L. Graham (i) https://orcid.org/0000-0002-9154-3136 Matthew J. Hankins (ㄷ) https://orcid.org/0000-0001-9315-8437 George Helou (1) https://orcid.org/0000-0003-3367-3415 Youdong Hu @i https://orcid.org/0000-0002-7400-4608 Amruta Jaodand (1) https://orcid.org/0000-0002-3850-6651 Albert K. H. Kong (1) https://orcid.org/0000-0002-5105-344X S. R. Kulkarni ii https://orcid.org/0000-0001-5390-8563 Brajesh Kumar 10 https://orcid.org/0000-0001-7225-2475 Russ R. Laher (1) https://orcid.org/0000-0003-2451-5482 Ashish Mahabal (i) https://orcid.org/0000-0003-2242-0244 Frank J. Masci $\odot$ https://orcid.org/0000-0002-8532-9395 Adam A. Miller (1) https://orcid.org/0000-0001-9515-478X Kunal Mooley 나 https://orcid.org/0000-0002-2557-5180 Jeffrey A. Newman (1) https://orcid.org/0000-0001-8684-2222 Chow-Choong Ngeow (i) https://orcid.org/0000-00018771-7554

Elena Pian (ib https://orcid.org/0000-0001-8646-4858 Reed Riddle (1) https://orcid.org/0000-0002-0387-370X Maayane T. Soumagnac (i) https://orcid.org/0000-00016753-1488

Anastasios Tzanidakis (i) https://orcid.org/0000-00030484-3331

Eleonora Troja (10 https://orcid.org/0000-0002-1869-7817 Sara Webb (10) https://orcid.org/0000-0003-2601-1472 Po-Chieh Yu iㅣ https://orcid.org/0000-0001-8894-0854 Bin-Bin Zhang (ㄴ) https://orcid.org/0000-0003-4111-5958 Rongpu Zhou (10 https://orcid.org/0000-0001-5381-4372

\section{References}

Abbott, B. P., Abbott, R., Abbott, T. D., et al. 2017a, ApJL, 848, L12 Abbott, B. P., Abbott, R., Abbott, T. D., et al. 2018, LRR, 21, 3 Abbott, B. P., Abbott, R., Abbott, T. D., et al. 2020a, ApJL, 892, L3 Abbott, B. P., Abbott, R., Abbott, T. D., et al. 2016, PhRvL, 116, 061102 Abbott, B. P., Abbott, R., Abbott, T. D., et al. 2017b, PhRvL, 119, 161101 Abbott, B. P., Abbott, T. D., Abraham, S., et al. 2020b, ApJL, 896, L44
Ahumada, R., Allende Prieto, C., Almeida, A., et al. 2020, ApJS, 249, 3 Allington-Smith, J., Murray, G., Content, R., et al. 2002, PASP, 114, 892 Almualla, M., Coughlin, M. W., Anand, S., et al. 2020, MNRAS, 495, 4366 Anand, S., Andreoni, I., Khandagale, M., et al. 2019, GCN, 25706, 1

Anand, S., Coughlin, M. W., Kasliwal, M. M., et al. 2020, NatAs, in press, doi:10.1038/s41550-020-1183-3

Andreoni, I., Ackley, K., Cooke, J., et al. 2017, PASA, 34, e069

Andreoni, I., Anand, S., Bellm, E., et al. 2019a, GCN, 26424, 1

Andreoni, I., Anand, S., Coughlin, M. W., et al. 2019b, GCN, 26416, 1

Andreoni, I., De, K., Kasliwal, M., Huang, Y., \& Liu, X. 2020a, GCN, 27075,1

Andreoni, I., Goldstein, D., Anand, S., et al. 2019c, ApJL, 881, L16

Andreoni, I., Goldstein, D. A., Kasliwal, M. M., et al. 2020b, ApJ, 890, 131

Andreoni, I., Kumar, H., Karambelkar, V., et al. 2020c, GCN, 27065, 1

Antier, S., Agayeva, S., AlMualla, M., et al. 2020, arXiv:2004.04277

Arcavi, I. 2018, ApJL, 855, L23

Arcavi, I., Hosseinzadeh, G., Howell, D. A., et al. 2017, Natur, 551, 64

Assef, R. J., Stern, D., Noirot, G., et al. 2018, ApJS, 234, 23

Bailer-Jones, C. A. L., Fouesneau, M., \& Andrae, R. 2019, MNRAS, 490, 5615

Becker, A. 2015, HOTPANTS: High Order Transform of PSF ANd Template Subtraction, Astrophysics Source Code Library, ascl:1504.004

Bellm, E. C., \& Graham, M. 2020, GCN, 27118, 1

Bellm, E. C., Kulkarni, S. R., Graham, M. J., et al. 2018, PASP, 131, 018002

Bellm, E. C., \& Sesar, B. 2016, pyraf-dbsp: Reduction Pipeline for the Palomar Double Beam Spectrograph, Astrophysics Source Code Library, ascl: 1602.002

Bertin, E. 2006, in ASP Conf. Series 351, Astronomical Data Analysis Software and Systems XV, ed. C. Gabriel et al. (San Francisco, CA: ASP), 112

Bertin, E. 2011, in ASP Conf. Ser. 442, Astronomical Data Analysis Software and Systems XX, ed. I. N. Evans et al. (San Francisco, CA: ASP), 435

Bertin, E., \& Arnouts, S. 2010, SExtractor: Source Extractor, Astrophysics Source Code Library, ascl:1010.064

Bertin, E., Mellier, Y., Radovich, M., et al. 2002, in ASP Conf. Ser. 281, Astronomical Data Analysis Software and Systems XI, ed. D. A. Bohlender, D. Durand, \& T. H. Handley (San Francisco, CA: ASP), 228

Blagorodnova, N., Neill, J. D., Walters, R., et al. 2018, PASP, 130, 035003

Blondin, S., \& Tonry, J. L. 2007, ApJ, 666, 1024

Brennan, S., Killestein, T., Fraser, M., et al. 2019, GCN, 26429, 1

Broekgaarden, F. S., Justham, S., de Mink, S. E., et al. 2019, MNRAS, 490, 5228

Brown, T. M., Baliber, N., Bianco, F. B., et al. 2013, PASP, 125, 1031

Buckley, D. A. H., Hearnshaw, J. B., Nordsieck, K. H., \& O’Donoghue, D. 2003, Proc. SPIE, 4834, 264

Bulla, M. 2019, MNRAS, 489, 5037

Burdge, K., Perley, D. A., \& Kasliwal, M. 2019, GCN, 25639, 1

Burgh, E. B., Nordsieck, K. H., Kobulnicky, H. A., et al. 2003, Proc. SPIE, 4841,1463

Cannizzaro, G., Pastor-Marazuela, I., Jonker, P., Maguire, K., \& Fraser, M. 2019, GCN, 25725, 1

Cao, Y., Nugent, P. E., \& Kasliwal, M. M. 2016, PASP, 128, 114502 Castro-Tirado, A., Hu, Y.-D., Valeev, A., et al. 2019a, GCN, 26492, 1

Castro-Tirado, A., Hu, Y.-D., Valeev, A., et al. 2020, GCN, 27063, 1

Castro-Tirado, A., Valeev, A., Sokolov, V., et al. 2019b, GCN, 25721, 1

Cenko, S. B., Fox, D. B., Moon, D.-S., et al. 2006, PASP, 118, 1396

Cepa, J., Aguiar, M., Castañeda, H., et al. 2005, RMxAA, 24, 1

Chambers, K. C., Magnier, E. A., Metcalfe, N., et al. 2016, arXiv:1612.05560

Chatterjee, D., Ghosh, S., Brady, P. R., et al. 2020, ApJ, 896, 54

Cook, D. O., Kasliwal, M. M., Van Sistine, A., et al. 2019, ApJ, 880, 7

Coughlin, M. W., Ahumada, T., Cenko, S. B., et al. 2019a, PASP, 131, 048001

Coughlin, M. W., Dekany, R. G., Duev, D. A., et al. 2019b, MNRAS, 485,1412

Coughlin, M. W., Kasliwal, M. M., Perley, D. A., et al. 2019c, GCN, 24283, 1 Coughlin, M. W., Ahumada, T., Anand, S., et al. 2019d, ApJL, 885, L19 Coughlin, M. W., Tao, D., Chan, M. L., et al. 2018, MNRAS, 478, 692 Coulter, D. A., Foley, R. J., Kilpatrick, C. D., et al. 2017, Sci, 358, 1556 Cowperthwaite, P. S., Berger, E., Villar, V. A., et al. 2017, ApJL, 848, L17 Crawford, S. M., Still, M., Schellart, P., et al. 2010, Proc. SPIE, 7737, 25 Cushing, M. C., Vacca, W. D., \& Rayner, J. T. 2004, PASP, 116, 362 De, K. 2020, GCN, 27140, 1

De, K., Hankins, M. J., Kasliwal, M. M., et al. 2020a, PASP, 132, 025001 De, K., Kasliwal, M. M., Ofek, E. O., et al. 2018, Sci, 362, 201

De, K., Kasliwal, M. M., Tzanidakis, A., et al. 2020b, arXiv:2004.09029 Díaz, M. C., Macri, L. M., Garcia Lambas, D., et al. 2017, ApJL, 848, L29 
Dietrich, T., Coughlin, M. W., Pang, P. T. H., et al. 2020, arXiv:2002.11355 Djorgovski, S. G., Drake, A. J., Mahabal, A. A., et al. 2011, arXiv:1102.5004 Dobie, D., Stewart, A., Murphy, T., et al. 2019, ApJL, 887, L13 Drout, M. R., Piro, A. L., Shappee, B. J., et al. 2017, Sci, 358, 1570 Duev, D. A., Mahabal, A., Masci, F. J., et al. 2019, MNRAS, 489, 3582 Elias-Rosa, N., Benetti, S., Piranomonte, S., et al. 2019, GCN, 26428, 1 Evans, P. A., Cenko, S. B., Kennea, J. A., et al. 2017, Sci, 358, 1565 Feindt, U., Nordin, J., Rigault, M., et al. 2019, JCAP, 2019, 005 Fernández, R., Foucart, F., \& Lippuner, J. 2020, MNRAS, 497, 322 Flewelling, H. 2018, AAS Meeting, 231, 436.01 Foucart, F. 2012, PhRvD, 86, 124007

Foucart, F., Hinderer, T., \& Nissanke, S. 2018, PhRvD, 98, 081501 Fremling, C., Ahumada, T., Singer, L., De, K., \& Kasliwal, M. 2019, GCN, 26427, 1

Fremling, C., Sollerman, J., Taddia, F., et al. 2016, A\&A, 593, A68 Fremling, U. C., Miller, A. A., Sharma, Y., et al. 2020, ApJ, 895, 32 Gaia Collaboration 2018, yCat, I/345

Gehrels, N., Chincarini, G., Giommi, P., et al. 2004, ApJ, 611, 1005 Gimeno, G., Roth, K., Chiboucas, K., et al. 2016, Proc. SPIE, 9908, 99082S Goldstein, A., Veres, P., Burns, E., et al. 2017, ApJL, 848, L14 Goldstein, D. A., Andreoni, I., Nugent, P. E., et al. 2019, ApJ, 881, L7 Gompertz, B., Cutter, R., Steeghs, D., et al. 2020, MNRAS, 497, 726 Graham, M. J., Ford, K. E. S., McKernan, B., et al. 2020, PhRvL, 124, 251102 Graham, M. J., Kulkarni, S. R., Bellm, E. C., et al. 2019, PASP, 131, 078001 Guevel, D., \& Hosseinzadeh, G. 2017, Dguevel/Pyzogy: Initial Release, v0.0.1, Zenodo, doi:10.5281/zenodo.1043973

Haggard, D., Nynka, M., Ruan, J. J., et al. 2017, ApJL, 848, L25

Hallinan, G., Corsi, A., Mooley, K. P., et al. 2017, Sci, 358, 1579

Hook, I. M., Jørgensen, I., Allington-Smith, J. R., et al. 2004, PASP, 116, 425

Hotokezaka, K., Kyutoku, K., Tanaka, M., et al. 2013, ApJL, 778, L16

Hu, Y., Valeev, A., Castro-Tirado, A., et al. 2019a, GCN, 26502, 1

Hu, Y.-D., Castro-Tirado, A., Valeev, A., et al. 2020, GCN, 27154, 1

Hu, Y. D., Castro-Tirado, A. J., Valeev, A. F., et al. 2019b, GCN, 24359, 1

Hu, Y.-D., Valeev, A., Castro-Tirado, A., et al. 2019c, GCN, 26422, 1

Huang, K., Urata, Y., Ip, W., et al. 2005, NCimC, 28, 731

Huehnerhoff, J., Ketzeback, W., Bradley, A., et al. 2016, Proc. SPIE, 9908, $99085 \mathrm{H}$

Izzo, L., Leloudas, G., Bruun, S., et al. 2019, GCN, 25675, 1

Jedicke, R., Bolin, B., Granvik, M., \& Beshore, E. 2016, Icar, 266, 173

Karambelkar, V., De, K., Van Roestel, J., \& Kasliwal, M. 2019a, GCN 25921,1

Karambelkar, V., De, K., van Roestel, J., \& Kasliwal, M. M. 2019b, GCN, 25931, 1

Kasen, D., Metzger, B., Barnes, J., Quataert, E., \& Ramirez-Ruiz, E. 2017 Natur, 551, 80

Kasliwal, M. M., Cannella, C., Bagdasaryan, A., et al. 2019a, PASP, 131, 038003

Kasliwal, M. M., Cenko, S. B., Singer, L. P., et al. 2016, ApJL, 824, L24

Kasliwal, M. M., Kasen, D., Lau, R. M., et al. 2019b, MNRAS, in press, doi: $10.1093 / \mathrm{mnrasl} / \mathrm{slz007}$

Kasliwal, M. M., Nakar, E., Singer, L. P., et al. 2017, Sci, 358, 1559

Kasliwal, M. M., \& Nissanke, S. 2014, ApJL, 789, L5

Kasliwal, M. M., Perley, D., Kumar, H., et al. 2020, GCN, 27051, 1

Kawaguchi, K., Kyutoku, K., Shibata, M., \& Tanaka, M. 2016, ApJ, 825, 52

Kiuchi, K., Sekiguchi, Y., Kyutoku, K., et al. 2015, PhRvD, 92, 064034

Kruckow, M. U., Tauris, T. M., Langer, N., Kramer, M., \& Izzard, R. G. 2018 , MNRAS, 481, 1908

Law, N. M., Kulkarni, S. R., Dekany, R. G., et al. 2009, PASP, 121, 1395 Levan, A. 2020, PoS, Asterics2019, 044

Ligo Scientific Collaboration, \& VIRGO Collaboration 2019a, GCN, 24237, 1 Ligo Scientific Collaboration, \& VIRGO Collaboration 2019b, GCN, 24411, 1

Lipunov, V. M., Gorbovskoy, E., Kornilov, V. G., et al. 2017, ApJL, 850, L1

Lundquist, M. J., Paterson, K., Fong, W., et al. 2019, ApJL, 881, L26

Mahabal, A., Rebbapragada, U., Walters, R., et al. 2019, PASP, 131, 038002

Margutti, R., Berger, E., Fong, W., et al. 2017, ApJL, 848, L20

Masci, F. J., Laher, R. R., Rebbapragada, U. D., et al. 2017, PASP, 129, 014002

Masci, F. J., Laher, R. R., Rusholme, B., et al. 2018, PASP, 131, 018003

Massey, P., Dunham, E., Bida, T., et al. 2013, AAS Meeting, 221, 345.02

Mazaeva, E., Pozanenko, A., Rumyantsev, V., Belkin, S., \& Volnova, A. 2019, GCN, 25943, 1

Mooley, K. P., Frail, D. A., Dobie, D., et al. 2018, ApJL, 868, L11
Moore, A. M., \& Kasliwal, M. M. 2019, NatAs, 3, 109

Muthukrishna, D., Parkinson, D., \& Tucker, B. E. 2019, ApJ, 885, 85

Nakaoka, T., Maeda, K., Yamanaka, M., et al. 2020, arXiv:2005.02992

Nakar, E. 2020, PhR, 886, 1

Naoz, S. 2016, ARA\&A, 54, 441

Nissanke, S., Kasliwal, M., \& Georgieva, A. 2013, ApJ, 767, 124

Nordin, J., Brinnel, V., van Santen, J., et al. 2019, A\&A, 631, A147

Oates, S., Klingler, N., Page, K., et al. 2020a, GCN, 27400, 1

Oates, S., Page, K., Breeveld, A., et al. 2020b, GCN, 27153, 1

Oates, S., Page, K., De Pasquale, M., et al. 2019, GCN, 26471, 1

Oke, J. B., Cohen, J. G., Carr, M., et al. 1995, PASP, 107, 375

Patterson, M. T., Bellm, E. C., Rusholme, B., et al. 2019, PASP, 131, 018001

Perley, D., \& Copperwheat, C. 2019a, GCN, 25720, 1

Perley, D., \& Copperwheat, C. 2019b, GCN, 26426, 1

Perley, D. A. 2019, PASP, 131, 084503

Perley, D. A., Goobar, A., Kasliwal, M. M., et al. 2019a, GCN, 24331, 1

Perley, D. A., Ho, A. Y. Q., \& Copperwheat, C. M. 2019b, GCN, 25643, 1

Pian, E., D’Avanzo, P., Benetti, S., et al. 2017, Natur, 551, 67

Piascik, A. S., Steele, I. A., Bates, S. D., et al. 2014, Proc. SPIE, 9147, 91478 H

Piro, A. L., \& Kollmeier, J. A. 2018, ApJ, 855, 103

Pozanenko, A. S., Barkov, M. V., Minaev, P. Y., et al. 2018, ApJL, 852, L30

Rhodes, L., Fender, R., Williams, D., et al. 2019, GCN, 24226, 1

Rigault, M., Neill, J. D., Blagorodnova, N., et al. 2019, A\&A, 627, A115

Roming, P. W. A., Kennedy, T. E., Mason, K. O., et al. 2005, SSRv, 120, 95

Rosell, M. J. B., Rostopchin, S., Zimmerman, A., et al. 2019, GCN, 25622, 1

Rosswog, S. 2005, ApJ, 634, 1202

Sagués Carracedo, A., Bulla, M., Feindt, U., \& Goobar, A. 2020, arXiv:2004. 06137

Salsamo, I., Tomasella, L., Benetti, S., D’Avanzo, P., \& Cappellaro, E. 2019a, GCN, 25618, 1

Salmaso, I., Tomasella, L., Benetti, S., et al. 2019b, GCN, 25619, 1

Schlafly, E. F., \& Finkbeiner, D. P. 2011, ApJ, 737, 103

Shappee, B. J., Prieto, J. L., Grupe, D., et al. 2014, ApJ, 788, 48

Singer, L. P., Kasliwal, M. M., Cenko, S. B., et al. 2015, ApJ, 806, 52

Singer, L. P., \& Price, L. R. 2016, PhRvD, 93, 024013

Skrutskie, M. F., Cutri, R. M., Stiening, R., et al. 2006, AJ, 131, 1163

Smartt, S., Srivastav, S., Smith, K., et al. 2019, GCN, 25922, 1

Smartt, S. J., Chen, T. W., Jerkstrand, A., et al. 2017, Natur, 551, 75

Smith, K., Smartt, S., Young, D., et al. 2019, GCN, 26430, 1

Soares-Santos, M., Holz, D. E., Annis, J., Chornock, R., \& Herner, K. 2017 ApJL, 848, L16

Soumagnac, M. T., \& Ofek, E. O. 2018, PASP, 130, 075002

Steele, I. A., Smith, R. J., Rees, P. C., et al. 2004, Proc. SPIE, 5489, 679

Stein, R., Andreoni, I., Coughlin, M., et al. 2019a, GCN, 25727, 1

Stein, R., Kasliwal, M. M., Kool, E., et al. 2019b, GCN, 25899, 1

Stein, R., Kool, E., Kumar, H., et al. 2019c, GCN, 25656, 1

Stein, R., Reusch, S., Perley, D., Andreoni, I., \& Coughlin, M. 2019d, GCN, 26437, 1

Stein, R., van Velzen, S., Kowalski, M., et al. 2020, arXiv:2005.05340

Stephan, A. P., Naoz, S., Ghez, A. M., et al. 2019, ApJ, 878, 58

Tachibana, Y., \& Miller, A. A. 2018, PASP, 130, 128001

Tan, H.-J., Kong, A., Ngeow, C.-C., \& Ip, W.-H. 2019, GCN, 26431, 1

Tauris, T. M., Langer, N., \& Podsiadlowski, P. 2015, MNRAS, 451, 2123

Tonry, J. L., Denneau, L., Heinze, A. N., et al. 2018, PASP, 130, 064505

Troja, E., Piro, L., van Eerten, H., et al. 2017, Natur, 551, 71

Utsumi, Y., Tanaka, M., Tominaga, N., et al. 2017, PASJ, 69, 101

Vacca, W. D., Cushing, M. C., \& Rayner, J. T. 2003, PASP, 115, 389

Valeev, A., Hu, Y., Castro-Tirado, A., et al. 2020, GCN, 27060, 1

Valeev, A., Hu, Y.-D., Castro-Tirado, A., et al. 2019, GCN, 25731, 1

Valenti, S., David, J. S., Yang, S., et al. 2017, ApJL, 848, L24

Veitch, J., Raymond, V., Farr, B., et al. 2015, PhRvD, 91, 042003

Waxman, E., Ofek, E. O., Kushnir, D., \& Gal-Yam, A. 2018, MNRAS, 481,3423

Wei, J., Xin, L., Antier, S., et al. 2019, GCN, 25640, 1

West, A. A., Morgan, D. P., Bochanski, J. J., et al. 2011, AJ, 141, 97

Wilson, J. C., Eikenberry, S. S., Henderson, C. P., et al. 2003, Proc. SPIE, 4841, 451

Wright, E. L., Eisenhardt, P. R. M., Mainzer, A. K., et al. 2010, AJ, 140, 1868

Yao, Y., De, K., Kasliwal, M. M., et al. 2020, arXiv:2005.12922

Yao, Y., Miller, A. A., Kulkarni, S. R., et al. 2019, ApJ, 886, 152

Zackay, B., Ofek, E. O., \& Gal-Yam, A. 2016, ApJ, 830, 27

Zhou, R., Newman, J. A., Mao, Y.-Y., et al. 2020, arXiv:2001.06018 